\title{
Sterics and Stereoelectronics in Aza-Glycine: Impact of Aza- Glycine Preorganization in Triple Helical Collagen
}

Trevor Harris and David M. Chenoweth*

Department of Chemistry, University of Pennsylvania, 231 South 34th Street, Philadelphia, Pennsylvania 19104-6323, USA 
Table S1. Collagen crystal structure sequences.............................................................. 3

Figure S1. Theoretical Ramachandran plots (1-4). Gas phase energies ............................ S4

Figure S2. Theoretical Ramachandran plot Ac-Gly- $\mathrm{NMe}_{2}$ (1) with minima..............................S5

Figure S3. Theoretical Ramachandran plot Ac-azGly-NMe 2 (2) with minima ........................... S5

Figure S4. Theoretical Ramachandran plot Ac-Gly-NHiPr (3) with minima ............................S6

Figure S5. Theoretical Ramachandran plot Ac-azGly-NHiPr (4) with minima .........................S6

Figure S6. Theoretical Ramachandran plot Ac-Gly- $\mathrm{NMe}_{2}(\mathbf{1})$ with transition states .................. S7

Figure S7. Theoretical Ramachandran plot Ac-azGly-NMe ${ }_{2}$ (2) with transition states ...............S7

Figure S8. Theoretical Ramachandran plot Ac-Gly-NHiPr (3) with transition states ................. S8

Figure S9. Theoretical Ramachandran plot Ac-azGly-NHiPr (4) with transition states.............. S8

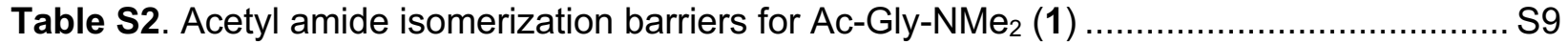

Table S3. Acetyl amide isomerization barriers for Ac-azGly-NMe $\mathrm{NM}_{2}(2) \ldots \ldots \ldots \ldots \ldots \ldots \ldots \ldots \ldots \ldots \ldots . . .59$

Table S4. Pyramidalization parameters of non-native nitrogen in model systems 2 and 4 ...... S9

Scheme S1. Isodesmic equations for 1-4. Gas phase energies ........................................ S10

Scheme S2. Isodesmic equations for 1-4. All minima................................................... $S 10$

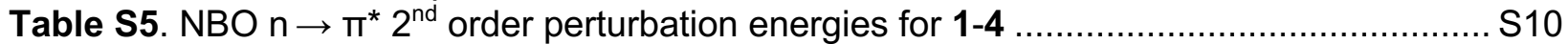

Figure S10. $360^{\circ}$ relaxed $\varphi$ dihedral scan of 2 overlaid total $n_{N} \rightarrow \sigma^{*}{ }_{N-C}$ and $n_{N} \rightarrow \sigma^{*}{ }_{N-C} \ldots \ldots . S 11$

Figure S11. Interaction analyses of triple helices using (wB97X-D) double hybrid functional. S11

Table S6. NBO charges of $\mathrm{H}$ atoms on Gly and azGly in triple helices ................................ S12

Table S7. NBO $n \rightarrow \pi^{*} 2^{\text {nd }}$ order perturbation energies in Gly triple helix ............................ S12

Table S8. NBO $n \rightarrow \pi^{*} 2^{\text {nd }}$ order perturbation energies in azGly triple helix.......................... S13

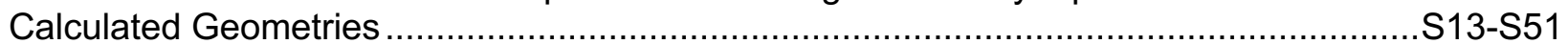

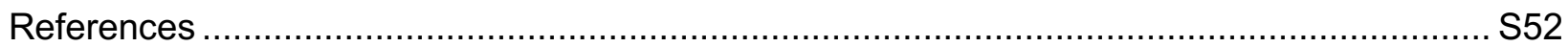




\begin{tabular}{|c|c|c|}
\hline PDB Name & Sequence & Resolution \\
\hline $3 \mathrm{AH}^{1}$ & $(\mathrm{PPG})_{9}$ & 1.08 \\
\hline $1 \mathrm{~K}^{2} \mathrm{~F}^{2}$ & $(\mathrm{PPG})_{10}$ & 1.3 \\
\hline $3 \mathrm{~B}^{2} \mathrm{~S}^{3}$ & $(\mathrm{POG})_{9}$ & 1.45 \\
\hline $1 \mathrm{~V} 7 \mathrm{H}^{4}$ & $(\mathrm{POG})_{10}$ & 1.25 \\
\hline $1 \mathrm{~V} 4 \mathrm{~F}^{4}$ & $(\mathrm{POG})_{11}$ & 1.26 \\
\hline $1 \mathrm{YM}^{5}$ & $(\mathrm{OOG})_{9}$ & 1.55 \\
\hline $4 A X Y^{6}$ & $(\mathrm{PPG})_{2}$-PTG-PRG-(PPG) ${ }_{2}$ & 1.24 \\
\hline $2 \mathrm{D}^{2} \mathrm{~F}^{7}$ & $(\mathrm{PPG})_{4}$-POG-(PPG) $)_{4}$ & 1.26 \\
\hline $1 \mathrm{X} 1 \mathrm{~K}^{8}$ & $(\mathrm{PPG})_{4}-\mathrm{PaOG}-(\mathrm{PPG})_{4}$ & 1.1 \\
\hline $3 \mathrm{ABN}^{9}$ & $(\mathrm{PPG})_{4}-\mathrm{ODG}-(\mathrm{PPG})_{4}$ & 1.02 \\
\hline $3 \mathrm{~A} 08^{10}$ & $(\mathrm{PPG})_{4}-\mathrm{OOG}-(\mathrm{PPG})_{4}$ & 1.22 \\
\hline $3{\mathrm{~A} O \mathrm{~A}^{1}}^{1}$ & $(\mathrm{PPG})_{4}$-OPG-(PPG) $)_{4}$ & 1.36 \\
\hline 3 ADM $^{11}$ & $(\mathrm{PPG})_{4}-\mathrm{OSG}-(\mathrm{PPG})_{4}$ & 1.18 \\
\hline $3 \mathrm{~A} 1 \mathrm{H}^{11}$ & $(\mathrm{PPG})_{4}-\mathrm{OTG}-(\mathrm{PPG})_{4}$ & 1.08 \\
\hline $3 \mathrm{~A} 0 \mathrm{M}^{11}$ & $(\mathrm{PPG})_{4}-\mathrm{OVG}-(\mathrm{PPG})_{4}$ & 1.02 \\
\hline $3 \mathrm{WN} 8^{12}$ & $(\mathrm{POG})_{3}-\mathrm{PRG}-(\mathrm{POG})_{4}$ & 1.45 \\
\hline $5 \mathrm{~K} 86^{13}$ & $(\mathrm{POG})_{3}-\mathrm{PRazG}-(\mathrm{POG})_{4}$ & 1.13 \\
\hline $6 \mathrm{M} 80^{14}$ & $\begin{array}{c}(\mathrm{POG})_{2} \text {-azPOG-PRazG- } \\
(\mathrm{POG})_{4}\end{array}$ & 1.1 \\
\hline $1 \mathrm{Q}^{2} \mathrm{D}^{15}$ & $(\mathrm{POG})_{2}$-GFO-GER-(POG) ${ }_{3}$ & 1.8 \\
\hline $4 Z 1 R^{16}$ & $(\mathrm{POG})_{4}-(\mathrm{POA})-(\mathrm{POG})_{5}$ & 1.27 \\
\hline 1QSU 17 & $(\mathrm{POG})_{4}-\mathrm{EKG}-(\mathrm{POG})_{5}$ & 1.75 \\
\hline $2 \mathrm{DRT}^{18}$ & $(\mathrm{POG})_{4}$-LOG-(POG) $)_{5}$ & 1.6 \\
\hline $2 \mathrm{DRX}^{18}$ & $(\mathrm{POG})_{4}-(\mathrm{LOG})_{2}-(\mathrm{POG})_{4}$ & 1.4 \\
\hline $3 \mathrm{~B}_{2} \mathrm{C}^{19}$ & $(\mathrm{POG})_{4-}(\mathrm{aOPG})_{2}-(\mathrm{POG})_{4}$ & 1.36 \\
\hline $1 \mathrm{BKV}^{20}$ & $\begin{array}{c}\text { (POG) })_{3} \text {-ITGARGLAG- } \\
(\mathrm{POG})_{4}\end{array}$ & 2.0 \\
\hline
\end{tabular}

Table S1. Collagen crystal structures and their peptide sequences from the Protein Data Bank used for analyses. 

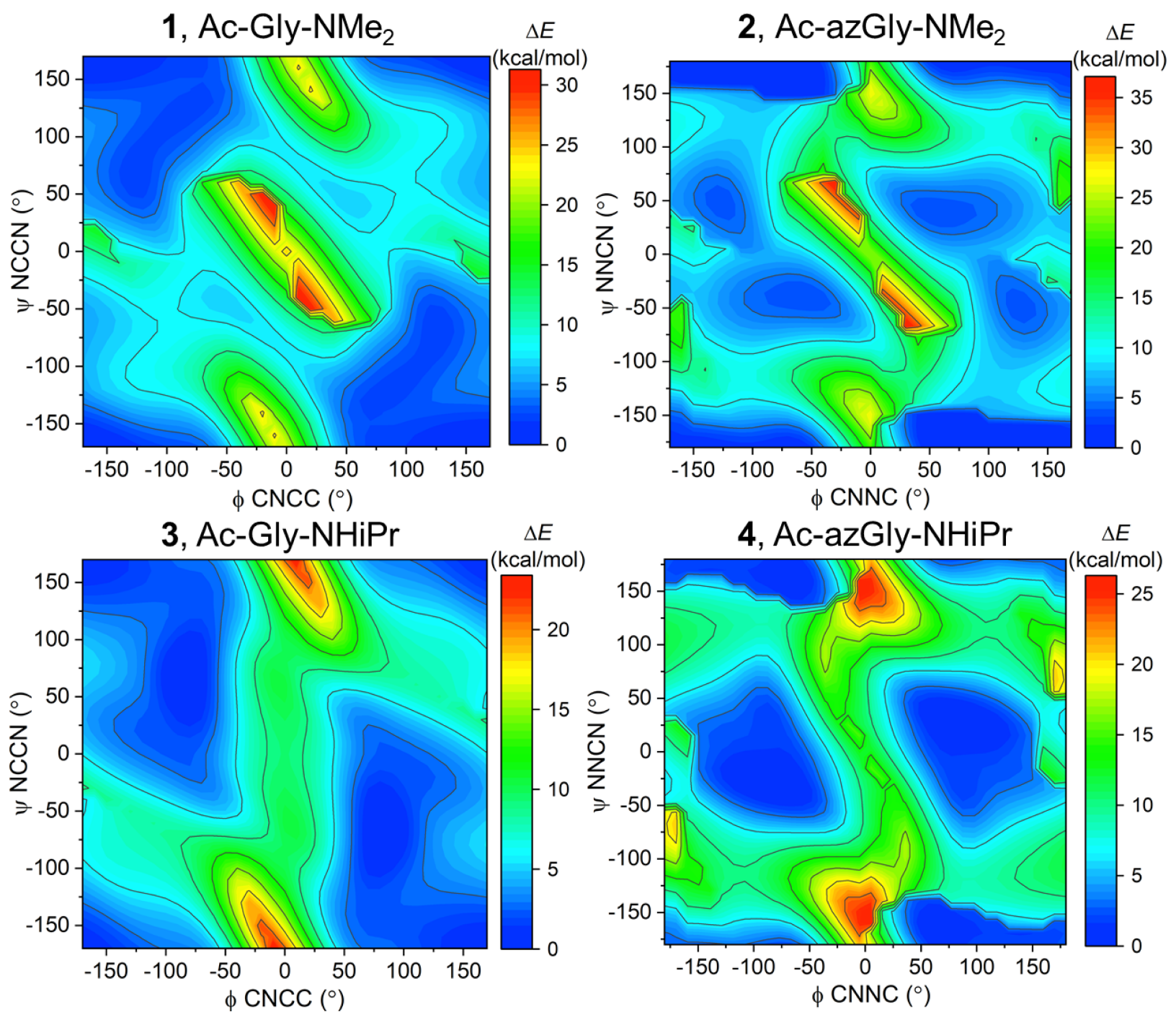

Figure S1. Theoretical Ramachandran plots of the main-chain dihedrals in 1-4. Gas phase energies. 


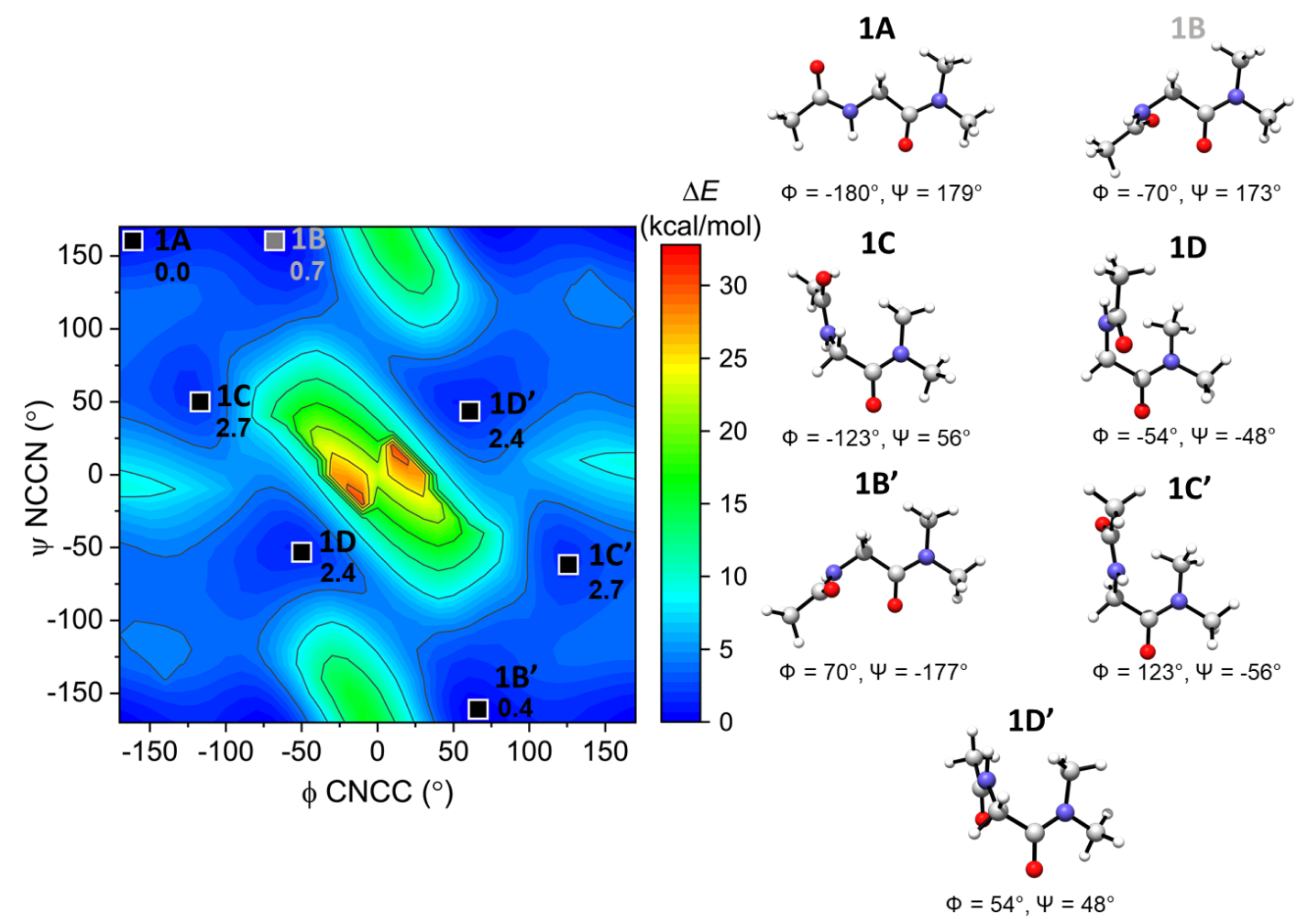

Figure S2. Theoretical Ramachandran plot of Ac-Gly-NMe 2 (1) overlaid the minima (squares) and their relative energies in $\mathrm{kcal} / \mathrm{mol}$. Gray square is the collagen relevant conformation. Structural backbones of minima with main-chain dihedrals shown to the right.

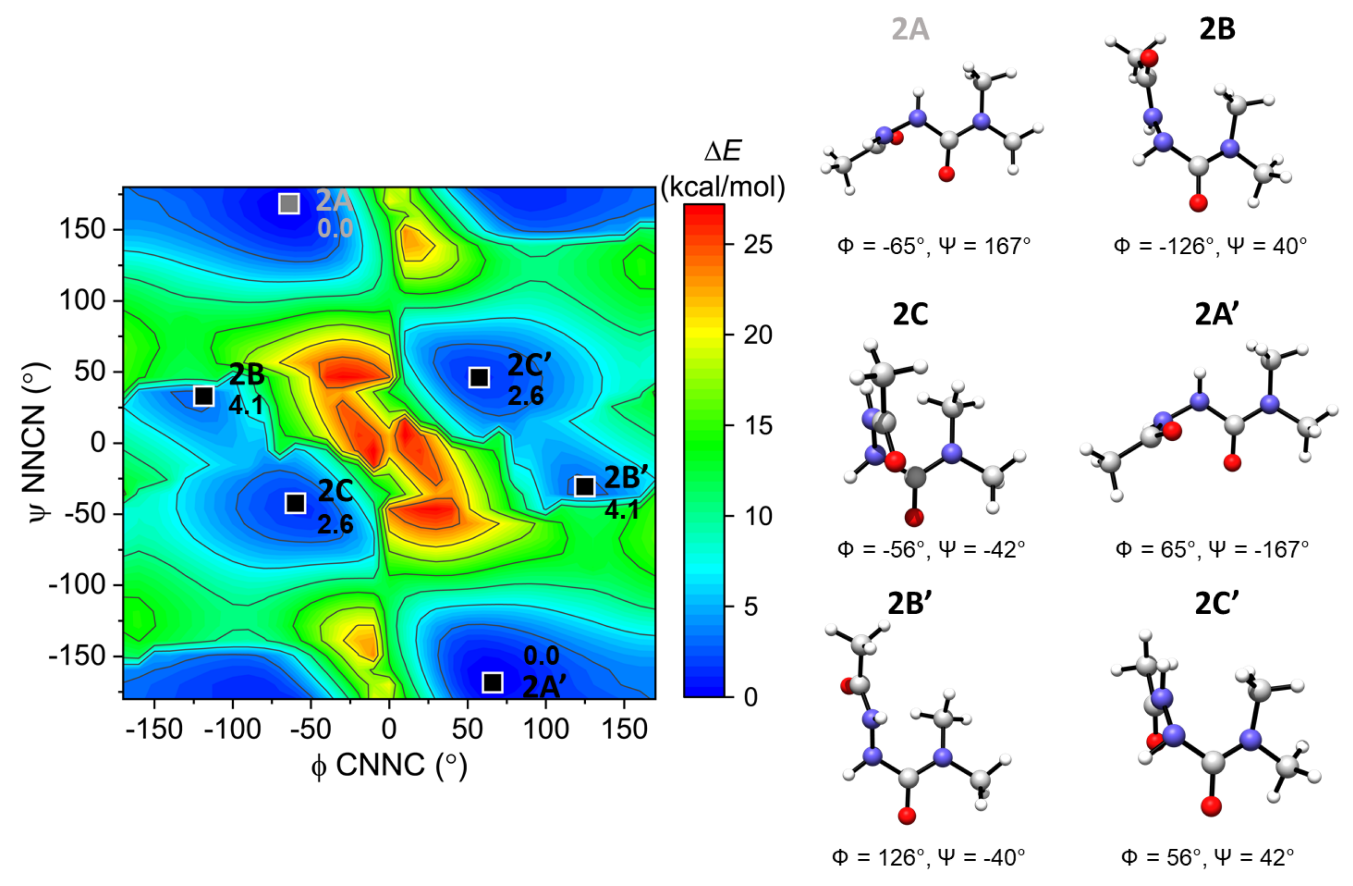

Figure S3. Theoretical Ramachandran plot of Ac-azGly-NMe 2 (2) overlaid the minima (squares) and their relative energies in $\mathrm{kcal} / \mathrm{mol}$. Gray square is the collagen relevant conformation. Structural backbones of minima with main-chain dihedrals shown to the right. 
3A

$\Delta E$ $(\mathrm{kcal} / \mathrm{mol})$

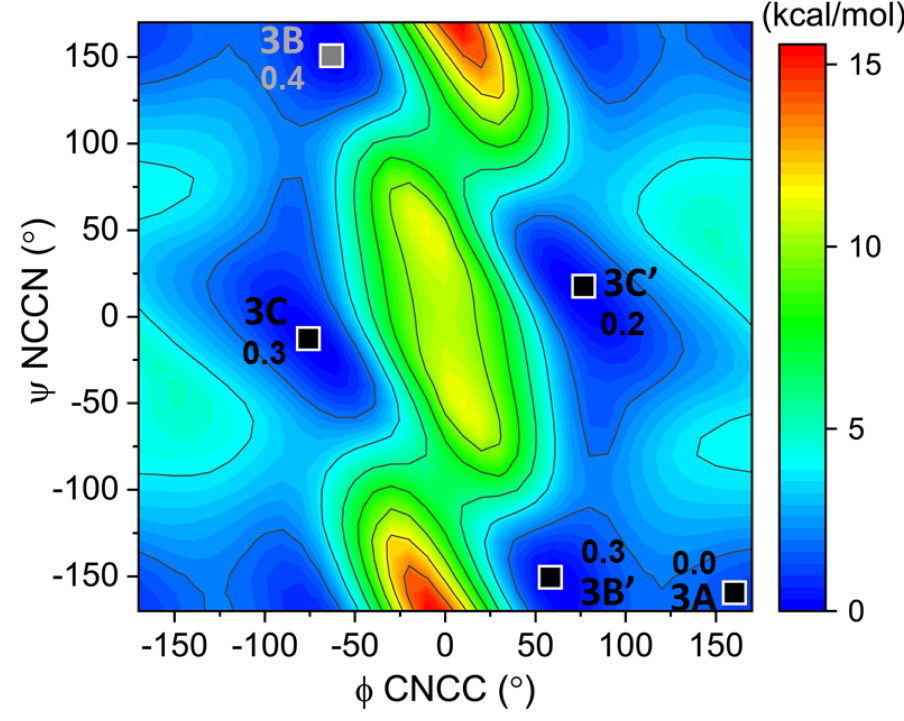

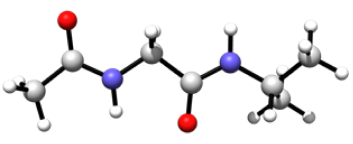

$\Phi=178^{\circ}, \Psi=-179^{\circ}$

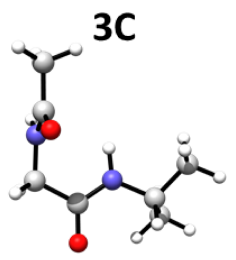

$\Phi=-77^{\circ}, \Psi=-10^{\circ}$

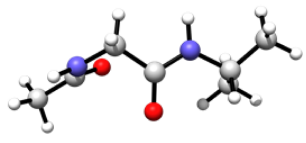

$\Phi=-60^{\circ}, \Psi=150^{\circ}$

3B'

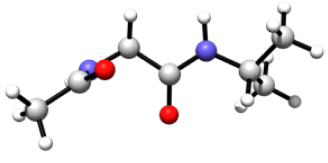

$\Phi=62^{\circ}, \Psi=-153^{\circ}$

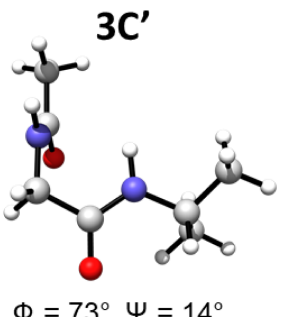

$\Phi=73^{\circ}, \Psi=14^{\circ}$

Figure S4. Theoretical Ramachandran plot of Ac-Gly-NHiPr (3) overlaid the minima (squares) and their relative energies in $\mathrm{kcal} / \mathrm{mol}$. Gray square is the collagen relevant conformation. Structural backbones of minima with main-chain dihedrals shown to the right.

$\Delta E$
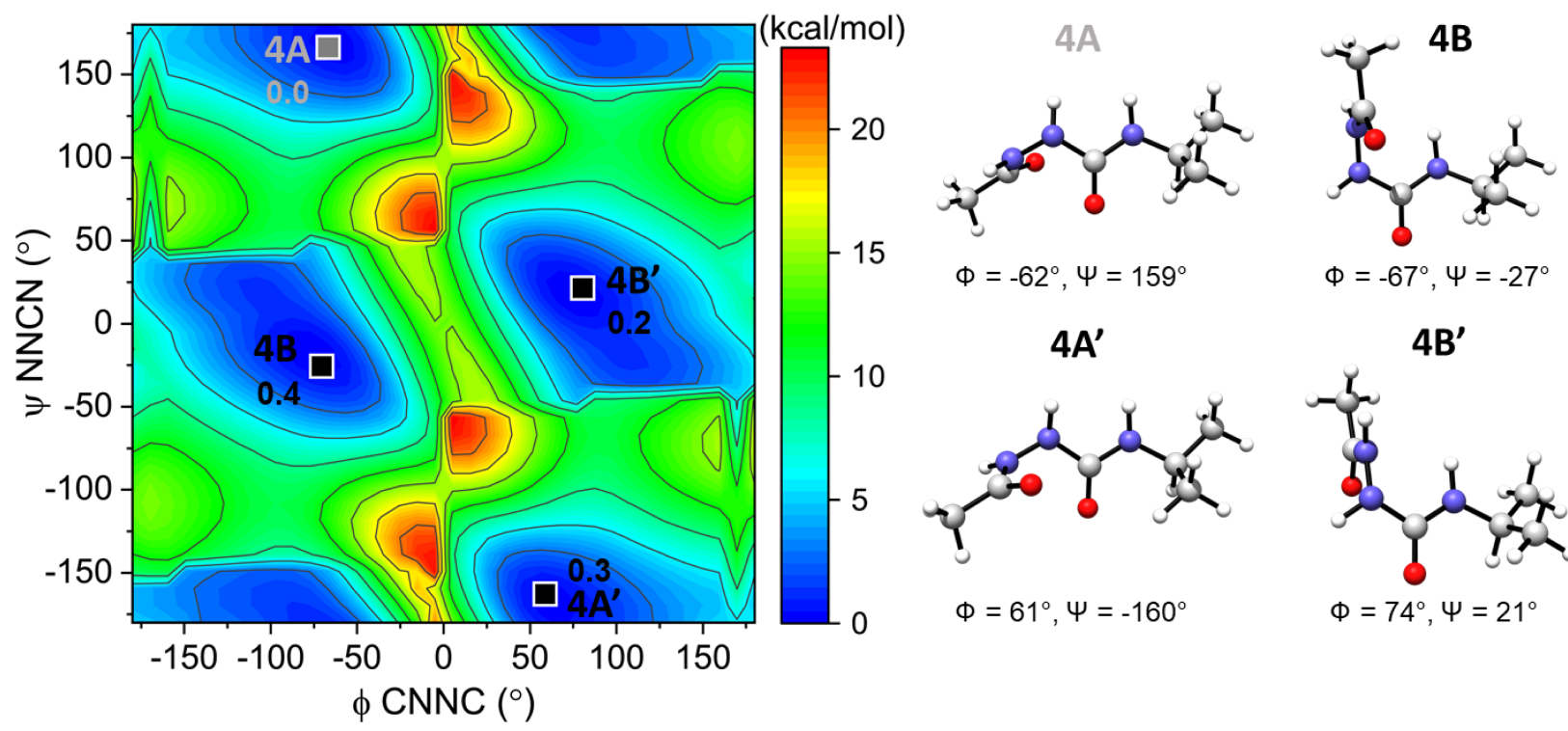

$\Phi=-62^{\circ}, \Psi=159^{\circ}$

$4 A^{\prime}$

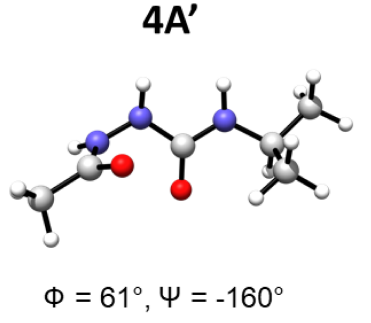

$$
\Phi=-67^{\circ}, \Psi=-27^{\circ}
$$

4B'

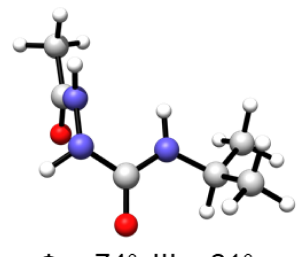

Figure S5. Theoretical Ramachandran plot of Ac-azGly-NHiPr (4) overlaid the minima (squares) and their relative energies in $\mathrm{kcal} / \mathrm{mol}$. Gray square is the collagen relevant conformation. Structural backbones of minima with main-chain dihedrals shown to the right. 


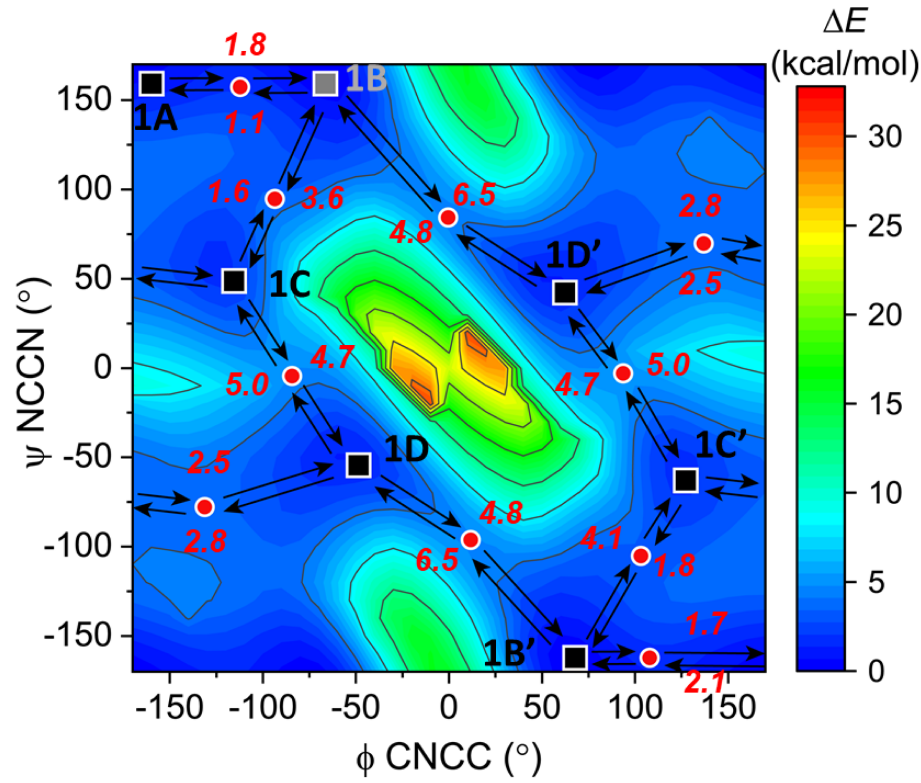

\begin{tabular}{|c|c|c|c|}
\hline Reactant & Product & $\begin{array}{c}\text { Barrier } \\
\text { (kcal/mol) }\end{array}$ & TS \\
\hline $1 \mathrm{~A}$ & $1 \mathrm{~B}$ & 1.8 & $1 \mathrm{AB}$ \\
\hline $1 \mathrm{~A}$ & 1B' & 2.1 & $1 \mathrm{AB}^{\prime}$ \\
\hline $1 \mathrm{~B}$ & $1 \mathrm{C}$ & 3.6 & $1 \mathrm{BC}$ \\
\hline $1 \mathrm{~B}$ & 1D' & 6.5 & $1 \mathrm{BD}^{\prime}$ \\
\hline $1 \mathrm{C}$ & 1D & 4.7 & $1 C D$ \\
\hline $1 \mathrm{C}$ & 1D' & 2.5 & $1 \mathrm{CD}^{\prime}$ \\
\hline $1 \mathrm{D}$ & $1 \mathrm{~B}^{\prime}$ & 4.8 & $1 \mathrm{DB}^{\prime}$ \\
\hline $1 \mathrm{D}$ & $1 C^{\prime}$ & 2.8 & 1DC' \\
\hline 1D' & $1 C^{\prime}$ & 5.0 & 1D'C \\
\hline 1B' & $1 C^{\prime}$ & 4.1 & $1 \mathrm{~B}^{\prime} \mathrm{C}^{\prime}$ \\
\hline
\end{tabular}

Figure S6. Theoretical Ramachandran plot of $\mathrm{Ac}-\mathrm{Gly}-\mathrm{NMe}_{2}$ (1) overlaid the minima (squares) and transition states (red circles) for conformational rotations. Gray square is the collagen relevant conformation. Barriers (red numbers) for conformational rotations are in kcal/mol.

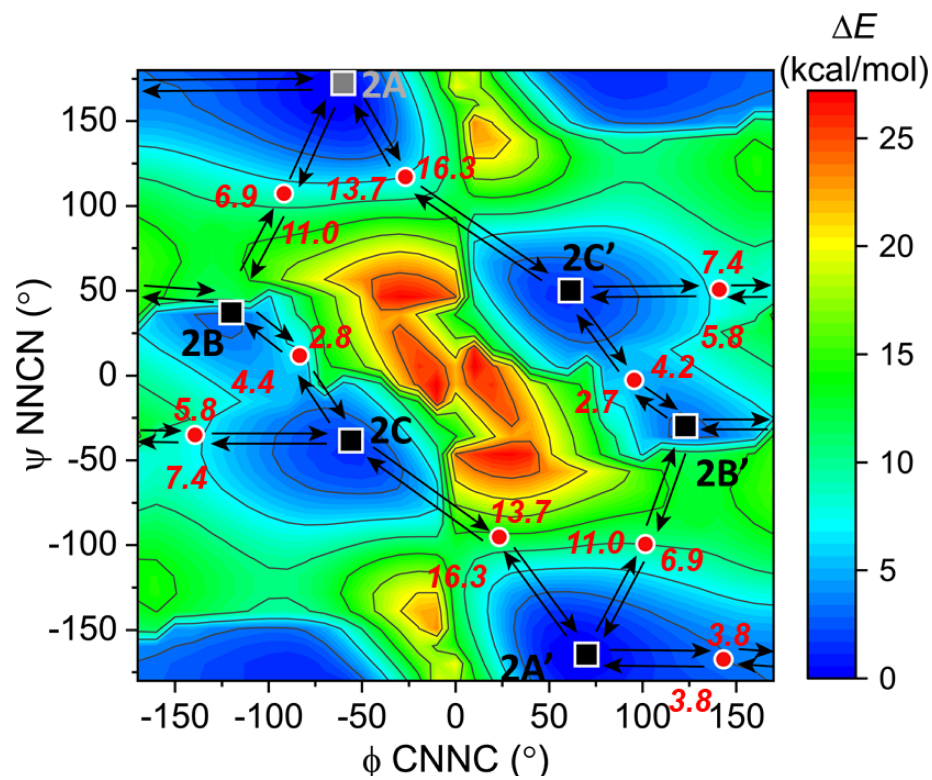

\begin{tabular}{|c|c|c|c|}
\hline Reactant & Product & $\begin{array}{c}\text { Barrier } \\
\text { (kcal/mol) }\end{array}$ & TS \\
\hline $2 \mathrm{~A}$ & $2 \mathrm{~B}$ & 11.0 & $2 \mathrm{AB}$ \\
\hline $2 \mathrm{~A}$ & $2 \mathrm{~A}^{\prime}$ & 3.8 & $2 \mathrm{~A}^{\prime}$ \\
\hline $2 \mathrm{~A}$ & $2 \mathrm{C}^{\prime}$ & 16.3 & $2 \mathrm{AC}^{\prime}$ \\
\hline $2 \mathrm{~B}$ & $2 \mathrm{C}$ & 2.8 & $2 \mathrm{BC}$ \\
\hline $2 \mathrm{~B}$ & $2 \mathrm{C}^{\prime}$ & 5.8 & $2 \mathrm{BC}^{\prime}$ \\
\hline $2 \mathrm{C}$ & $2 \mathrm{~A}^{\prime}$ & 13.7 & $2 \mathrm{CA}^{\prime}$ \\
\hline $2 \mathrm{C}$ & $2 \mathrm{~B}^{\prime}$ & 7.4 & $2 \mathrm{CB}^{\prime}$ \\
\hline $2 \mathrm{~A}^{\prime}$ & $2 \mathrm{~B}^{\prime}$ & 11.0 & $2 \mathrm{~A}^{\prime} \mathrm{B}^{\prime}$ \\
\hline $2 \mathrm{~B}^{\prime}$ & $2 \mathrm{C}^{\prime}$ & 2.7 & $2 \mathrm{~B}^{\prime} \mathrm{C}^{\prime}$ \\
\hline
\end{tabular}

Figure S7. Theoretical Ramachandran plot of Ac-azGly-NMe 2 (2) overlaid the minima (squares) and transition states (red circles) for conformational rotations. Gray square is the collagen relevant conformation. Barriers (red numbers) for conformational rotations are in $\mathrm{kcal} / \mathrm{mol}$. 


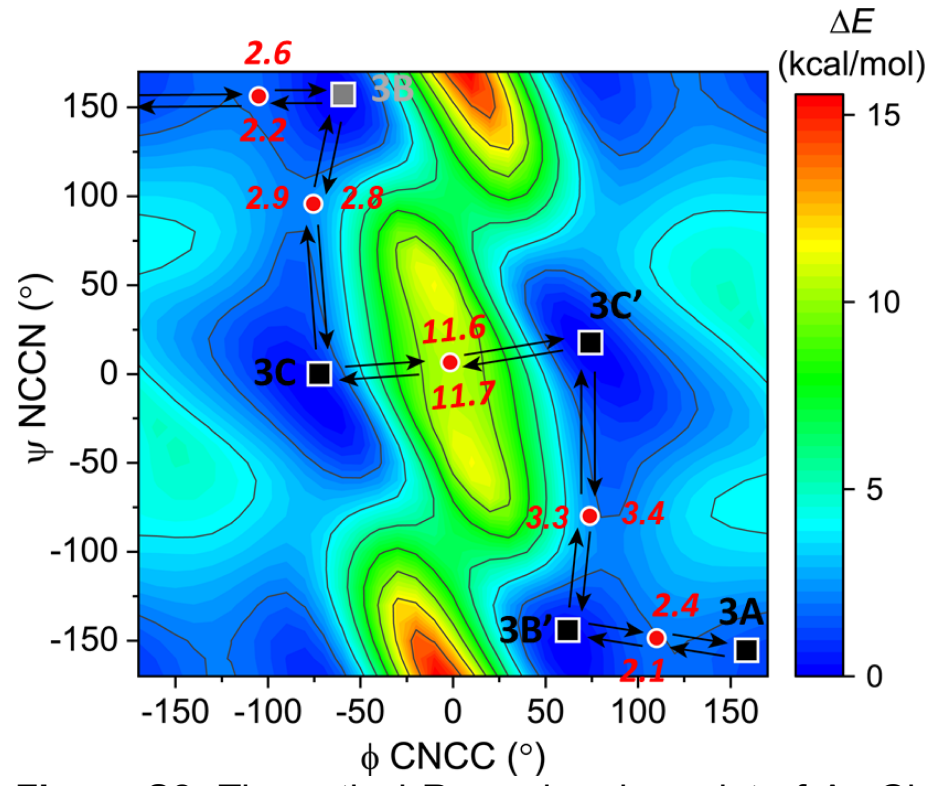

\begin{tabular}{|c|c|c|c|}
\hline Reactant & Product & $\begin{array}{c}\text { Barrier } \\
(\mathbf{k c a l} / \mathbf{m o l})\end{array}$ & TS \\
\hline $3 \mathrm{~A}$ & $3 \mathrm{~B}$ & 2.6 & $3 \mathrm{AB}$ \\
\hline $3 \mathrm{~A}$ & $3 \mathrm{~B}^{\prime}$ & 2.1 & $3 \mathrm{AB}^{\prime}$ \\
\hline $3 \mathrm{~B}$ & $3 \mathrm{C}$ & 2.8 & $3 \mathrm{BC}$ \\
\hline $3 \mathrm{C}$ & $3 \mathrm{C}^{\prime}$ & 11.6 & $3 \mathrm{CC}^{\prime}$ \\
\hline $3 \mathrm{~B}^{\prime}$ & $3 \mathrm{C}^{\prime}$ & 3.3 & $3 \mathrm{~B}^{\prime} \mathrm{C}^{\prime}$ \\
\hline
\end{tabular}

Figure S8. Theoretical Ramachandran plot of Ac-Gly-NHiPr (3) overlaid the minima (squares) and transition states (red circles) for conformational rotations. Gray square is the collagen relevant conformation. Barriers (red numbers) for conformational rotations are in kcal/mol.

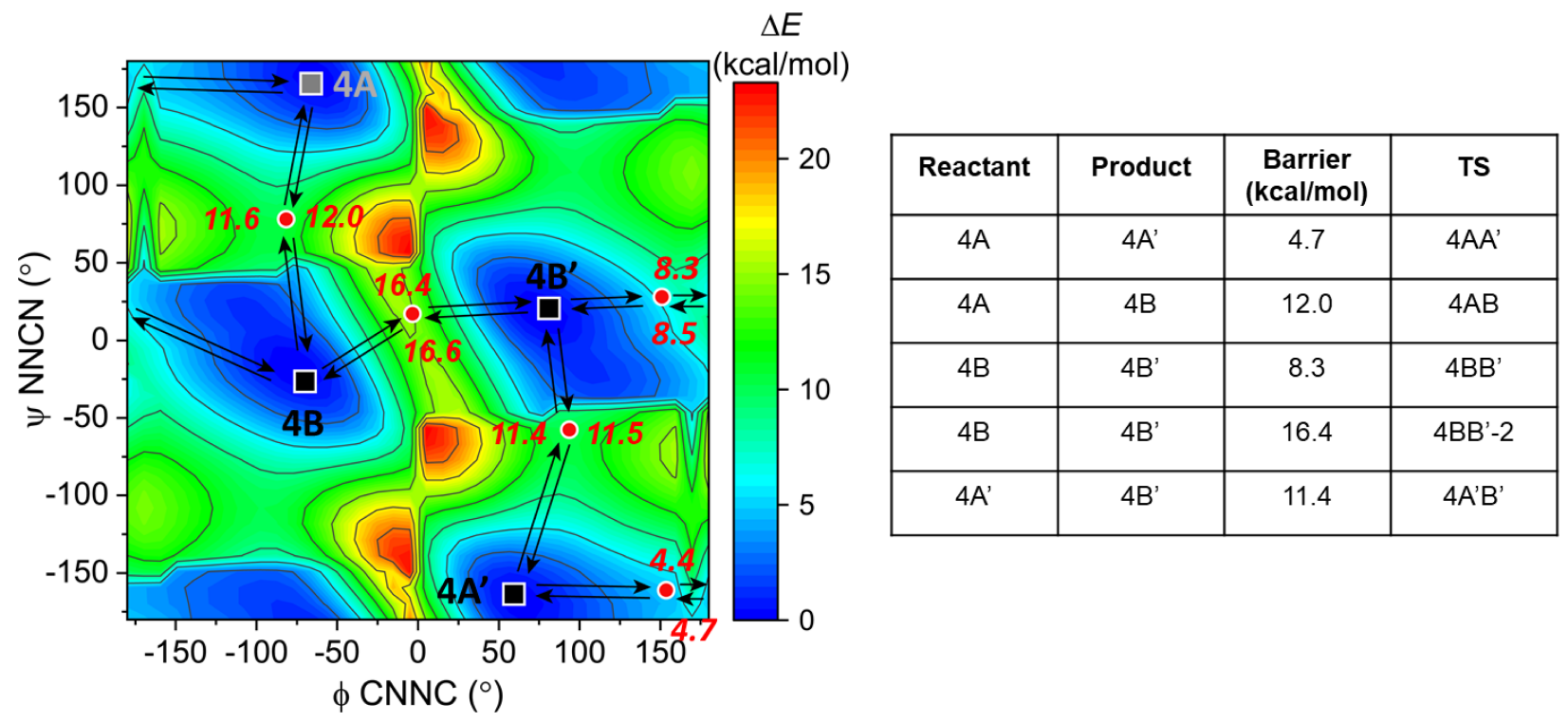

Figure S9. Theoretical Ramachandran plot of Ac-azGly-NHiPr (4) overlaid the minima (squares) and transition states (red circles) for conformational rotations. Gray square is the collagen relevant conformation. Barriers (red numbers) for conformational rotations are in $\mathrm{kcal} / \mathrm{mol}$. 


\begin{tabular}{|c|c|c|}
\hline Amide isomerization & $\boldsymbol{\Delta G ^ { \ddagger }}(\mathbf{k c a l} / \mathbf{m o l})$ & $\boldsymbol{\Delta G}(\mathbf{k c a l} / \mathbf{m o l})$ \\
\hline $1 \mathrm{~A} \rightarrow 1 \mathrm{~A}-E$ & 20.8 & 2.5 \\
\hline 1B $\rightarrow$ 1B-E & $\mathbf{2 0 . 3}$ & $\mathbf{2 . 2}$ \\
\hline $1 \mathrm{C} \rightarrow 1 \mathrm{C}-E$ & 20.4 & 3.5 \\
\hline $1 \mathrm{D} \rightarrow 1 \mathrm{D}^{\prime}-E$ & 20.8 & 2.4 \\
\hline $1 \mathrm{~B}^{\prime} \rightarrow 1 \mathrm{~B}^{\prime}-E$ & 20.4 & 2.5 \\
\hline 1C' $\rightarrow 1 \mathrm{C}^{\prime}-E$ & 20.4 & 3.5 \\
\hline 1D' $\rightarrow 1 \mathrm{D}^{\prime}-E$ & 20.8 & 2.4 \\
\hline
\end{tabular}

Table S2. Amide isomerization barriers and free energies for all minima found for Ac-Gly-NMe 2 (1). Bolded row has collagen relevant dihedrals: 1B $\left(\varphi=-70^{\circ}, \psi=173^{\circ}\right)$; 1B-E $\left(\varphi=-63^{\circ}, \psi=\right.$ $\left.179^{\circ}\right)$. The lowest barriers for isomerization are shown.

\begin{tabular}{|c|c|c|}
\hline Amide isomerization & $\Delta G^{ \pm}(\mathrm{kcal} / \mathrm{mol})$ & $\Delta G(\mathrm{kcal} / \mathrm{mol})$ \\
\hline $2 \mathrm{~A} \rightarrow 2 \mathrm{~A}-\mathrm{E}$ & 19.4 & 1.6 \\
\hline $2 \mathrm{~B} \rightarrow 2 \mathrm{~B}-\mathrm{E}$ & 19.3 & 1.5 \\
\hline $2 \mathrm{C} \rightarrow 2 \mathrm{C}-\mathrm{E}$ & 22.9 & 2.1 \\
\hline $2 A^{\prime} \rightarrow 2 A^{\prime}-E$ & 19.2 & 1.6 \\
\hline $2 \mathrm{~B}^{\prime} \rightarrow 2 \mathrm{~B}^{\prime}-\mathrm{E}$ & 19.3 & 1.5 \\
\hline $2 C^{\prime} \rightarrow 2 C^{\prime}-E$ & 22.9 & 2.1 \\
\hline
\end{tabular}

Table S3. Amide isomerization barriers and free energies for all minima found for Ac-azGly-NMe $\mathrm{N}_{2}$ (2). Bolded row has collagen relevant dihedrals: $2 \mathrm{~A}\left(\varphi=-65^{\circ}, \psi=167^{\circ}\right) ; 2 \mathrm{~A}-\mathrm{E}\left(\varphi=-70^{\circ}, \psi=\right.$ $\left.172^{\circ}\right)$. The lowest barriers for isomerization are shown.

\begin{tabular}{|c|c|c|}
\hline Model systems & $\boldsymbol{\delta}\left(^{\circ}\right)$ & $\boldsymbol{d}(\boldsymbol{A})$ \\
\hline $\mathbf{2}\left(\varphi=-70^{\circ}, \psi=-78^{\circ}\right)$ & -25.4 & 0.374 \\
\hline $\mathbf{2}\left(\varphi=-70^{\circ}, \psi=71^{\circ}\right)$ & -19.7 & 0.326 \\
\hline $\mathbf{4}\left(\varphi=-74^{\circ}, \psi=-72^{\circ}\right)$ & -29.9 & 0.407 \\
\hline $\mathbf{4}\left(\varphi=-74^{\circ}, \psi=107^{\circ}\right)$ & -34.3 & 0.437 \\
\hline
\end{tabular}

Table S4. Pyramidalization parameters of non-native nitrogen in azGly model systems 2 and $\mathbf{4}$. $\delta$ is a measurement of pyramidal character (in degrees) and is defined as $\delta=S-360^{\circ}$, where $S$ is the sum of the valence angles around the atom of interest ( $\alpha \mathrm{N})$. If $\delta=0$, then the site is fully planar. As $\delta$ becomes more negative, the magnitude of pyramidalization increases $\left(\mathrm{sp}^{3}\right.$ character). Another measurement of pyramidalization $(d)$ is defined using a triangular pyramid with the $\alpha \mathrm{N}$ at the apex and substituent atoms positioned at the remaining vertices of the base. The distance from the apex normal to the base plane is measured as the $d$ value, in $\AA$. 

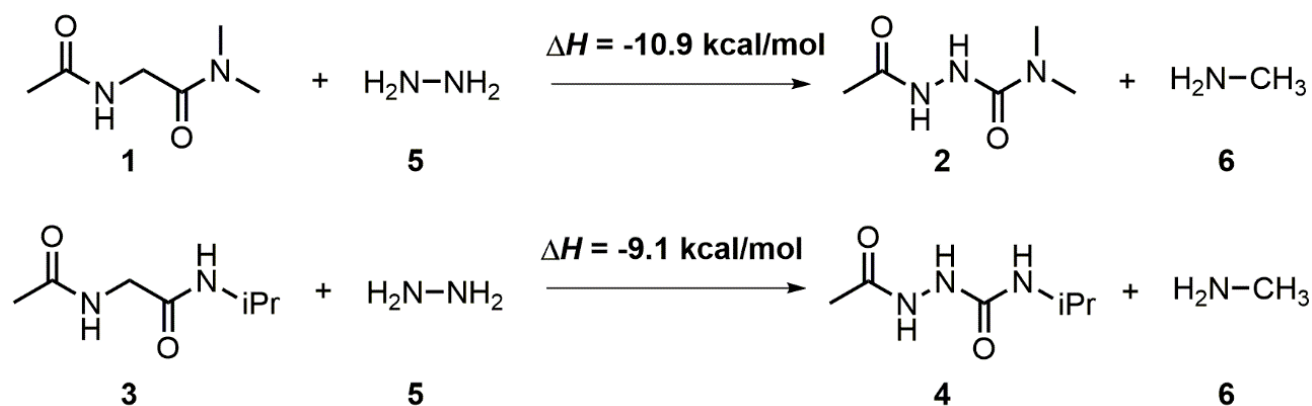

Scheme S1. Isodesmic equations for determining the stabilities of 1-4. Dihedrals used: $\varphi=-70^{\circ}$ (1-6), $\psi=170^{\circ}(1-4)$. Gas phase energies.
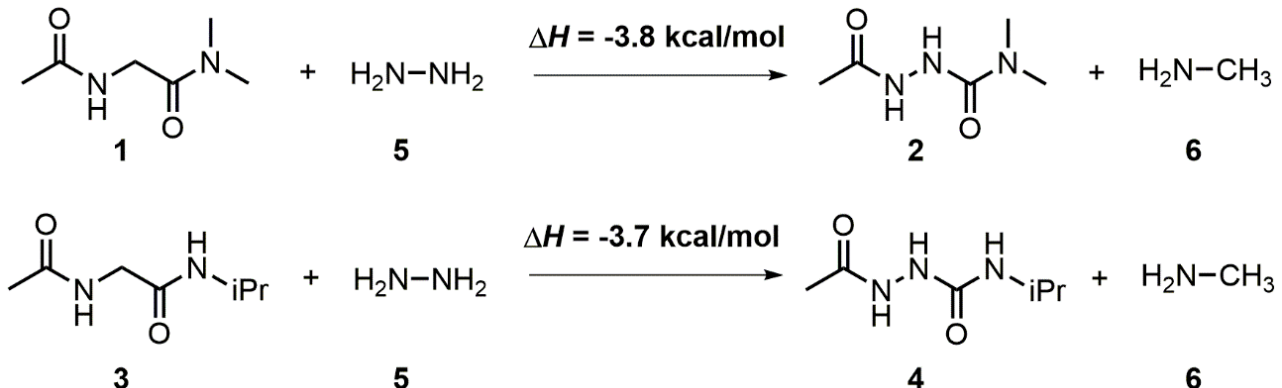

Scheme S2. Isodesmic equations for determining the stabilities of 1-4. All minima. Dihedrals used: $\varphi=-70^{\circ}, \psi=173^{\circ}(1) ; \varphi=-65^{\circ}, \psi=-178^{\circ}(2) ; \varphi=-60^{\circ}, \psi=150^{\circ}(3) ; \varphi=-62^{\circ}, \psi=159^{\circ}$ $(4) ; \varphi=-31^{\circ}(5) ; \varphi=-57^{\circ}(6)$.

\begin{tabular}{|c|c|c|c|}
\hline Compound & $\begin{array}{c}E(2) \\
\text { (kcal/mol) }\end{array}$ & $\begin{array}{c}\mathrm{n}_{\mathrm{O}} \rightarrow \mathrm{m}^{*} \mathrm{C}=\mathrm{O} \\
\text { Angle } \angle \mathrm{OCO}\left({ }^{\circ}\right)\end{array}$ & $\begin{array}{c}\mathrm{n}_{0} \rightarrow \pi^{*} \mathrm{c}=0 \\
\text { Distance }(\AA)\end{array}$ \\
\hline 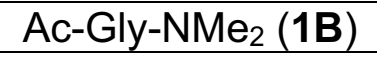 & Not detected & 81 & 3.1 \\
\hline $\begin{array}{c}\text { Ac-azGly-NMe } \\
(2 A)\end{array}$ & Not detected & 85 & 3.0 \\
\hline Ac-Gly-NHiPr (3B) & 0.8 & 93 & 2.9 \\
\hline $\begin{array}{c}\text { Ac-azGly-NHiPr } \\
\text { (4A) }\end{array}$ & 0.5 & 88 & 2.9 \\
\hline
\end{tabular}

Table S5. Second order perturbation energies of $n_{0} \rightarrow \pi^{*}{ }_{C}=0$ interactions of $1-4$ at optimized minima with collagen relevant dihedrals. 


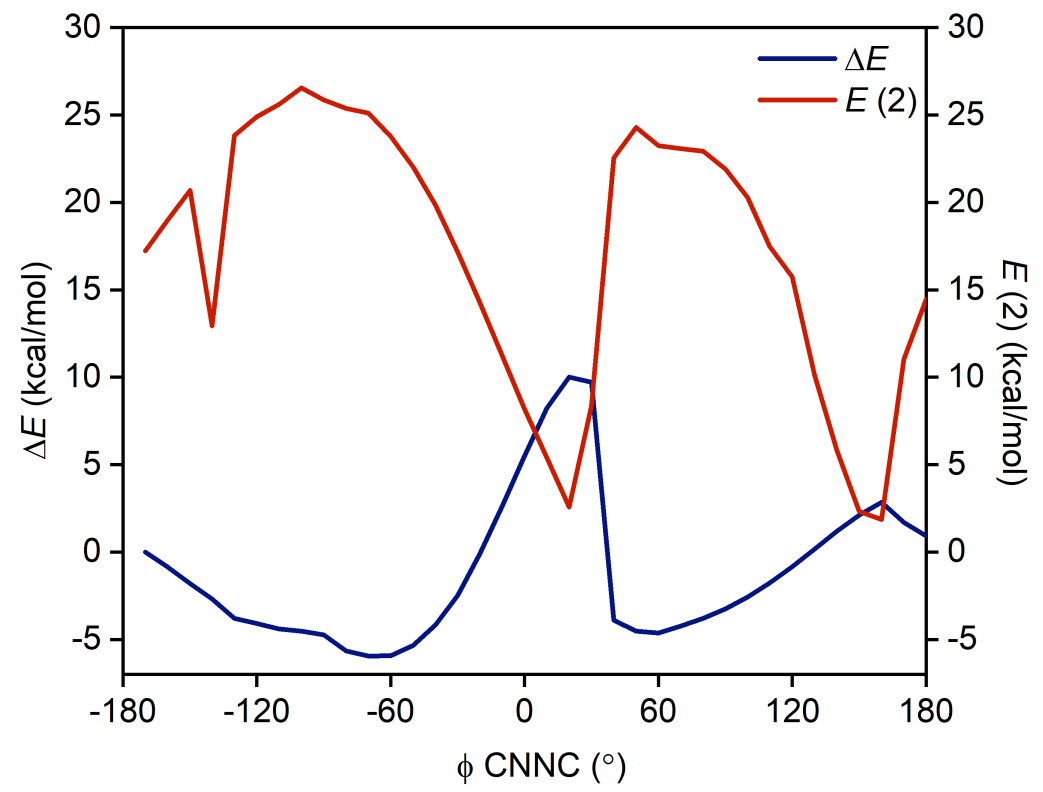

Figure $\mathbf{S 1 0 .} 360^{\circ}$ relaxed $\varphi$ dihedral scan of 2 vs total second order interactions $\left(n_{N} \rightarrow \sigma^{*}{ }_{N-C}\right.$ and $\mathrm{n}_{\mathrm{N}} \rightarrow \sigma^{*}{ }_{\mathrm{N}-\mathrm{C}}$ ) from NBO analysis.

A

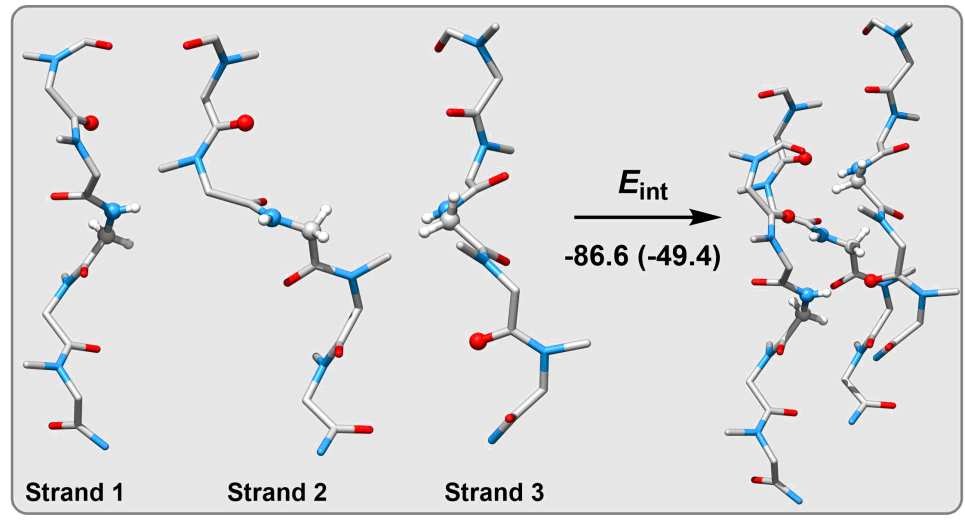

B

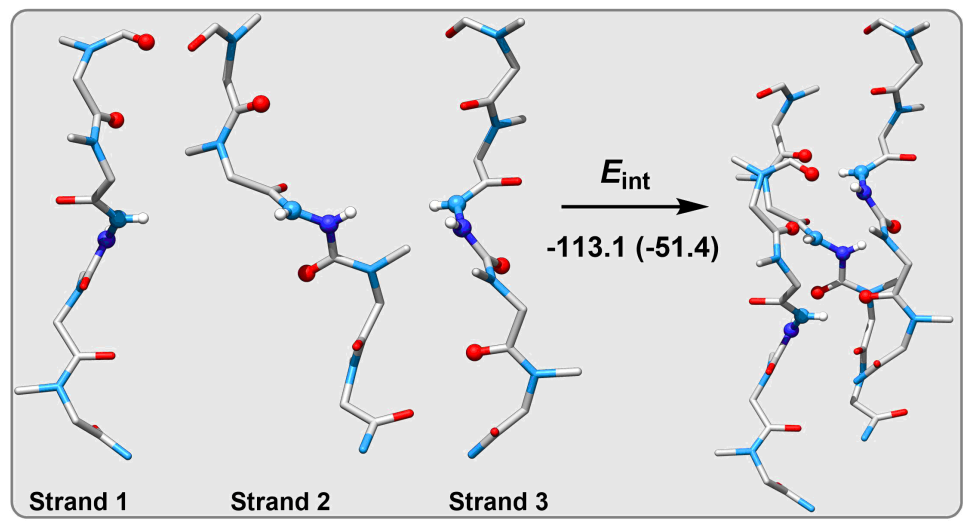

Figure S11. Interaction analysis of truncated triple helices from PDB 5K86 at wB97X-D/6$311+G(d, p)$ level of theory. IEFPCM solvation corrections (water) are in parentheses. Energies are in $\mathrm{kcal} / \mathrm{mol}$. 


\begin{tabular}{|c|c|}
\hline Gly Triple Helix (Gly N-H) & Natural charge (e) \\
\hline H28 & $0.39(0.39)$ \\
\hline $\mathrm{H} 81$ & $0.40(0.40)$ \\
\hline $\mathrm{H} 134$ & $0.39(0.39)$ \\
\hline & \\
\hline azGly Triple Helix (azGly N-H) & Natural charge (e) \\
\hline $\mathrm{H} 20$ & $0.41(0.41)$ \\
\hline $\mathrm{H} 21$ & $0.41(0.42)$ \\
\hline $\mathrm{H} 59$ & $0.40(0.41)$ \\
\hline $\mathrm{H} 60$ & $0.41(0.41)$ \\
\hline $\mathrm{H} 98$ & $0.40(0.41)$ \\
\hline $\mathrm{H} 99$ & $0.41(0.42)$ \\
\hline
\end{tabular}

Table S6. NBO charges on $\mathrm{H}$ atoms in Gly and azGly in the triple helix. Calculated at M06$2 X(D 3) / 6-311+G(d, p)$. SMD solvation corrections (water) are in parentheses.

\begin{tabular}{|c|c|c|c|}
\hline & $\begin{array}{c}E(2) \\
\text { (kcal/mol) }\end{array}$ & $\begin{array}{c}\text { no } \rightarrow \pi^{*} \mathrm{C}=\mathrm{O} \\
\text { Angle } \angle \text { OCO }\left(^{\circ}\right)\end{array}$ & $\begin{array}{c}\text { no } \rightarrow \pi^{*} c=0 \\
\text { Distance }(\AA)\end{array}$ \\
\hline \multicolumn{4}{|l|}{ Strand 1} \\
\hline $\mathrm{n}_{\mathrm{O} 7} \rightarrow \pi^{*}{ }_{\mathrm{C} 16-\mathrm{O} 17}$ & 1.3 & 93 & 2.9 \\
\hline $\mathrm{n}_{\mathrm{O} 27} \rightarrow \pi^{*}{ }_{\mathrm{C} 33-\mathrm{O} 34}$ & 1.5 & 94 & 2.8 \\
\hline $\mathrm{n}_{34} \rightarrow \pi^{*}{ }^{\mathrm{C}} 43-\mathrm{O} 44$ & 1.0 & 96 & 2.9 \\
\hline \multicolumn{4}{|l|}{ Strand 2} \\
\hline $\mathrm{n}_{055} \rightarrow \Pi^{*}{ }{ }_{59}-060$ & 0.5 & 88 & 3.0 \\
\hline no60 $\rightarrow \pi^{*}{ } 669-070$ & 1.4 & 91 & 2.8 \\
\hline \multicolumn{4}{|l|}{ Strand 3} \\
\hline $\mathrm{n} 0113 \rightarrow \Pi^{*}{ }^{2} 122-0123$ & 1.3 & 88 & 2.8 \\
\hline $\mathrm{n}_{1133} \rightarrow \Pi^{*}{ }_{\mathrm{C}} 139-0140$ & 0.9 & 89 & 3.0 \\
\hline $\mathrm{n}_{0140} \rightarrow \Pi^{*} \mathrm{C} 149-0150$ & 1.6 & 94 & 2.8 \\
\hline Total & 8.5 & & \\
\hline
\end{tabular}

Table S7. Second order perturbation energies of $n_{0} \rightarrow \pi^{*} \mathrm{C}=0$ interactions in the triple helix containing Gly. 


\begin{tabular}{|c|c|c|c|}
\hline & $\begin{array}{c}E(2) \\
\text { (kcal/mol) }\end{array}$ & $\begin{array}{c}\text { no } \rightarrow \pi^{*} \mathrm{C}=\mathrm{O} \\
\text { Angle } \angle \text { OCO }\left(^{\circ}\right)\end{array}$ & $\begin{array}{c}\text { no } \rightarrow \pi^{*} c=0 \\
\text { Distance }(\AA)\end{array}$ \\
\hline \multicolumn{4}{|l|}{ Strand 1} \\
\hline $\mathrm{n}_{\mathrm{O} 6} \rightarrow \pi^{*}{ }_{\mathrm{C} 13-\mathrm{O} 14}$ & 0.6 & 104 & 3.0 \\
\hline \multicolumn{4}{|l|}{ Strand 2} \\
\hline $\mathrm{n}_{\mathrm{O} 41} \rightarrow \Pi^{*}{ }^{\mathrm{C}} 44-\mathrm{O} 45$ & 0.9 & 84 & 2.9 \\
\hline no56 $\rightarrow \pi^{*}{ }^{*} 63-064$ & 0.8 & 93 & 2.9 \\
\hline $\mathrm{n}_{\mathrm{O} 64} \rightarrow \Pi^{*}{ }^{\mathrm{C}} 71-\mathrm{O} 72$ & 1.4 & 90 & 2.8 \\
\hline \multicolumn{4}{|l|}{ Strand 3} \\
\hline no80 $\rightarrow \Pi^{*}{ }^{*} 83-084$ & 0.9 & 87 & 2.9 \\
\hline no84 $\rightarrow \pi^{*}{ }^{\circ} 91-092$ & 1.1 & 97 & 2.9 \\
\hline $\mathrm{n}_{0103} \rightarrow \pi^{*} \mathrm{C} 110-0111$ & 0.9 & 93 & 2.9 \\
\hline Total & 6.6 & & \\
\hline
\end{tabular}

Table S8. Second order perturbation energies of $n_{0} \rightarrow \pi^{*}{ }_{C}=0$ interactions in the triple helix containing azGly.

\section{Calculated Geometries}

Coordinates with their respective energies (in hartree) and number of imaginary frequencies.

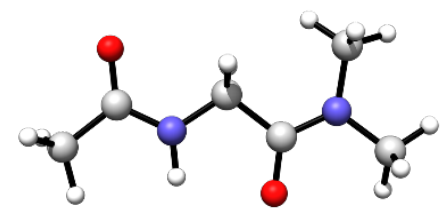

$1 \mathrm{~A}$

01

\# imaginary freq. $=0$

$E=-495.678899$

$7-1.348856-0.351765-0.002366$

$1-1.222245-1.3571540 .029064$

$6-0.1606580 .467681-0.042497$

$1-0.1637781 .102124-0.935296$

$1-0.1221341 .1310700 .829312$

$6-2.5753640 .1880170 .003815$

$6-3.741190-0.7607240 .049503$

$1-4.356690-0.596121-0.838445$

$1-3.432812-1.8063550 .089867$

$1-4.346351-0.5245510 .928187$

$8-2.7434461 .421514-0.028870$

$61.062781-0.433703-0.048350$

$80.930175-1.669612-0.003080$

$72.2661870 .156839-0.110723$

$63.448059-0.6879460 .034951$

$14.322484-0.115790-0.273756$
$13.576813-1.0076481 .074730$

$13.356806-1.571148-0.597179$

62.4544561 .5938310 .071324

12.5613941 .8442041 .132298

$13.3614951 .892966-0.455058$

$11.6213592 .154734-0.348977$

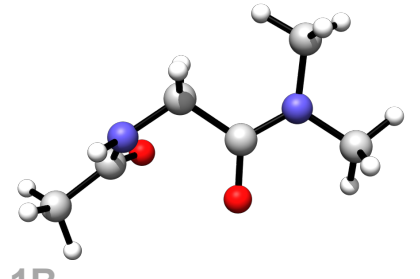

01

\# imaginary freq. $=0$

$E=-495.678941$

$71.4685360 .690721-0.657300$

$11.7783650 .714894-1.619718$

$60.1005071 .030644-0.357060$

10.0633331 .5795760 .586726

$1-0.2757071 .687750-1.144348$

62.2432440 .0735920 .254981

$63.611853-0.359821-0.190537$

$13.685026-1.443732-0.070213$

$13.821390-0.095095-1.227602$

14.3545050 .1033440 .463521

$81.850415-0.1123461 .418920$

$6-0.793477-0.208528-0.266336$

$8-0.352264-1.332365-0.555015$

$7-2.062702-0.0255980 .140810$ 
$6-2.964634-1.1728230 .101198$

$1-3.250468-1.412942-0.928910$

$1-2.485026-2.0441530 .546800$

$1-3.860661-0.9271470 .671462$

$6-2.7019011 .2842900 .231164$

$1-3.4087251 .2737201 .062766$

$1-1.9742042 .0714510 .414923$

$1-3.2474211 .510038-0.691677$

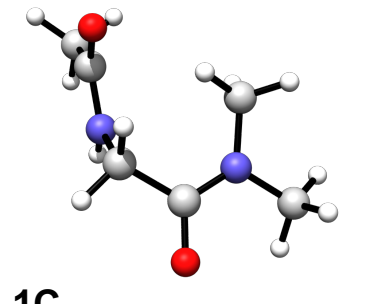

$1 \mathrm{C}$

01

\# imaginary freq. $=0$

$E=-495.675996$

$71.066140-0.7372360 .422858$

$10.967415-0.8711101 .422085$

$60.029754-1.270506-0.439013$

$10.273064-1.015373-1.472708$

$1-0.011772-2.357825-0.355874$

$62.142534-0.060585-0.023332$

63.0820190 .4668971 .025876

13.0250541 .5591791 .013834

12.8425390 .1056932 .026718

14.1014370 .1778710 .762145

$82.3481010 .139016-1.231557$

$6-1.359884-0.757998-0.073004$

$8-2.257478-1.5802070 .193612$

$7-1.5714960 .564925-0.029374$

$6-2.8687771 .0576080 .420909$

$1-3.5863461 .089893-0.405666$

$1-3.2605330 .4122521 .206770$

$1-2.7360352 .0655410 .816267$

$6-0.6273461 .572193-0.504412$

$1-0.1040312 .0487650 .330999$

$10.1004901 .139938-1.189139$

$1-1.1885722 .335240-1.048409$

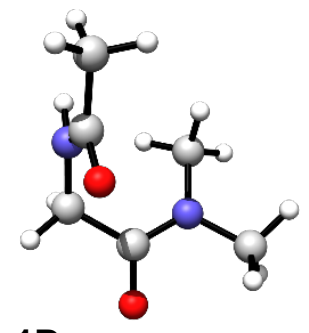

01

$\#$ imaginary freq. $=0$

$E=-495.676727$

$71.270149-0.1151560 .944414$

11.5418020 .6055651 .600620

$60.109257-0.9331671 .234292$

$10.366654-1.9923761 .173323$

$1-0.213961-0.7177862 .255314$

$61.846475-0.131050-0.271255$

$62.9684460 .839764-0.509603$

$12.6781051 .506144-1.326144$

13.2081761 .4319750 .374381

$13.8523050 .281539-0.827226$

$81.461261-0.916637-1.153840$

$6-1.061456-0.7307710 .273658$

$8-1.620145-1.732337-0.212763$

$7-1.4640240 .516797-0.008675$

$6-2.5040100 .713753-1.013157$

$1-2.3669111 .697279-1.465104$

$1-2.422481-0.049908-1.786153$

$1-3.5015830 .663466-0.564181$

$6-0.9965881 .7171010 .680620$

$1-0.6355151 .4824211 .681445$

$1-0.2067082 .2207260 .114026$

$1-1.8444252 .3974840 .785012$

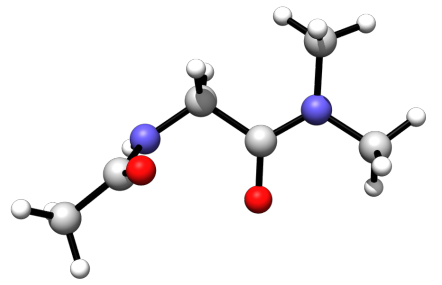

1B'

01

\# imaginary freq. $=0$

$E=-495.678857$

7 -1.477707 $0.645944-0.704782$

$1-1.8268680 .609580-1.653353$

$6-0.0998941 .004382-0.482172$

$10.2396061 .623816-1.315034$

$1-0.0240961 .5999650 .431430$

$6-2.2143740 .0860460 .273719$

$6-3.592668-0.386144-0.095964$

$1-4.3143080 .0707290 .585043$

$1-3.856806-0.146739-1.126636$

$1-3.632761-1.4694430 .046057$

$8-1.779332-0.0224871 .432250$

$60.802753-0.226141-0.358432$

$80.349203-1.369196-0.526020$

$72.100040-0.003030-0.080009$

$62.952734-1.1554520 .196120$ 
$13.994538-0.8464030 .110854$

$12.775855-1.5431011 .205563$

$12.755107-1.947548-0.525562$

62.6054011 .3045970 .331838

12.4649731 .4642581 .406582

13.6713861 .3485030 .106521

$12.1143622 .105730-0.218432$

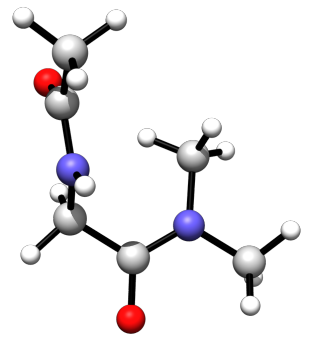

1C'

01

$\#$ imaginary freq. $=0$

$E=-495.675996$

$71.066034-0.736848-0.423457$

$10.967671-0.870462-1.422751$

$60.029739-1.2709820 .438040$

$1-0.011428-2.3582400 .354194$

$10.273002-1.0164051 .471911$

$62.142395-0.0605490 .023311$

$63.0818900 .467784-1.025448$

$14.1016140 .180875-0.760628$

$12.8438770 .105425-2.026230$

$13.0228171 .559971-1.014294$

82.3478120 .1382471 .231698

$6-1.360118-0.7585800 .072729$

$8-2.257559-1.581043-0.193443$

$7-1.5714570 .5644490 .029376$

$6-2.8677471 .059356-0.421103$

$1-3.2672380 .406216-1.196408$

$1-3.5808331 .1072430 .408605$

$1-2.7306952 .061342-0.830181$

$6-0.6276831 .5715110 .505453$

10.1008761 .1388521 .189144

$1-0.1053282 .049753-0.329605$

$1-1.1892562 .3333511 .050873$

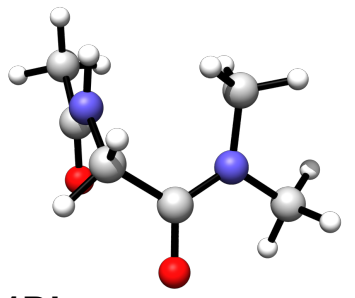

1D'

01

$\#$ imaginary freq. $=0$
$E=-495.676727$

$7-1.270006-0.1147270 .944401$

$1-1.5412780 .6063041 .600419$

$6-0.109316-0.9330411 .234409$

$10.213944-0.7175822 .255400$

$1-0.367118-1.9921521 .173630$

$6-1.846457-0.130777-0.271216$

$6-2.9684190 .840075-0.509526$

$1-3.8525600 .281707-0.826136$

$1-3.2074911 .4328910 .374221$

$1-2.6786021 .505822-1.326762$

$8-1.461418-0.916537-1.153690$

$61.061306-0.7312010 .273535$

$81.619479-1.732972-0.212947$

$71.4642150 .516340-0.008636$

$62.5036830 .714085-1.013476$

$12.3620351 .695040-1.469716$

$13.5012030 .670772-0.563649$

$12.426265-0.053227-1.783197$

60.9974861 .7167580 .680841

10.2081502 .2210720 .114084

10.6359791 .4822341 .681535

11.8458262 .3964980 .785532

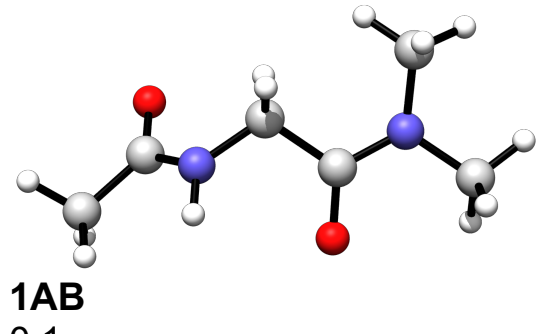

01

\# imaginary freq. $=1$

$\mathrm{E}=-495.676634$

$7-1.389883-0.1118850 .611604$

$1-1.404806-0.9722401 .144980$

$6-0.1262830 .5700530 .476912$

$1-0.2425761 .362866-0.264693$

10.1597691 .0385691 .424918

$6-2.4838490 .208071-0.104564$

$6-3.689617-0.6719170 .084466$

$1-3.968203-1.085615-0.887920$

$1-3.516999-1.4858180 .790111$

$1-4.518201-0.0529910 .437329$

$8-2.5170751 .182833-0.874754$

$60.975194-0.3957910 .051939$

$80.733632-1.605635-0.094951$

$72.2024890 .116555-0.145871$

$63.299954-0.814981-0.390330$

$13.585994-1.3367360 .529621$ 
$13.006424-1.552488-1.137243$

$14.155631-0.251665-0.762130$

62.5725071 .4733030 .248594

$13.3807591 .814803-0.399219$

11.7359142 .1595020 .131777

12.9181621 .4991291 .287870

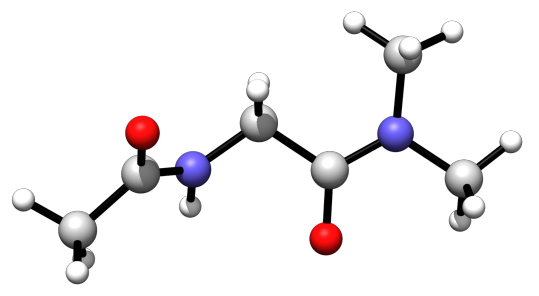

$1 \mathrm{AB}^{\prime}$

01

\# imaginary freq. $=1$

$E=-495.676572$

$7-1.406052-0.160073-0.643231$

$1-1.465985-1.049078-1.123848$

$6-0.1277450 .507720-0.623824$

$10.1211370 .886484-1.620696$

$1-0.2007381 .3642840 .049084$

$6-2.4489040 .2207730 .119039$

$6-3.676197-0.6472770 .059852$

$1-4.521876-0.030927-0.254551$

$1-3.567852-1.492380-0.621226$

$1-3.883975-1.0161181 .067453$

$8-2.4203111 .2403130 .828894$

$60.979439-0.431990-0.157531$

$80.734558-1.6234170 .095371$

$72.2206810 .076585-0.066896$

$63.265302-0.7732350 .497667$

$14.231813-0.3089270 .303385$

$13.135229-0.8917391 .578940$

$13.241676-1.7578420 .030391$

$62.4985951 .509667-0.126656$

12.4148371 .9679220 .864811

$13.5156581 .650329-0.494758$

$11.8207502 .013291-0.813498$

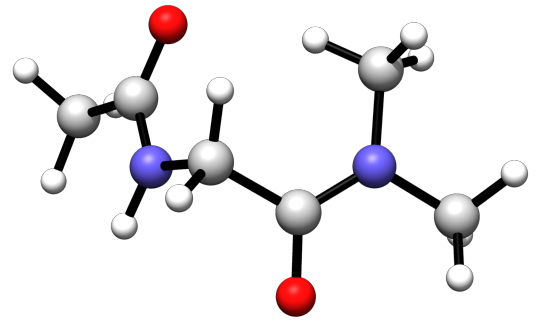

1BC

01

\# imaginary freq. $=1$

$E=-495.673549$

$7-1.340498-0.7992080 .313080$
$1-1.519390-1.732186-0.036928$

$6-0.149036-0.5895141 .115606$

$1-0.2356880 .3638181 .630766$

$1-0.083007-1.3816211 .864713$

$6-2.1726500 .195218-0.051685$

$6-3.338780-0.190570-0.920505$

$1-3.2530250 .346938-1.868386$

$1-3.386721-1.262791-1.114777$

$1-4.2590860 .134596-0.429721$

$8-2.0010511 .3712450 .309013$

$61.105908-0.7216680 .259659$

$81.430269-1.863475-0.126140$

$71.8317890 .361966-0.042835$

$63.0739630 .186037-0.789602$

$13.7266231 .033229-0.574877$

$13.567818-0.735361-0.483063$

$12.8812720 .147980-1.866866$

61.4289371 .7475910 .191228

10.3647141 .8188110 .404115

12.0061862 .1842531 .011330

$11.6274692 .318295-0.719202$

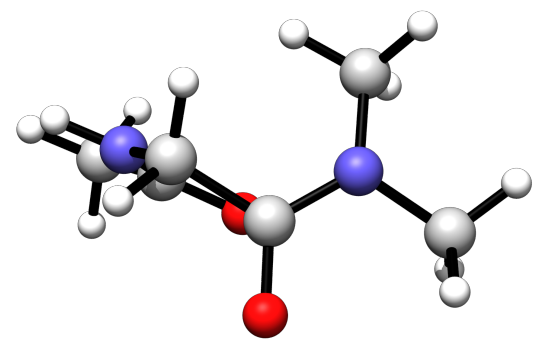

1BD'

01

\# imaginary freq. $=1$

$E=-495.671252$

71.4880280 .7217550 .638470

12.2523791 .1268811 .164156

60.1696181 .2782590 .922327

$1-0.1044421 .0646401 .957613$

10.2236722 .3625970 .808916

$61.790069-0.246323-0.245523$

$63.243127-0.631051-0.337241$

$13.563202-0.520998-1.375994$

$13.886386-0.0317460 .307944$

$13.336285-1.685507-0.065022$

$80.937065-0.803393-0.955642$

$6-0.9217820 .822272-0.040506$

$8-1.1438831 .497214-1.059741$

$7-1.695232-0.2244250 .309466$

$6-2.691246-0.666398-0.659706$

$1-3.361224-1.373420-0.169215$

$1-3.2694400 .181953-1.024026$

$1-2.206101-1.161646-1.510165$ 
$6-1.232904-1.2775351 .208596$ $1-0.887331-2.1343610 .620052$ $1-0.411638-0.9386181 .836790$ $1-2.058067-1.5969901 .848786$

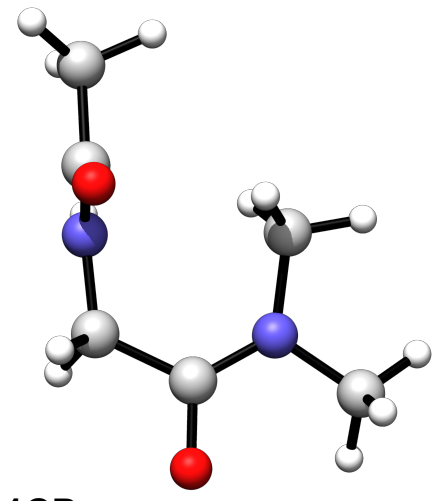

1CD

01

\# imaginary freq. $=1$

$E=-495.670211$

$7-1.206224-0.612285-0.735799$

$1-1.348912-0.331426-1.697599$

$6-0.034653-1.384046-0.405361$

$1-0.256865-2.0106320 .461321$

$10.182331-2.053021-1.240974$

$6-2.048928-0.1360990 .200100$

$6-3.1737340 .730716-0.290229$

$1-2.9296041 .768249-0.038748$

$1-3.3239930 .652852-1.367775$

$1-4.0906500 .4600650 .236271$

$8-1.882651-0.3685501 .408726$

$61.303212-0.711562-0.092395$

$82.230865-1.5103290 .151131$

$71.5176890 .613889-0.110185$

62.8799691 .0521650 .206848

$12.9905832 .091724-0.096546$

13.0828050 .9653881 .279443

$13.6038380 .444177-0.335308$

$60.4791201 .651984-0.161107$

$1-0.0622331 .638808-1.106888$

$1-0.2200311 .5587690 .672907$

$10.9768452 .615908-0.070207$

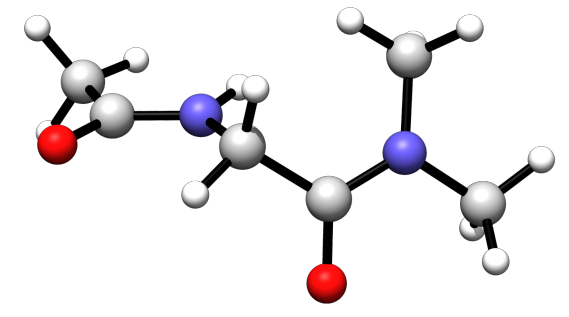

1CD'

01
\# imaginary freq. $=1$

$E=-495.672722$

$7-1.1016880 .2877880 .030929$

$1-0.7588921 .130901-0.414890$

$6-0.188227-0.5201630 .824522$

$1-0.035683-0.0906721 .818998$

$1-0.630353-1.5078340 .943273$

$6-2.4227620 .027869-0.035092$

$6-3.2507870 .964115-0.871678$

$1-4.0331761 .391836-0.240219$

$1-2.6622861 .766588-1.317715$

$1-3.7335530 .383705-1.661868$

$8-2.937320-0.9356100 .554987$

$61.128372-0.7146240 .084674$

$81.328482-1.775945-0.535180$

72.0463740 .2641540 .122655

$63.2680010 .087162-0.657243$

$13.681987-0.906770-0.486201$

$13.0700760 .209782-1.727270$

$13.9912850 .837760-0.338934$

61.8120261 .6107360 .635905

$11.6892302 .313438-0.194825$

10.9267891 .6494231 .267253

12.6727461 .9201171 .232327

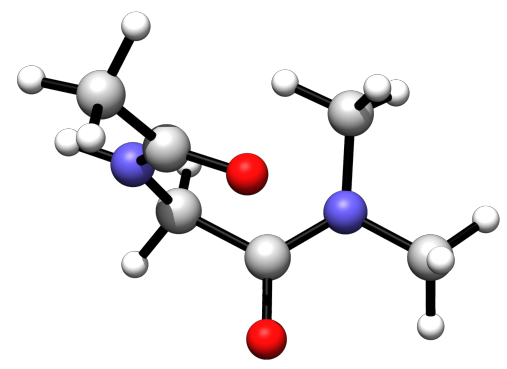

1DB'

01

\# imaginary freq. $=1$

$E=-495.671252$

$7-1.4880000 .7214610 .638664$

$1-2.2525991 .1280231 .162882$

$6-0.1695851 .2782490 .922320$

$1-0.2238702 .3625530 .808717$

10.1045521 .0648691 .957629

$6-1.790219-0.246551-0.245361$

$6-3.243416-0.630741-0.337302$

$1-3.337353-1.684324-0.061923$

$1-3.886950-0.0292090 .305539$

$1-3.562473-0.523728-1.376671$

$8-0.937279-0.804256-0.955031$

$60.9217330 .822294-0.040662$

$81.1434091 .497037-1.060116$

$71.695488-0.2241760 .309337$ 
$62.691378-0.666124-0.659970$ $13.2693080 .182277-1.024593$ $13.361627-1.372901-0.169497$ $12.206156-1.161644-1.510225$ $61.233570-1.2771881 .208779$ $10.412983-0.9380131 .837726$ $10.887203-2.1338730 .620509$ $12.059185-1.5969111 .848248$

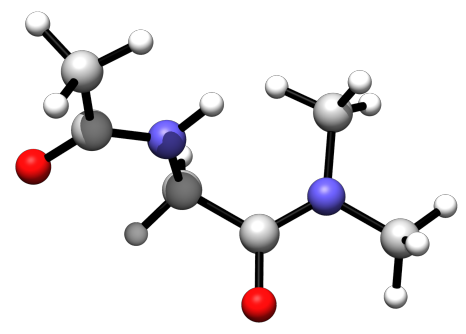

1DC'

01

\# imaginary freq. $=1$

$E=-495.672722$

71.1016980 .2878480 .031012

$10.7589241 .131012-0.414726$

$60.188230-0.5201290 .824566$

$10.630373-1.5077920 .943308$

$10.035657-0.0906611 .819048$

$62.4227560 .027872-0.035098$

$63.2507800 .964150-0.871650$

$13.7334040 .383796-1.661968$

$12.6623061 .766735-1.317521$

$14.0332791 .391714-0.240222$

$82.937302-0.9356830 .554868$

$6-1.128355-0.7146180 .084697$

$8-1.328438-1.775965-0.535123$

$7-2.0463680 .2641510 .122616$

$6-3.2679750 .087122-0.657307$

1 -3.070033 $0.209762-1.727328$

$1-3.681926-0.906828-0.486284$

$1-3.9912960 .837688-0.339007$

$6-1.8120851 .6107340 .635892$

$1-0.9269261 .6494251 .267349$

$1-1.6892002 .313442-0.194819$

$1-2.6728801 .9201051 .232210$

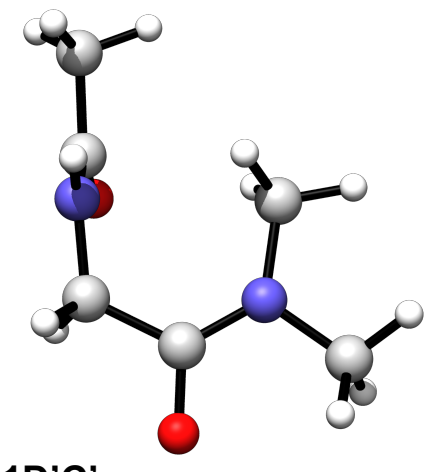

1D'C

01

\# imaginary freq. $=1$

$E=-495.670211$

$71.206640-0.611506-0.736277$

$11.349646-0.329766-1.697770$

$60.034964-1.383584-0.406938$

$1-0.182126-2.051166-1.243656$

$10.257160-2.0116800 .458639$

$62.048972-0.1360750 .200361$

$63.1737840 .731359-0.288895$

$12.9273271 .769110-0.040539$

14.0897060 .4633570 .240638

$13.3269250 .651287-1.365879$

$81.882223-0.3695831 .408715$

$6-1.302947-0.711708-0.092680$

$8-2.229815-1.5110630 .151870$

$7-1.5181810 .613635-0.110765$

$6-2.8800591 .0519020 .207881$

$1-3.0796940 .9705211 .281514$

$1-2.9927842 .089763-0.100596$

$1-3.6046720 .440314-0.329109$

$6-0.4800611 .652146-0.161771$

10.2188101 .5596800 .672579

$10.0616041 .638739-1.107342$

$1-0.9783042 .615876-0.071610$

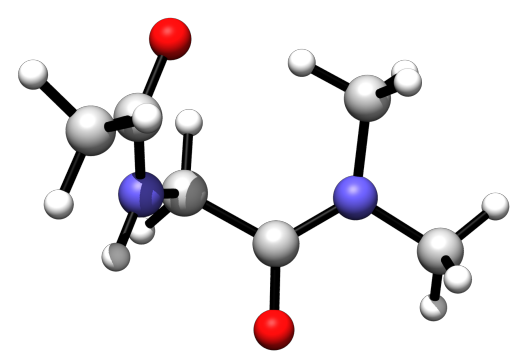

1B'C'

01

\# imaginary freq. $=1$

$E=-495.673504$

$7-1.324266-0.828783-0.292387$

$1-1.490016-1.7398220 .116756$ 
$6-0.149470-0.664199-1.130128$ $1-0.089693-1.505716-1.822952$ $1-0.2553460 .250192-1.707498$ $6-2.1420130 .1863090 .047942$ $6-3.278229-0.1523670 .974228$ $1-3.344972-1.2196501 .189705$ $1-3.1342670 .3972511 .908268$ $1-4.2107950 .1903140 .520468$ $8-1.9805661 .341232-0.378406$ $61.114029-0.728314-0.279348$ $81.474434-1.8482720 .137500$ 71.8068270 .3818600 .008834 62.9945390 .2415680 .848042 13.5747371 .1616100 .778595 12.7161050 .0704521 .893075 $13.602931-0.5949440 .503386$ $61.3752781 .756128-0.231209$ $10.4466891 .792844-0.792701$ 11.2177172 .2564510 .729267 $12.1532292 .291049-0.781407$

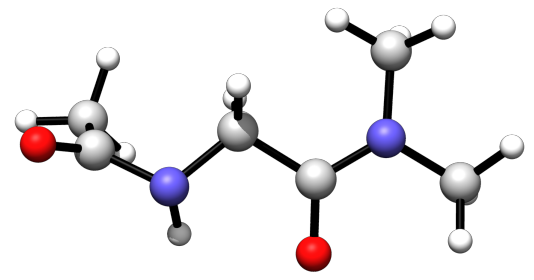

1A/1A-E

01

\# imaginary freq. $=1$

$E=-495.645038$

$71.354784-0.679143-0.327672$ $11.216003-1.3533710 .430928$ $60.2523210 .285634-0.233432$ 10.3537030 .9866360 .608739 $10.2200870 .874972-1.152049$ $62.629768-0.053507-0.110515$ 62.8719720 .6761931 .181562 12.2995270 .2383912 .001951 13.9385740 .6717681 .407701 12.5508231 .7165651 .059355 $83.482100-0.120874-0.975513$ $6-1.052765-0.464037-0.023963$ $8-1.034601-1.6641860 .297666$ $7-2.2025330 .218121-0.177344$ $6-3.447533-0.4450790 .197876$ $1-4.2780780 .097143-0.254914$ $1-3.449467-1.471857-0.165480$ $1-3.575706-0.4507551 .286289$ $6-2.2623621 .674992-0.251387$ $1-3.1107461 .959831-0.875770$
$1-2.3989962 .1054270 .746848$ $1-1.3598822 .087696-0.696556$

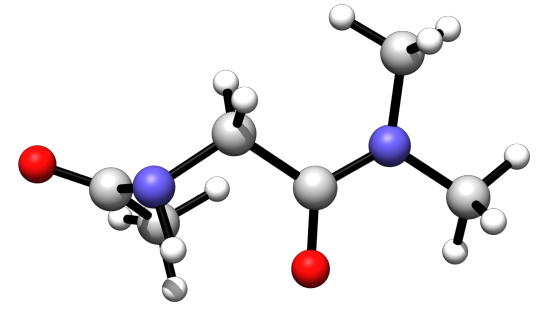

1B/1B-E

01

$\#$ imaginary freq. $=1$

$E=-495.647076$

$71.524623-0.3942620 .963445$

11.3206140 .4664001 .480914

$60.240218-0.8908710 .453387$

$10.390487-1.375912-0.514543$ $1-0.140261-1.6599591 .133773$

$62.427801-0.032532-0.097537$

$62.0051741 .026386-1.073528$

$11.0529390 .760773-1.542997$

$11.8477821 .962481-0.528086$

$12.7691061 .161359-1.838650$

$83.511237-0.580441-0.165291$

$6-0.8212930 .2014230 .353131$

$8-0.5936831 .3292850 .825128$

$7-1.993670-0.104377-0.228881$

$6-3.0685940 .881232-0.159018$ $1-3.5007210 .9200010 .847074$

$1-2.6898611 .869128-0.419895$

$1-3.8442540 .595871-0.869525$

$6-2.402061-1.474002-0.529776$

$1-3.059009-1.459308-1.400876$

$1-1.544886-2.101869-0.763283$

$1-2.946511-1.9090750 .315497$

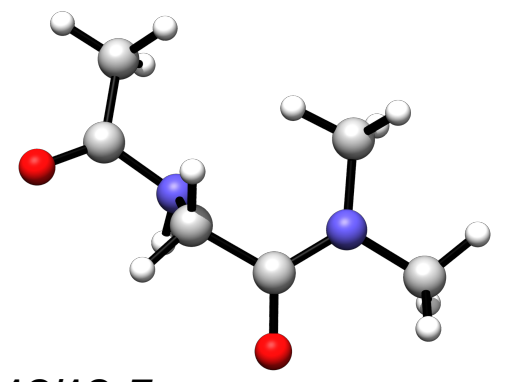

1C/1C-E

01

\# imaginary freq. $=1$

$E=-495.642472$

$7-1.049921-0.198671-0.557983$

$1-1.042562-0.894090-1.308275$

$6-0.239793-0.7396150 .554470$ 
$1-0.403772-0.1223571 .441186$

$1-0.500860-1.7718440 .802635$

$6-2.426085-0.063095-0.139827$

$6-2.9841131 .314055-0.270185$

$1-4.0189811 .3523720 .067729$

$1-2.3607051 .9959030 .318613$

$1-2.9059841 .632311-1.314322$

$8-3.054275-1.0058360 .303069$

$61.222563-0.7384230 .143651$

$81.757082-1.810018-0.206715$

71.9075400 .4143720 .152840

$63.2855930 .394185-0.328585$

$13.845354-0.4064010 .156944$

$13.3193950 .240938-1.412213$

$13.7483021 .351303-0.088581$

61.3294131 .7336890 .385821

$11.3222222 .306151-0.547198$

10.3101651 .6604210 .755430

11.9361662 .2674391 .121162

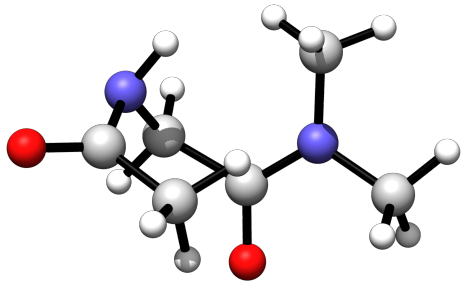

1D/1D-E

01

\# imaginary freq. $=1$

$E=-495.642640$

71.3727660 .9667580 .470854

$10.9767311 .650412-0.174950$

60.3362930 .4464061 .377285

$10.815301-0.2319412 .084582$

$1-0.0516831 .2951991 .946307$

$62.059483-0.021010-0.320427$

$61.272160-0.910468-1.240448$

$11.939085-1.358390-1.977167$

$10.810051-1.712061-0.653332$

$10.472995-0.350478-1.735207$

$83.269368-0.100962-0.235112$

$6-0.837113-0.3348470 .784122$

$8-1.006370-1.5238211 .118469$

$7-1.6772570 .270930-0.071227$

$6-2.642551-0.573065-0.771444$

$1-3.2685330 .060723-1.398036$

$1-2.126376-1.306066-1.401176$

$1-3.273050-1.107335-0.058491$

$6-1.4977171 .631655-0.565816$

$1-1.0424062 .2644200 .196413$

$1-0.8888751 .648916-1.477891$
$1-2.4791282 .049022-0.794931$

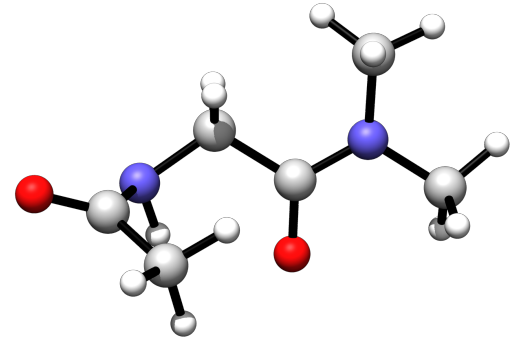

1B'/1B'-E

01

\# imaginary freq. $=1$

$E=-495.646898$

$7-1.5438750 .279594-1.006248$

$1-1.342170-0.627826-1.437995$

$6-0.2489590 .848759-0.611163$

$10.0811541 .555787-1.379117$

$1-0.3611091 .4219390 .313229$

$6-2.3898270 .0155890 .128242$

$6-1.901515-0.9285061 .188101$

$1-2.634699-1.0073611 .990237$

$1-1.731151-1.9096460 .733464$

$1-0.943518-0.5893091 .595002$

$8-3.4798140 .5510140 .191167$

$60.837983-0.211723-0.452287$

$80.612937-1.386477-0.794384$

72.0319830 .1814150 .018142

$63.044211-0.8395010 .273340$

$14.010966-0.3495290 .385394$

$12.817515-1.3992451 .187298$

$13.090889-1.535992-0.563834$

62.2514331 .5070260 .592539

11.9276341 .5475761 .638116

13.3176101 .7290860 .546241

11.7251862 .2713050 .021819

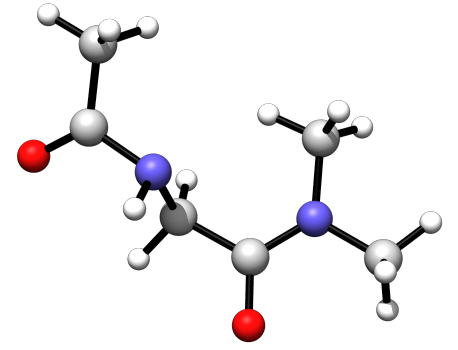

$1 C^{\prime} / 1 C^{\prime}-E$

01

\# imaginary freq. $=1$

$E=-495.642472$

$71.049384-0.196794-0.557493$

$11.040727-0.889593-1.310189$

$60.239655-0.7402920 .553911$

$10.501154-1.7728740 .800121$ 
$10.403516-0.1246131 .441789$ $62.425930-0.063389-0.140061$ $62.9847101 .313741-0.267518$ $12.9083361 .633184-1.311456$ 12.3606351 .9951970 .320986 14.0191271 .3512110 .071872 $83.053879-1.0077240 .299753$ $6-1.222923-0.7384780 .143913$ $8-1.758945-1.810072-0.204204$ $7-1.9067740 .4149480 .151552$ $6-3.2850770 .394746-0.329274$ $1-3.847009-0.4004850 .162672$ $1-3.7450681 .354999-0.096817$ $1-3.3196080 .232889-1.411587$ $6-1.3281481 .7338660 .385642$ $1-0.3102701 .6597860 .758878$ $1-1.3172662 .306042-0.547509$ $1-1.9368972 .2683821 .118764$

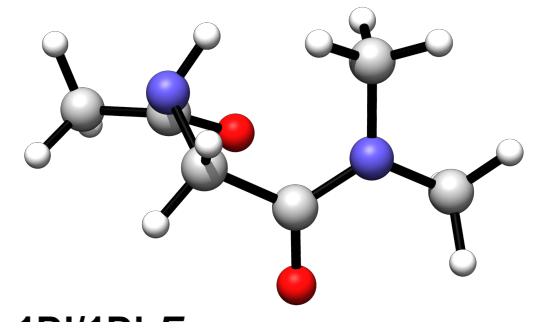

1D'/1D'-E

01

\# imaginary freq. $=1$

$E=-495.644272$

$71.314091-0.9189940 .563978$ $10.906241-1.7045730 .054955$ $60.289620-0.2800111 .408798$ $1-0.131083-1.0489872 .059874$ 10.7848810 .4593472 .040555 $61.849980-0.019006-0.428874$ $63.3264220 .184323-0.367092$ 13.5834230 .5757060 .622794 $13.823278-0.785792-0.465915$ $13.6601180 .867141-1.147473$ $81.1313970 .527084-1.245324$ $6-0.8300180 .4726090 .690176$ $8-0.8848551 .7133040 .787238$ $7-1.757078-0.2202790 .001492$ $6-2.6726490 .537910-0.846167$ $1-3.465043-0.128263-1.186477$ $1-3.1143601 .360878-0.284775$ $1-2.1459410 .945543-1.717232$ $6-1.622661-1.639417-0.308158$ $1-1.041850-1.792257-1.225963$ $1-1.155907-2.1767550 .517262$ $1-2.619341-2.058632-0.453294$

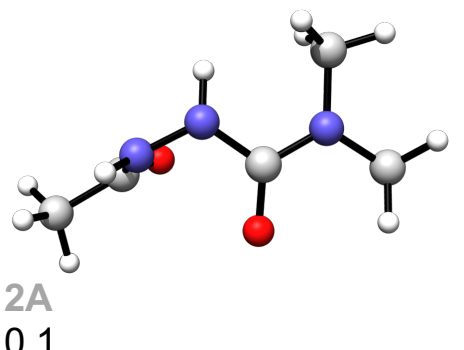

$\#$ imaginary freq. $=0$

$E=-511.696474$

$71.4232110 .527552-0.778763$

$11.8292630 .478218-1.704828$

62.1120840 .0995290 .308959

$63.543314-0.2791670 .064069$

$13.657870-1.3415650 .294398$

$13.855452-0.095932-0.964896$

14.1764000 .2891720 .748733

81.5857140 .0429741 .424396

$6-0.765110-0.279739-0.438418$

$8-0.361820-1.440638-0.590943$

$7-2.0371700 .033870-0.100092$

$6-2.893302-1.0475560 .379479$

$1-2.723149-1.2451751 .444102$

$1-3.934087-0.7570740 .233879$

$1-2.696782-1.955166-0.188099$

$6-2.4119821 .3939020 .282493$

$1-2.1720882 .104523-0.511535$

$1-3.4906951 .4178990 .432723$

$1-1.9233901 .7044131 .213748$

$70.0758290 .808621-0.699459$

$1-0.1130551 .669893-0.197149$

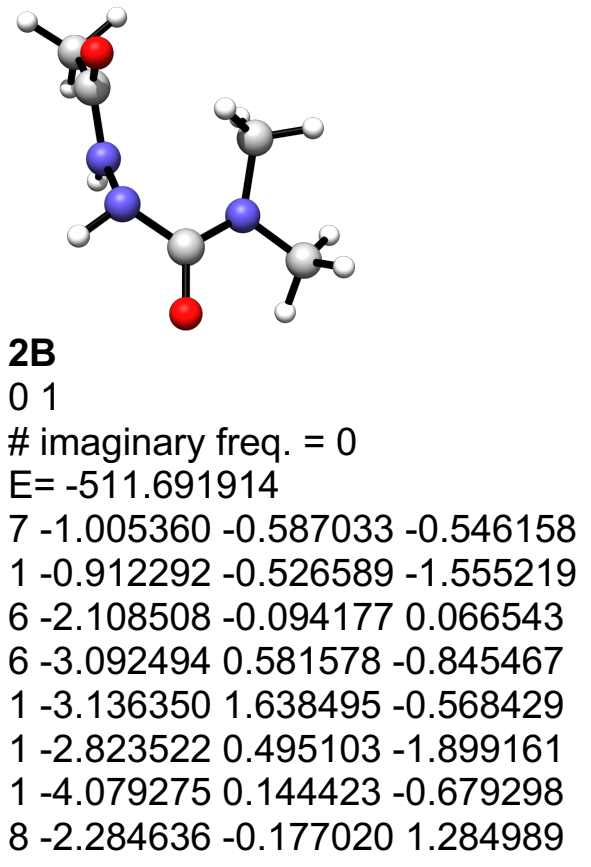


$61.313552-0.7499550 .012312$

$82.236495-1.579821-0.041833$

$71.5042200 .581626-0.047049$

$62.8598111 .060979-0.293892$

$12.7958552 .067376-0.710092$

13.4446451 .0932990 .632045

$13.3599700 .411126-1.010971$

60.5661601 .5521520 .518665

$1-0.0296812 .043921-0.255737$

$1-0.0962461 .0707221 .238748$

11.1484262 .3084641 .049460

$70.002416-1.2180620 .162777$

$1-0.026452-2.2307810 .077467$

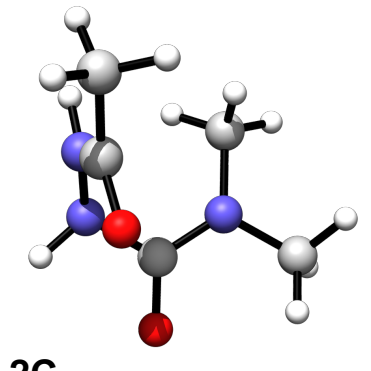

2C

$E=-511.694023$

$7-1.189406-0.175741-0.917302$

$1-1.4718740 .450609-1.661186$

$6-1.816586-0.1574370 .283986$

$6-2.9893700 .7729370 .388349$

$1-2.7690751 .5140821 .160929$

$1-3.2061981 .281205-0.552203$

$1-3.8621880 .1980080 .705679$

$8-1.429757-0.8612521 .222406$

$61.058968-0.718368-0.284007$

$81.715814-1.7059870 .086478$

71.3790400 .5478960 .031812

62.4614380 .7678540 .984809

12.2769421 .7092581 .505152

$12.485168-0.0418461 .712966$

13.4302540 .8248840 .476744

$60.8993801 .723885-0.691795$

$10.6162271 .461568-1.711716$

$10.0517512 .196352-0.185675$

$11.7220952 .439974-0.745325$

$7-0.046396-0.929831-1.126427$

$1-0.247192-1.925679-1.181066$

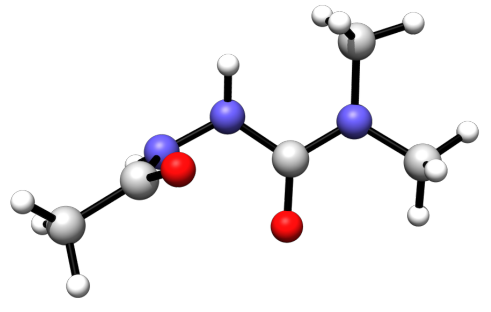

\section{A'}

01

\# imaginary freq. $=0$

$\mathrm{E}=-511.696474$

$7-1.4231810 .527877-0.778468$

$1-1.8289390 .478807-1.704673$

$6-2.1122540 .0992630 .308896$

$6-3.543556-0.2789270 .063664$

$1-4.1766190 .2907470 .747261$

$1-3.855009-0.096654-0.965678$

$1-3.659014-1.3409330 .295267$

$8-1.5860350 .0420931 .424383$

$60.765137-0.279525-0.438295$

$80.361870-1.440412-0.591465$

$72.0371280 .033850-0.099717$

$62.893397-1.0477310 .379189$

$13.934145-0.7570830 .233648$

$12.723386-1.2459291 .443726$

$12.696910-1.955074-0.188827$

62.4124141 .3938440 .282390

11.9252081 .7042881 .214393

13.4913641 .4179470 .430948

$12.1712572 .104483-0.511226$

$7-0.0758090 .808954-0.698672$

$10.1128351 .669652-0.195255$

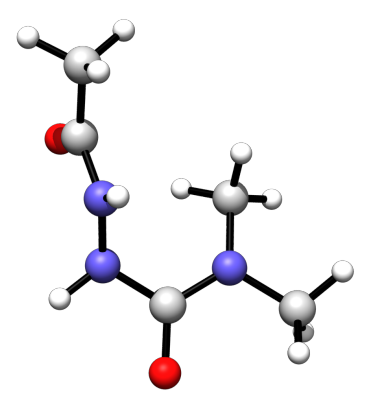

2B'

01

$\#$ imaginary freq. $=0$

$\mathrm{E}=-511.691914$

$71.005312-0.587011-0.546152$

$10.912190-0.526468-1.555205$

$62.108530-0.0941990 .066494$

$63.0924170 .581656-0.845548$

$14.0792360 .144563-0.679444$

$12.8233880 .495181-1.899226$

$13.1362241 .638571-0.568492$

$82.284729-0.1770791 .284924$

$6-1.313565-0.7500060 .012432$

$8-2.236452-1.579967-0.042023$

$7-1.5043570 .581525-0.046507$ 
$6-2.8597101 .061122-0.294074$ $1-2.7953212 .067044-0.711378$ $1-3.3600190 .410635-1.010463$ $1-3.4446231 .0947260 .631764$ $6-0.5661971 .5521720 .518729$ 10.0965811 .0708651 .238544 $10.0292112 .044019-0.255960$ $1-1.1483442 .3084111 .049779$ $7-0.002391-1.2180650 .162829$ $10.026454-2.2307870 .077488$

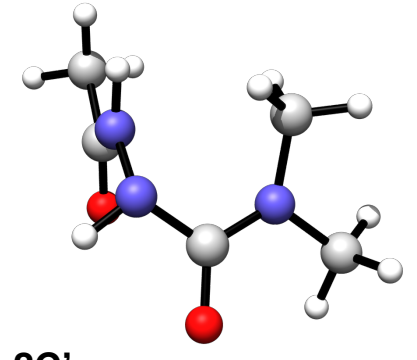

2C'

01

$\#$ imaginary freq. $=0$

$\mathrm{E}=-511.694023$

$71.189475-0.175757-0.917413$ $11.4715920 .451018-1.661065$ $61.816612-0.1575250 .283904$ 62.9889340 .7733810 .388586 13.8614110 .1994110 .708535 $13.2071241 .280034-0.552521$ 12.7669761 .5159291 .159359 $81.429942-0.8617231 .222103$ $6-1.058903-0.718351-0.283956$ $8-1.715546-1.7060900 .086610$ $7-1.3790980 .5478550 .031758$ $6-0.8992621 .723886-0.691671$ $1-1.7218082 .440190-0.744830$ $1-0.0513992 .196014-0.185634$ $1-0.6164541 .461769-1.711745$ $6-2.4615290 .7679090 .984720$ $1-2.486088-0.0423791 .712176$ $1-2.2763881 .7087491 .505873$ $1-3.4301530 .8261700 .476436$ $70.046409-0.929874-1.126577$ $10.247401-1.925764-1.180162$

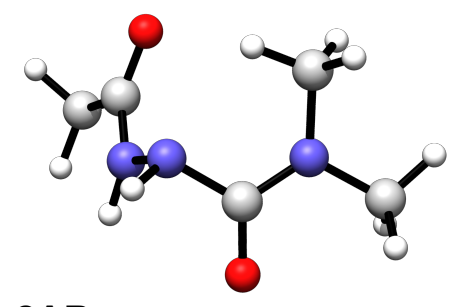

$2 A B$
01

$\#$ imaginary freq. $=1$

$\mathrm{E}=-511.679807$

$71.291814-0.729680-0.420063$

$11.439851-1.695628-0.142588$

62.0954230 .2433020 .066572

$63.268306-0.2447210 .871297$

13.1665410 .1365631 .890527

$13.342143-1.3326020 .896749$

14.1797400 .1750890 .440073

$81.8938981 .446160-0.133799$

$6-1.026692-0.707923-0.296837$

$8-1.275068-1.8728670 .047885$

$7-1.7704590 .3358680 .066180$

$6-2.9153000 .0679390 .934474$

$1-3.4129131 .0122331 .150004$

$1-3.619310-0.6072860 .441921$

$1-2.586037-0.3889911 .870995$

$6-1.5277171 .727781-0.305673$

$1-0.7003731 .785718-1.005757$

$1-2.4308302 .134897-0.766956$

$1-1.2939282 .3112680 .589146$

$70.124499-0.409170-1.124926$

$10.079374-1.014991-1.944130$

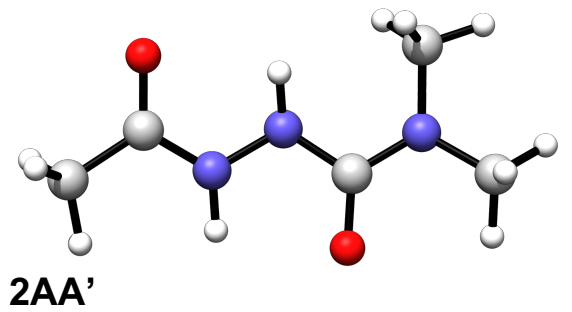

01

$\#$ imaginary freq. $=1$

$\mathrm{E}=-511.690634$

$7-1.318100-0.456623-0.170523$

$1-1.252758-1.465453-0.250233$

$6-2.4550360 .2156060 .044375$

$6-3.702983-0.6088180 .155819$

$1-4.398278-0.287800-0.623701$

$1-3.509568-1.6773140 .053186$

$1-4.163696-0.4112611 .126524$

$8-2.4525051 .4567280 .146103$

$61.021293-0.511167-0.134271$

$80.944642-1.749056-0.050599$

$72.1861360 .178750-0.139208$

$63.385390-0.5152410 .319991$

$14.257130-0.035593-0.126484$

$13.473433-0.4699951 .411761$

$13.354052-1.5563710 .005163$

$62.1948991 .635325-0.035716$ 
11.8648501 .9756630 .953255

$13.2153681 .980106-0.200388$ $11.5625652 .081767-0.806016$ $7-0.1264340 .240092-0.329304$ $1-0.1927871 .1751140 .064961$

2AC'

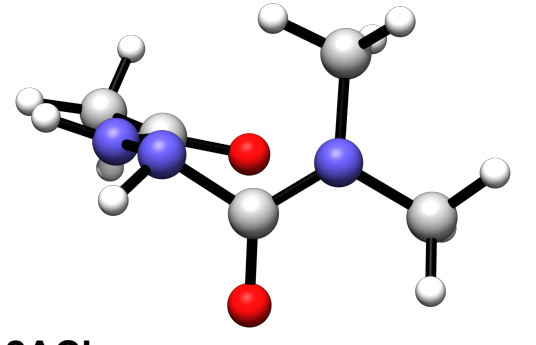

01

\# imaginary freq. $=1$

$E=-511.673279$

$7-1.4647900 .690058-0.649840$

$1-2.1777951 .067617-1.262219$

$6-1.773756-0.2796760 .221409$

$6-3.240984-0.5789260 .360109$

$1-3.8658680 .059663-0.265366$

$1-3.403715-1.6250180 .088878$

$1-3.520752-0.4505011 .408220$

$8-0.911597-0.8817760 .884469$ $60.8436600 .805495-0.006968$ 80.9301691 .5756050 .954160

$71.714666-0.180932-0.259870$

$61.418959-1.280201-1.173632$

$12.339459-1.576804-1.680512$

$11.031858-2.134910-0.608126$

$10.682129-0.971367-1.910490$

$62.664763-0.5028050 .798904$

$12.156777-1.0236661 .619916$

$13.436194-1.1526080 .385052$

13.1265370 .4060891 .182306

$7-0.1446131 .035836-1.050411$

$1-0.1660942 .052807-1.144776$

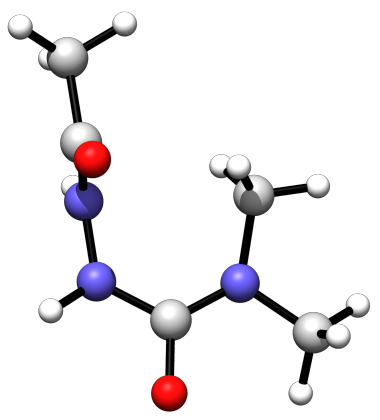

2BC

01

\# imaginary freq. $=1$

$E=-511.687583$
$7-1.139945-0.486942-0.778928$

$1-1.339111-0.266023-1.747580$

$6-2.004757-0.1377970 .207141$

$6-3.1854920 .674742-0.234769$

$1-3.0475071 .6929800 .141861$

$1-3.2899020 .705432-1.320065$

$1-4.0893050 .2617710 .216655$

$8-1.798158-0.4391511 .385201$

$61.222855-0.727507-0.128984$

$82.074527-1.5798910 .187683$

$71.5150340 .596457-0.194298$

62.8768110 .9524860 .213338

$13.0989581 .946997-0.172840$

12.9798110 .9601621 .304543

$13.5906000 .245715-0.205355$

$60.5264681 .678516-0.064129$

$1-0.0827251 .788794-0.960463$

$1-0.1159211 .5352110 .809228$

11.0821612 .6041330 .079858

$7-0.010497-1.221483-0.520054$

$1-0.165464-2.181696-0.231526$

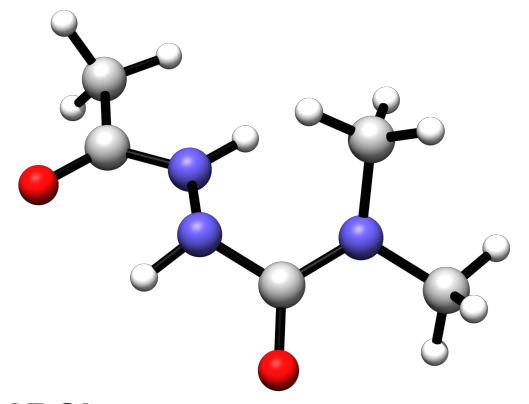

2BC'

01

\# imaginary freq. $=1$

$E=-511.682391$

$7-1.0306500 .226481-0.043385$

$1-0.6475961 .113565-0.345421$

$6-2.348553-0.020176-0.048977$

$6-3.2230491 .077216-0.580041$

$1-3.9252791 .3677020 .205115$

$1-2.6528261 .950673-0.899724$

1 -3.796026 $0.685342-1.423598$

$8-2.797191-1.1060820 .358294$

$61.166257-0.7574520 .032295$

$81.693737-1.847623-0.234443$

$71.8099620 .425280-0.051988$

61.4637241 .5877340 .768515

12.3549231 .8962311 .321315

11.1306782 .4261220 .148974

10.6907851 .3335961 .493571

$63.1692990 .417409-0.582169$

$13.239791-0.275450-1.419701$ 
$13.4041501 .424180-0.930824$ 13.8909230 .1280920 .189848 $7-0.156778-0.7365640 .486843$ $1-0.585698-1.6551880 .391607$

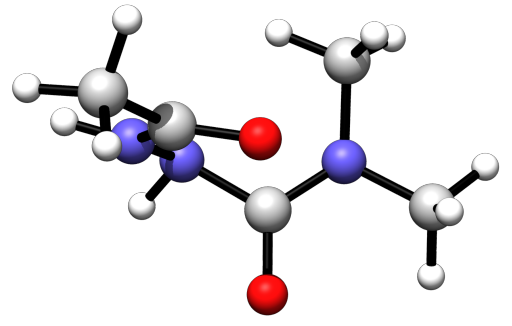

2CA'

01

\# imaginary freq. $=1$

$\mathrm{E}=-511.673279$

$7-1.4649320 .6905830 .649440$

$1-2.1780951 .0681541 .261644$

$6-1.773530-0.279974-0.221112$

$6-3.240760-0.578973-0.360592$

$1-3.404122-1.624579-0.087879$

$1-3.8659050 .0607900 .263435$

1 -3.519639 -0.451970 -1.409104

$8-0.910981-0.882736-0.883056$

60.8432770 .8054630 .006868

$80.9290061 .575248-0.954558$

$71.714833-0.1804650 .259875$

$62.664831-0.502078-0.799118$

$13.436877-1.151183-0.385332$

$12.156921-1.023623-1.619764$

$13.1257680 .407015-1.183006$

$61.419304-1.2800091 .173379$

$10.682893-0.9712361 .910679$

$11.031715-2.1344320 .607763$

$12.339969-1.5770021 .679709$

$7-0.1446921 .0354551 .050744$

$1-0.1657792 .0523811 .145806$

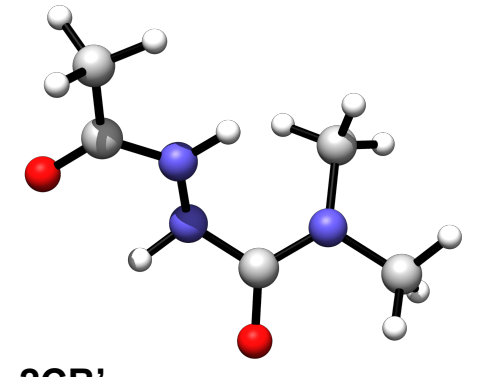

2CB'

01

\# imaginary freq. $=1$

$E=-511.682391$

$71.0306970 .226594-0.043072$

$10.6476801 .113720-0.345018$
$62.348583-0.020167-0.049047$

$63.2230041 .077348-0.579992$

$13.7964560 .685448-1.423209$

$12.6526831 .950590-0.900089$

13.9248061 .3682030 .205416

$82.797301-1.1061570 .357910$

$6-1.166192-0.7574540 .032359$

$8-1.693585-1.847683-0.234288$

$7-1.8099300 .425263-0.052175$

$6-3.1692140 .417285-0.582485$

$1-3.4038591 .423879-0.931809$

$1-3.239744-0.276101-1.419572$

$1-3.8909540 .1286070 .189662$

$6-1.4641101 .5876570 .768602$

$1-0.6910471 .3336571 .493573$

$1-1.1314032 .4263110 .149242$

$1-2.3554141 .8956881 .321503$

$70.156784-0.7364610 .487077$

$10.585772-1.6550630 .391888$

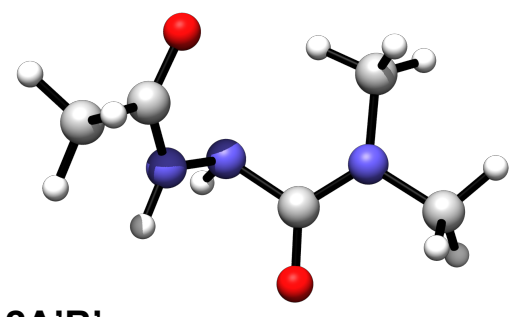

2A'B'

01

\# imaginary freq. $=1$

$E=-511.679807$

$7-1.291788-0.729886-0.419824$

$1-1.439825-1.695736-0.142001$

$6-2.0953190 .2432720 .066591$

$6-3.268424-0.2444540 .871187$

$1-4.1798160 .1744160 .438952$

1 -3.341762 -1.332342 0.897805

1 -3.167474 0.1380591 .890035

$8-1.8936491 .446084-0.133953$

$61.026783-0.708041-0.296734$

$81.275472-1.8728520 .048183$

71.7702930 .3359740 .066163

62.9151950 .0684330 .934474

13.4128621 .0128261 .149464

$12.585969-0.3880001 .871261$

$13.619133-0.6070610 .442197$

$61.5273571 .727764-0.305999$

$10.6998941 .785454-1.005965$

11.2936432 .3114340 .588719

$12.4303652 .134866-0.767512$

$7-0.124467-0.409731-1.124937$

$1-0.079389-1.016113-1.943725$ 


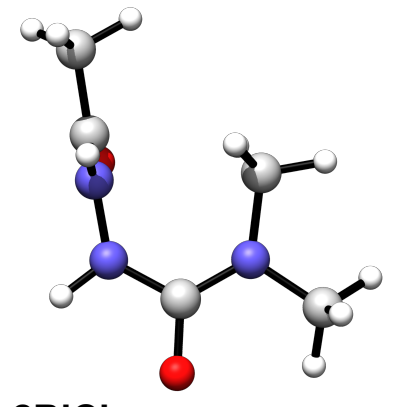

2B'C'

01

\# imaginary freq. $=1$

$E=-511.686309$

$71.124930-0.533840-0.687211$

$11.231958-0.305334-1.669577$

$62.061299-0.1375920 .211760$

$63.1982810 .651318-0.369739$

14.1347220 .2855190 .054699

$13.2349280 .595220-1.458562$

$13.0696721 .695660-0.068815$

$81.958766-0.3713241 .418027$

$6-1.265648-0.733224-0.098859$

$8-2.174450-1.585098-0.015321$

$7-1.4990230 .5869230 .064710$

$6-2.8958680 .9875450 .244560$

$1-3.3898231 .150102-0.719831$

$1-3.4369310 .2230650 .797506$

$1-2.9176121 .9158920 .815543$

$6-0.5482021 .674361-0.194968$ 10.2789011 .6660890 .517772

$1-0.1611781 .650521-1.214958$

$1-1.0890902 .610171-0.061976$

$70.010335-1.233768-0.300180$

$10.017052-2.225172-0.511208$

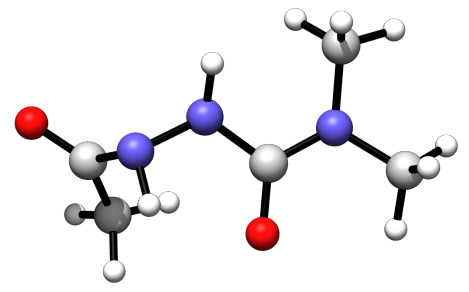

2A/2A-E

01

$\#$ imaginary freq. $=1$

$E=-511.667026$

$71.3882130 .317122-0.977814$

$11.215813-0.573312-1.454522$

62.4088950 .1044560 .039441

$62.260887-1.0775430 .944233$

$11.290816-1.0444821 .448688$

$12.281340-1.9880650 .336313$
$13.066743-1.0950101 .677120$

83.3299450 .8893190 .100343

$6-0.805679-0.291071-0.231200$

$8-0.564746-1.467361-0.550494$

$7-1.9951550 .1204780 .272904$

$6-3.148174-0.7560110 .090309$

$1-2.841303-1.7961990 .178012$

$1-3.879532-0.5348370 .868632$

$1-3.612743-0.597176-0.890087$

$6-2.2897191 .5401840 .444244$

$1-3.2145311 .6266901 .014330$

$1-1.4988932 .0336921 .012065$

$1-2.4233752 .048745-0.518470$

$70.1841170 .677190-0.328409$

$1-0.1034191 .620662-0.559792$

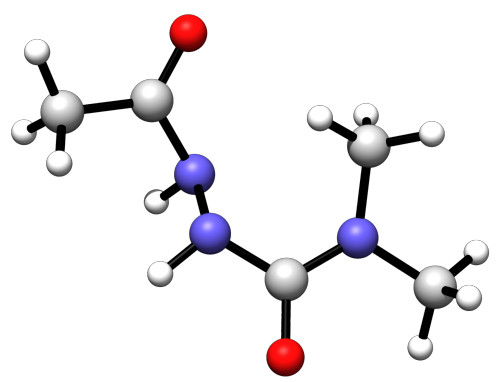

2B/2B-E

01

$\#$ imaginary freq. $=1$

$\mathrm{E}=-511.660725$

$70.984175-0.1491460 .777068$

$11.241564-0.7415081 .569642$

62.1998460 .2951300 .113961

$63.202732-0.751255-0.256416$

$13.562619-1.2222020 .665114$

$12.731702-1.532727-0.859534$

$14.036280-0.307754-0.799533$

$82.3539631 .479674-0.086768$

$6-1.169766-0.804939-0.090112$

$8-1.860307-1.832908-0.223341$

$7-1.7007830 .4233380 .055052$

$6-3.1511300 .5268190 .163975$

$1-3.3907331 .4676610 .662090$

$1-3.6267870 .515181-0.823336$

$1-3.542028-0.2978410 .758829$

$6-0.9958601 .653736-0.299480$

$1-0.6866472 .2140270 .586151$

$1-0.1193091 .433043-0.906210$

$1-1.6770832 .270464-0.891702$

$70.220366-0.928145-0.133514$

$10.479939-1.911744-0.162457$ 


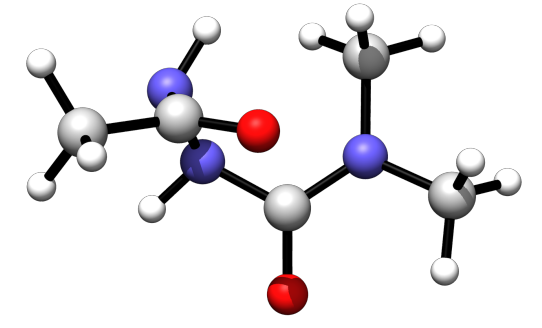

2C/2C-E

01

\# imaginary freq. $=1$

$E=-511.658621$

71.2474390 .3173021 .044869 10.8577341 .2262171 .289001

$61.7449460 .357315-0.326669$

$63.171473-0.045307-0.467576$

$13.484045-0.019226-1.510507$

13.7883200 .6250330 .139324

$13.290221-1.051760-0.052109$

$81.0243200 .674352-1.249452$

$6-0.793204-0.8609080 .294005$

$8-0.964815-1.980374-0.220351$

$7-1.6343990 .1756090 .106511$

$6-2.4743500 .204260-1.083940$

$1-2.474581-0.771583-1.563781$

$1-3.4975360 .470077-0.807253$

$1-2.0915900 .955894-1.784183$

$6-1.5098821 .4533550 .790205$

$1-1.1577191 .3104851 .812597$

$1-0.8461332 .1390080 .248137$

$1-2.5030731 .9047690 .837772$

$70.261021-0.6937011 .213925$

$10.721933-1.5874941 .356141$

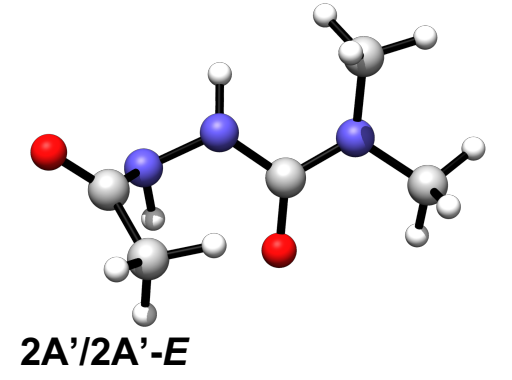

01

\# imaginary freq. $=1$

$E=-511.666711$

7 -1.418392 0.302214 -0.987466

$1-1.257317-0.591191-1.462096$

$6-2.3896190 .0878200 .077458$

$6-2.192003-1.0872980 .981304$

$1-2.965162-1.1071811 .748568$

$1-2.229476-2.0021620 .380873$

$1-1.201298-1.0408581 .442368$
$8-3.3116320 .8674710 .176688$

$60.825615-0.261553-0.363038$

$80.580654-1.455908-0.602851$

$72.0623790 .205684-0.058758$

$63.084640-0.7849940 .269431$

$14.064813-0.3230340 .150080$

$12.979145-1.1396321 .301520$

$13.010432-1.633269-0.408613$

62.2271991 .5364260 .525336

11.6580551 .6441851 .455028

13.2849231 .6793350 .742593

$11.9284522 .320848-0.174528$

$7-0.1895840 .680627-0.396182$

$10.0594461 .638376-0.612591$

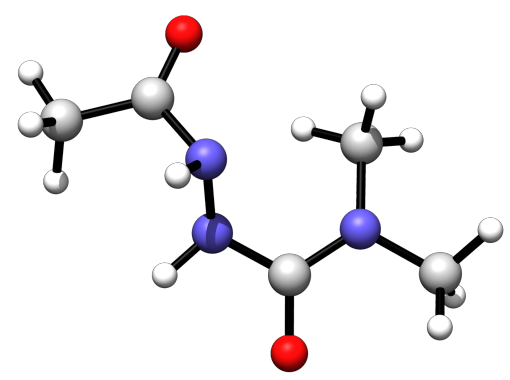

2B'/2B'-E

01

\# imaginary freq. $=1$

$E=-511.660725$

$7-0.984216-0.1491060 .777113$

$1-1.241600-0.7414711 .569684$

$6-2.1998180 .2951420 .113900$

$6-3.202843-0.751209-0.256214$

$1-4.035990-0.307884-0.800088$

$1-2.731727-1.533291-0.858466$

1 -3.563364 -1.221302 0.665505

$8-2.3537831 .479642-0.087204$

$61.169736-0.804935-0.090103$

$81.860230-1.832907-0.223518$

71.7007980 .4233130 .055259

63.1511890 .5266770 .163841

$13.6266080 .515007-0.823583$

13.3909911 .4674880 .661913

$13.542164-0.2980210 .758593$

$60.9959001 .653773-0.299186$

$10.1194401 .433183-0.906090$

10.6865342 .2138990 .586491

$11.6772102 .270613-0.891187$

$7-0.220407-0.928082-0.133496$

$1-0.480051-1.911661-0.162554$ 


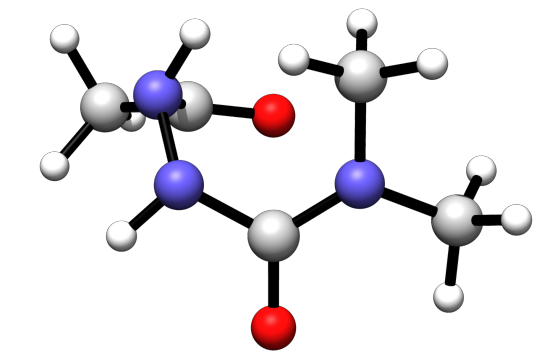

$2 C^{\prime} / 2 C^{\prime}-E$

01

$\#$ imaginary freq. $=1$

$E=-511.658622$

$7-1.247454-0.317180-1.044897$

$1-0.857767-1.226076-1.289131$

$6-1.744952-0.3573330 .326643$

$6-3.1714820 .0452650 .467593$

$1-3.2901951 .0518050 .052324$

$1-3.788322-0.624936-0.139463$

$1-3.4840820 .0189901 .510511$

$8-1.024314-0.6744311 .249396$

$60.7932300 .860934-0.293942$

80.9648991 .9803670 .220469

$71.634369-0.175636-0.106508$

$62.474348-0.2043861 .083919$

$12.091651-0.9561421 .784067$

$13.497551-0.4700980 .807184$

12.4745170 .7713901 .563892

$61.509833-1.453317-0.790315$

$10.846048-2.138998-0.248327$

$11.157709-1.310352-1.812707$

$12.503013-1.904757-0.837887$

$7-0.2610220 .693826-1.213850$

$1-0.7219221 .587637-1.355983$

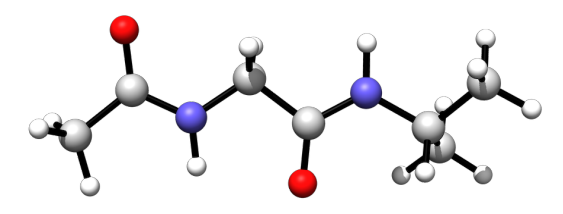

3A

01

\# imaginary freq. $=0$

$E=-534.991135$

$71.922562-0.250751-0.084703$

$11.750187-1.213332-0.352570$

60.7741820 .6013170 .114836

10.7401160 .9752661 .143941

$10.8161591 .470962-0.549705$

63.1738510 .2009960 .075959

$64.289352-0.778887-0.160475$

$14.887337-0.8519220 .751217$
$13.926004-1.768971-0.438370$

$14.930920-0.389325-0.954768$

83.4027011 .3802470 .404405

$6-0.494516-0.185010-0.170571$

$8-0.452402-1.375723-0.517228$

$7-1.6328810 .500757-0.030791$

$6-2.957247-0.098617-0.224821$

$1-2.883295-0.744032-1.105662$

$1-1.5816891 .4625770 .288482$

$6-3.9606741 .013059-0.493219$

$1-3.6872071 .588732-1.381804$

$1-4.9538680 .584706-0.649195$

$1-4.0135781 .6940770 .363697$

$6-3.355274-0.9427370 .982425$

$1-2.607382-1.7140241 .185893$

$1-3.456553-0.3089531 .869794$

$1-4.315352-1.4327310 .795301$

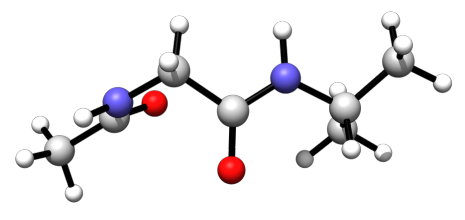

3B

01

\# imaginary freq. $=0$

$E=-534.992746$

$72.150651-0.961021-0.033515$

$12.774764-1.5600410 .489495$

$60.829185-1.430791-0.374000$

$10.665875-1.367825-1.453605$

$10.751511-2.479059-0.077903$

$62.5067500 .313195-0.274914$

63.8769320 .7423200 .167948

13.7662571 .5446780 .901978

$14.451998-0.0740770 .606756$

$14.4116591 .145286-0.695341$

$81.7283991 .097207-0.845256$

$6-0.260303-0.6540350 .359596$

$8-0.075446-0.1935001 .496050$

$7-1.427731-0.561628-0.287449$

$6-2.5878340 .1477210 .259332$

$1-2.635399-0.1023941 .323629$

$1-1.478822-0.894257-1.244200$

$6-3.844187-0.353127-0.437559$

$1-3.978389-1.428294-0.289702$

$1-4.7201710 .163967-0.037890$

$1-3.790065-0.150797-1.513202$

$6-2.4158941 .6565450 .105498$

$1-1.4856681 .9937330 .571417$ 
$1-2.3939451 .926838-0.955686$ $1-3.2515612 .1801550 .579243$

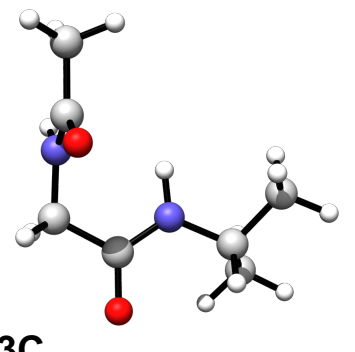

$3 \mathrm{C}$

01

$\#$ imaginary freq. $=0$

$E=-534.992352$

$7-1.767983-0.515287-0.777276$

$1-1.934049-0.386363-1.766975$

$6-0.912076-1.593273-0.337984$

$1-1.374122-2.1245210 .497907$

$1-0.789996-2.299995-1.161341$

$6-2.3824530 .3031690 .102372$

$6-3.2805931 .365574-0.465801$

$1-2.8912782 .342699-0.168650$

$1-3.3542741 .317762-1.552966$

$1-4.2740621 .249902-0.025948$

$8-2.2048710 .1823941 .324583$

$60.483927-1.1899300 .115307$

$81.202465-2.0467670 .659749$

$70.8807200 .063207-0.111709$

62.2127440 .5556930 .253033

12.4566130 .1091441 .222161

$10.2381770 .699855-0.570152$

62.1543772 .0698140 .391135

11.4301222 .3706381 .153347

13.1373572 .4531230 .676114

$11.8721102 .530108-0.562388$

$63.2524030 .118282-0.774685$

$13.255001-0.968928-0.891626$

$13.0388620 .575634-1.746622$

$14.2496580 .434519-0.454899$

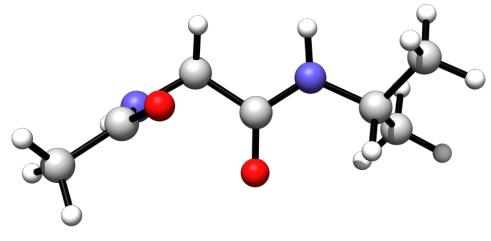

3B'

01

$\#$ imaginary freq. $=0$

$E=-534.992588$

$7-2.1437610 .6651980 .667321$
$1-2.6391291 .5464610 .683194$

$6-0.8105940 .5923201 .213847$

$1-0.6137091 .5166801 .761590$

$1-0.729863-0.2422071 .916396$

$6-2.646951-0.358663-0.045917$

$6-4.003331-0.168489-0.663992$

$1-4.660020-0.969156-0.315534$

$1-4.4453300 .798933-0.421678$

$1-3.903036-0.261451-1.748477$

$8-2.012600-1.419564-0.177978$

60.2521700 .4626840 .124947

$80.0777310 .928192-1.011098$

$71.381905-0.1479150 .500123$

$62.559548-0.256051-0.366413$

$12.191714-0.506259-1.366506$

$11.449833-0.5009471 .448403$

$63.440492-1.3853230 .146639$

$12.901291-2.3366600 .154935$

$14.319072-1.489965-0.494788$

$13.784953-1.1684931 .164125$

$63.3142421 .068745-0.427802$

$12.6592801 .878915-0.760051$

13.7131901 .3223330 .560150

$14.1502420 .990473-1.129106$

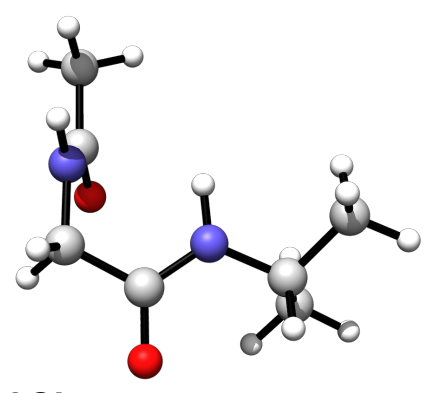

$3 C^{\prime}$

01

$\#$ imaginary freq. $=0$

$\mathrm{E}=-534.992464$

$7-1.8472940 .422313-0.755343$

$1-2.1643030 .240339-1.698625$

$6-1.0185721 .576702-0.492740$

$1-1.0366672 .226527-1.370044$

$1-1.4124052 .1414150 .356039$

$6-2.234397-0.4022600 .240327$

$6-3.119645-1.558396-0.130756$

$1-4.038776-1.4930540 .456509$

$1-3.364840-1.577711-1.193415$

$1-2.609108-2.4852680 .142770$

$8-1.863726-0.2116601 .409416$

$60.4418901 .279463-0.180394$ 
81.1500502 .1919490 .279721

$70.9072770 .059817-0.459293$

$62.273975-0.363383-0.139039$

$12.9306580 .472730-0.397724$

$10.252283-0.645687-0.778496$

$62.625554-1.570866-0.995291$

$12.559813-1.334623-2.060969$

$13.645103-1.896580-0.774352$

$11.946892-2.403413-0.777889$

$62.406917-0.6655801 .351067$

12.1162530 .1993101 .953856

$11.767367-1.5125191 .622139$

$13.442929-0.9227651 .590520$

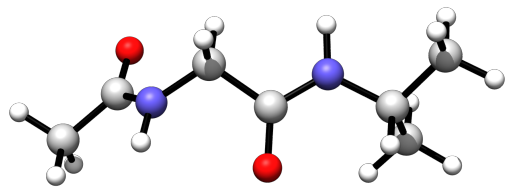

$3 A B$

01

\# imaginary freq. $=1$

$E=-534.989423$

$72.006972-0.0888610 .678210$

12.0981580 .6356491 .379419

$60.772906-0.8352580 .644032$

$10.805220-1.530565-0.195674$

$10.656979-1.4205701 .562653$

$62.955632-0.179733-0.273214$

$64.1379620 .740328-0.133085$

$14.1865401 .372952-1.023065$

14.0862281 .3697900 .756380

$15.0465790 .134465-0.100264$

$82.880206-0.985409-1.216392$

$6-0.4371940 .0836690 .533160$

$8-0.3667641 .2843840 .837110$

$7-1.572490-0.5098730 .147913$

$6-2.8523030 .1995030 .051493$

$1-2.9065410 .8664810 .917787$

$1-1.539996-1.485190-0.129053$

$6-3.982262-0.8169300 .122152$

$1-3.947659-1.3852231 .055806$

$1-4.945239-0.3034430 .064054$

$1-3.918604-1.517913-0.717692$

$6-2.9192391 .032585-1.225229$

$1-2.0805411 .731663-1.282373$

1 -2.892629 $0.379776-2.104189$

$1-3.8504141 .606478-1.248240$

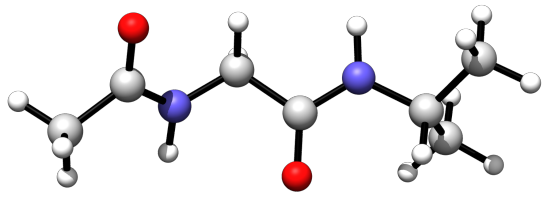

3AB'

01

\# imaginary freq. $=1$

$E=-534.989427$

$71.995893-0.4610270 .428550$

$12.042770-1.4671400 .327156$

60.7633360 .1098010 .914801

$10.580487-0.2032231 .948951$

10.8513291 .1965830 .903605

$63.0272070 .277479-0.022680$

$64.213028-0.480817-0.553623$

$15.098427-0.1785050 .010754$

$14.090068-1.562576-0.487061$

$14.364397-0.194890-1.597570$

83.0193301 .5198370 .001243

$6-0.432734-0.3413290 .087739$

$8-0.386648-1.364295-0.612476$

$7-1.5270960 .4186880 .207704$

$6-2.8163850 .069182-0.397501$

$1-2.601171-0.294433-1.407141$

$1-1.4851121 .2329960 .811321$

$6-3.6712401 .324858-0.482677$

$1-3.1820502 .098224-1.081349$

$1-4.6335061 .086803-0.942753$

$1-3.8621341 .7252260 .519381$

$6-3.508143-1.0356370 .395774$

$1-2.866947-1.9172640 .481880$

$1-3.754464-0.6807751 .402178$

$1-4.435539-1.330206-0.104261$

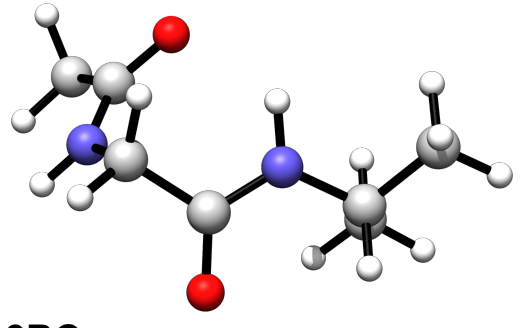

3BC

01

\# imaginary freq. $=1$

$E=-534.989368$

$7-2.0284540 .758916-0.030515$

$1-2.5727671 .2923720 .634820$

$6-0.9666671 .424131-0.765368$

$1-0.9316101 .027614-1.781918$

$1-1.1865822 .490721-0.801350$

$6-2.251644-0.563973-0.149568$

$6-3.367758-1.1421360 .674928$

$1-2.949481-1.9050161 .336341$

$1-3.886255-0.3890581 .269747$

$1-4.077675-1.6298050 .002511$ 
$8-1.571755-1.271638-0.911818$ $60.3700631 .238417-0.056980$ 80.6843091 .9656920 .900707 $71.1474380 .253482-0.520076$ $62.383597-0.1668640 .145495$ 12.8999670 .7476740 .451726 $10.744843-0.368703-1.214230$ $63.244398-0.929167-0.851149$ $13.493773-0.308649-1.716245$ $14.173921-1.246040-0.371416$ $12.718916-1.824702-1.201616$ $62.066441-1.0055061 .381041$ $11.419393-0.4563342 .071016$ $11.559848-1.9313001 .087214$ $12.989798-1.2674051 .906032$

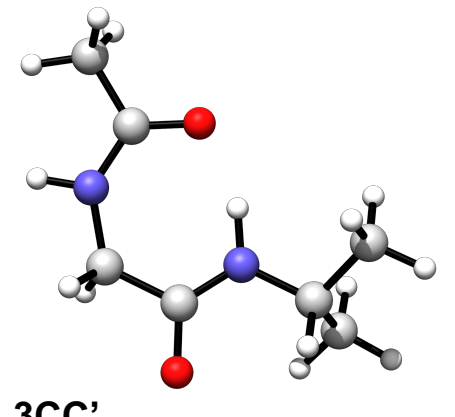

$3 C^{\prime}$

01

\# imaginary freq. $=1$

$E=-534.975771$

$7-2.0995010 .7989390 .013933$ $1-2.9499931 .3489120 .047372$ $6-0.9329251 .684582-0.103064$ $1-1.0823242 .293094-0.999245$ $1-0.9666962 .3700800 .748158$ $6-2.308803-0.5236890 .080438$ $6-3.750132-0.9487880 .191394$ $1-4.448809-0.1117510 .205164$ $1-3.978783-1.600661-0.655258$ $1-3.863190-1.5335751 .107468$ $8-1.417224-1.3960890 .057646$ $60.5257961 .252352-0.181301$ $81.3382782 .195501-0.276222$ $70.890656-0.021610-0.158955$ $62.294335-0.438118-0.220629$ $12.7880560 .198280-0.962066$ $10.148038-0.725870-0.070245$ $62.344926-1.888464-0.680089$ $11.880238-2.009888-1.662725$ $13.383815-2.221912-0.744339$ $11.822745-2.5340010 .035142$ $62.977894-0.2512281 .131281$ 12.9008370 .7849311 .471690
$12.515348-0.9022141 .880793$ $14.037651-0.5119081 .053671$

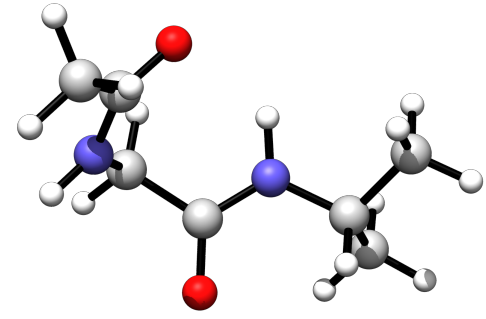

3B' C'

01

\# imaginary freq. $=1$

$E=-534.989038$

$72.048335-0.8412490 .060995$

$12.536015-1.492069-0.540712$

$60.896514-1.3068190 .812987$

$10.999829-2.3790800 .977570$

$10.869787-0.7952771 .776844$

62.4388020 .4477280 .084351

$63.6341360 .810205-0.752075$

$14.3766771 .287180-0.108153$

$14.079562-0.053491-1.247181$

$13.3202591 .538728-1.504065$

81.8425961 .2967170 .768106

$6-0.391363-1.0718560 .031850$

$8-0.755131-1.876058-0.843989$

$\begin{array}{llll}7 & -1.063161 & 0.045237 & 0.325007\end{array}$

$6-2.2906040 .448506-0.367890$

$1-2.1520550 .200342-1.425326$

$1-0.6627710 .6731331 .013799$

$6-2.4648281 .952611-0.217128$

$1-1.6111762 .494163-0.634576$

$1-3.3691662 .274437-0.739531$

$1-2.5672492 .2210460 .840292$

$6-3.493490-0.3201560 .171176$

$1-3.336175-1.3994550 .093487$

$1-3.664858-0.0652011 .222328$

$1-4.389616-0.058974-0.399349$

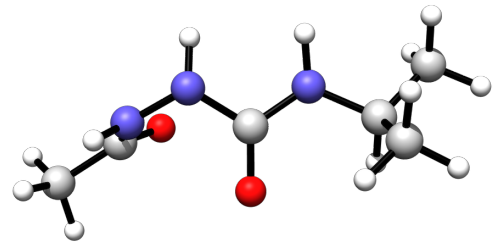

4A

01

$\#$ imaginary freq. $=0$

$E=-551.009849$

$7-2.031312-0.5507820 .757818$

$1-2.425717-1.4632950 .948519$ 
$6-2.6661550 .322033-0.064923$

$6-4.040266-0.085925-0.508315$

$1-4.035648-0.171484-1.597862$

$1-4.357068-1.033312-0.070627$

$1-4.7434970 .702262-0.230587$

$8-2.1376171 .383567-0.407712$ $60.234618-0.3304350 .133188$

$80.028118-0.883304-0.953033$

71.3854100 .2888660 .456881

$62.5856600 .168767-0.377554$ $12.2630820 .313150-1.412729$

$7-0.727043-0.3379101 .149863$ $1-0.6336680 .3899651 .853795$ 11.5081230 .6244791 .406377 63.5541561 .2772140 .007883 $13.1046242 .263927-0.133152$ 13.8528421 .1747641 .057400 $14.4540141 .213845-0.608987$ $63.222168-1.210882-0.236288$ $12.505069-2.000918-0.476485$ $14.075769-1.301750-0.914665$ $13.577600-1.3595890 .789086$

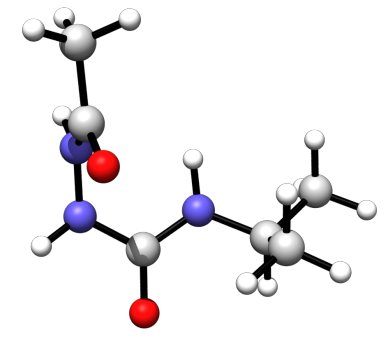

4B

01

$\#$ imaginary freq. $=0$

$E=-551.010498$

$71.7696180 .438223-0.784229$ $12.1574360 .359380-1.716506$ $62.127680-0.4220430 .207721$ $63.060562-1.527223-0.189923$ $12.571616-2.4812950 .021205$ $13.338699-1.483659-1.243613$ $13.957833-1.4588540 .429628$ $81.684363-0.2934161 .351257$ $6-0.3733001 .288114-0.162835$ $8-0.9829722 .1653640 .465742$ $7-0.9347870 .147832-0.602967$ $6-2.223223-0.309808-0.068713$ $1-2.8889620 .557353-0.065832$ $70.9552711 .524082-0.525944$ 11.3844152 .2248740 .074044 $1-0.323255-0.564530-0.987432$
$6-2.784860-1.376321-0.996726$ $1-2.932422-0.985781-2.007234$ $1-2.104615-2.234140-1.048054$ $1-3.746892-1.728593-0.616034$ $6-2.057700-0.8295071 .356922$ $1-1.621897-0.0632972 .004966$ $1-3.028277-1.1210531 .769185$ $1-1.400467-1.7062161 .362993$

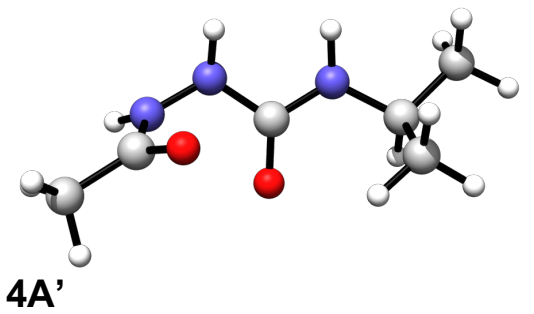

01

$\#$ imaginary freq. $=0$

$E=-551.009899$

$7-2.055400-0.972423-0.064000$ $1-2.579928-1.6430250 .484006$ $6-2.5246240 .285424-0.263678$ $6-3.9194460 .5464460 .223449$ $1-4.4964900 .986352-0.592446$ $1-4.415375-0.3566650 .581336$ 1 -3.864664 1.2759561 .035919 $8-1.8351201 .147310-0.816964$ $60.243406-0.6030180 .318633$ $80.020048-0.0967801 .424135$ $71.451850-0.614311-0.274427$ 62.5839240 .1361860 .277993 $12.581587-0.0430081 .356992$ $7-0.753029-1.299587-0.377459$ $1-0.600157-1.401183-1.377772$ $11.520652-0.923588-1.238152$ $63.870182-0.411353-0.322797$ $13.994487-1.473489-0.094842$ $13.867213-0.284085-1.411337$ 14.7277710 .1334320 .079942 62.4294471 .6316770 .014717 11.4797592 .0026960 .410793 13.2440272 .1848290 .491745 $12.4604081 .829594-1.062266$ 


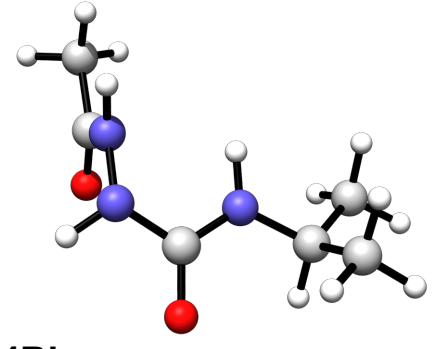

4B'

01

\# imaginary freq. $=0$

$E=-551.010077$

$71.695438-0.411953-0.830362$

$11.906664-0.295020-1.814192$

62.3752920 .2823880 .122244

$63.3346531 .317368-0.386218$

14.3292021 .0836450 .000921

$13.3666571 .361524-1.475533$

13.0334622 .2895370 .011321

82.1903750 .0678421 .322336

$6-0.402484-1.1244130 .071612$

$8-1.017939-2.0096460 .686635$

$7-0.8716210 .113105-0.136885$

$6-2.2226170 .5097750 .271723$

$1-2.3929300 .0763481 .261860$

$70.837393-1.439006-0.490193$

$11.287761-2.213969-0.008730$ $1-0.3190480 .763588-0.683554$

$6-2.2736712 .0273750 .369060$

$1-1.5476642 .4030281 .095393$

$1-2.0611092 .479955-0.606074$

$1-3.2722242 .3441860 .680254$

$6-3.268317-0.030628-0.699296$

$1-3.192463-1.117750-0.791194$

$1-4.2732920 .217314-0.345039$

$1-3.1301040 .415833-1.689883$

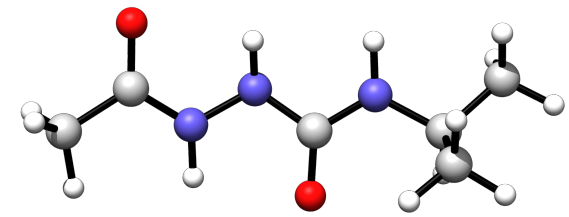

4AA'

01

\# imaginary freq. $=1$

$E=-551.002671$

$7-1.894151-0.302771-0.253895$

$1-1.796836-1.263136-0.564738$

$6-3.0482410 .2500220 .135611$

$6-4.246156-0.6530800 .147028$

$1-4.993674-0.242440-0.536295$

$1-4.005460-1.675318-0.148642$
$1-4.668912-0.6542071 .154366$ $8-3.1019431 .4481060 .471779$ $60.445498-0.232490-0.357531$ $80.451272-1.443284-0.629741$ $71.5509520 .499777-0.138494$ $62.888836-0.100325-0.126977$ $12.928631-0.797468-0.969500$ $7-0.7467160 .479166-0.348126$ $1-0.8102801 .3040020 .245241$ 11.4543951 .4655170 .154886 $63.9144671 .003948-0.337041$ $13.7531881 .520581-1.287131$ 13.8569551 .7371330 .475458 $14.9208240 .577977-0.339231$ $63.140760-0.8650061 .169307$ $12.374608-1.6287851 .328841$ $14.117062-1.3573311 .130357$ $13.133282-0.1767162 .021303$

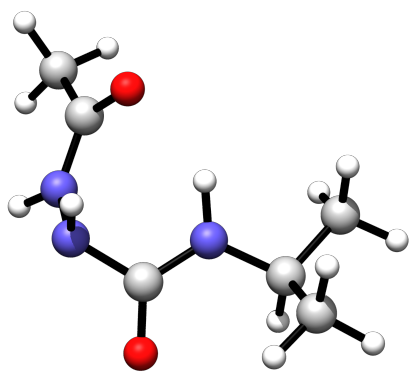

$4 A B$

01

\# imaginary freq. $=1$

$\mathrm{E}=-550.993372$

$7-1.881796-0.809917-0.246668$

$1-2.437003-1.352417-0.897723$

$6-2.3116690 .4029750 .166364$

$6-3.5136880 .955690-0.540012$ $1-3.2610751 .945259-0.927049$ $1-3.8526560 .316538-1.356178$ $1-4.3177201 .0698100 .191562$ $8-1.7367151 .0119161 .080871$ $60.451264-1.214974-0.064098$ $81.114206-2.107980-0.593344$ 70.8685080 .0421740 .094068 $62.1850030 .499388-0.364618$ $12.3608490 .026231-1.336024$ $7-0.882894-1.5227570 .432988$ $1-0.941565-1.2840081 .425647$ 10.2474070 .6957790 .565502

$62.1426552 .010872-0.533973$

$11.3786062 .307724-1.257890$ 11.9258662 .4964210 .424011 $13.1127682 .370072-0.886306$ 
63.2767610 .0663210 .609127

$13.266189-1.0174610 .754393$

14.2581520 .3538780 .220844

13.1315740 .5525571 .579539

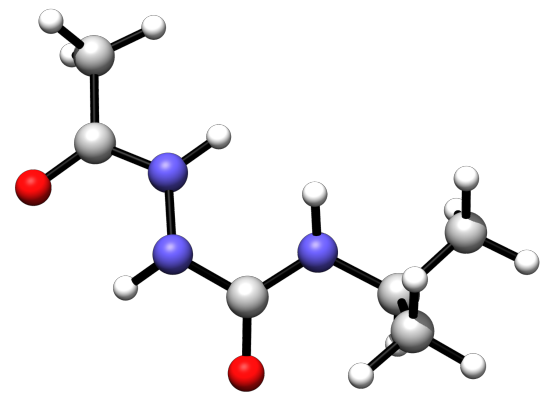

4BB'

01

\# imaginary freq. $=1$

$E=-550.996676$

7 -1.589352 0.2941050 .110051

$1-1.1232251 .1910520 .163371$

$6-2.9273240 .1929070 .069082$

$6-3.6895151 .4845480 .051139$

$1-4.3361841 .5136180 .931646$

$1-3.0380612 .3597840 .049207$

$1-4.3235351 .498628-0.838312$

$8-3.480239-0.9197020 .039208$

$60.510496-0.881561-0.190085$

$81.002625-1.936471-0.617595$

$71.2099180 .262614-0.032968$

$62.6658150 .282070-0.235116$

$12.860311-0.308648-1.133827$

$7-0.825639-0.8745070 .181597$

$1-1.348162-1.659644-0.200967$

10.8546650 .9743780 .597208

$63.1018861 .720927-0.469283$

$12.6068192 .147922-1.345573$

12.8640822 .3390340 .403985

$14.1827191 .758642-0.627326$

$63.392631-0.3421660 .952208$

$13.047398-1.3641911 .131221$

$14.469672-0.3684670 .762177$

13.2159970 .2514421 .855863

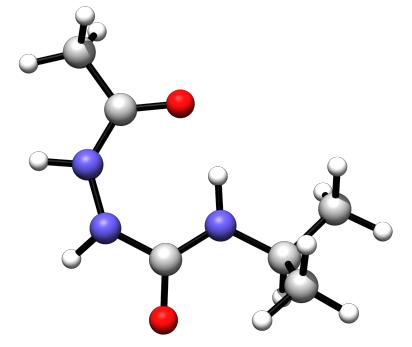

4BB'-2
01

\# imaginary freq. $=1$

$E=-550.985321$

72.0531690 .8059530 .037306

12.8432501 .4265250 .168819

$62.313603-0.5094220 .064475$

$63.778935-0.8437710 .149882$

14.4283510 .0317600 .125081

$13.945780-1.3933821 .079742$

$14.023855-1.503914-0.685045$

$81.462044-1.4161430 .021307$

$6-0.4673101 .208712-0.171222$

$8-1.2311992 .160390-0.433136$

$7-0.876186-0.052791-0.032524$

$6-2.278739-0.429163-0.230455$

$1-2.6593560 .151327-1.077231$

70.8625101 .5561630 .063047

$11.0492352 .475305-0.323307$

$1-0.163351-0.7781000 .077337$

$6-2.328956-1.911607-0.573121$

$1-1.758782-2.128230-1.481150$

$1-1.916929-2.5080750 .248636$

$1-3.365037-2.220917-0.732318$

$6-3.113477-0.1096291 .006101$

$1-3.0298140 .9471881 .274382$

$1-4.167137-0.3351270 .815621$

$1-2.777610-0.7143351 .855304$

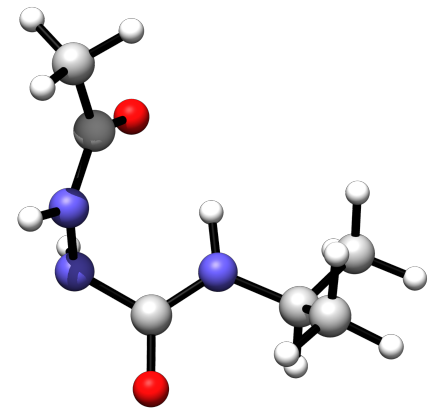

4A'B'

01

\# imaginary freq. $=1$

$E=-550.993459$

71.8143870 .7644700 .257774

12.2998991 .3084410 .960820

$62.228371-0.486962-0.038937$

$63.289794-1.0603040 .852188$

$14.183630-1.2385620 .248813$

$13.539419-0.4029361 .686035$

$12.937804-2.0215461 .232767$

$81.750362-1.112760-0.996961$

$6-0.4474731 .280766-0.258505$

$8-1.1333642 .2411940 .093437$ 
$7-0.9055770 .034689-0.392237$ $6-2.274806-0.345499-0.026272$ $1-2.9323760 .446993-0.397230$ $70.9574541 .494954-0.577735$ $11.1369281 .216907-1.545125$ $1-0.252194-0.685660-0.690990$ $6-2.615611-1.655709-0.720453$ $1-2.539625-1.560193-1.807118$ $1-1.937984-2.450599-0.389468$ $1-3.636996-1.951691-0.468707$ $6-2.418145-0.4499821 .489082$ $1-2.1256120 .4841451 .976886$ $1-3.457781-0.6662001 .751696$ $1-1.787728-1.2592341 .872576$

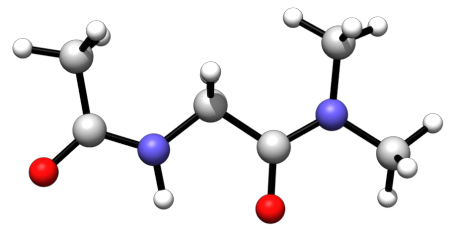

1A-E

01

$\#$ imaginary freq. $=0$

$\mathrm{E}=-495.675234$

$7-1.356861-0.499709-0.001390$ $1-1.156906-1.4956640 .019485$ $6-0.2156770 .389262-0.029068$ $1-0.2348931 .025991-0.920020$ $1-0.2040231 .0434260 .850387$ $6-2.643155-0.1108730 .008196$ $6-2.9122761 .368241-0.015065$ $1-2.4517061 .8512020 .852072$ $1-2.4807541 .817761-0.914555$ $1-3.9878821 .537557-0.000806$ $8-3.563918-0.9490740 .034231$ $61.055468-0.448344-0.036053$ $80.986563-1.6883930 .016973$ $72.2260250 .203482-0.111905$ $63.454528-0.5740740 .019584$ $14.2859340 .029029-0.345488$ $13.638426-0.8421891 .065719$ $13.385621-1.485634-0.573326$ 62.3527721 .6475830 .061368 12.5548191 .8950931 .109024 $13.1841062 .000434-0.550853$ $11.4519822 .165548-0.261980$

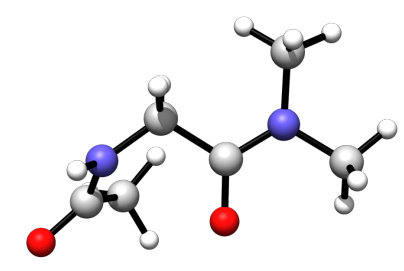

1B-E

01

$\#$ imaginary freq. $=0$

$\mathrm{E}=-495.675113$

$71.4824670 .882133-0.550016$

$11.7270471 .153540-1.496082$

$60.1174591 .108529-0.134050$ 10.0819801 .4877600 .890244 $1-0.2978331 .889130-0.775800$ 62.3583470 .0414190 .038804 $62.034507-0.5416551 .386921$ $12.887143-0.3595292 .044911$ $11.131577-0.1412331 .846168$ $11.923814-1.6227611 .268276$ $83.439066-0.227745-0.515215$ $6-0.747289-0.148514-0.252777$ $8-0.262796-1.211560-0.671052$ $7-2.032700-0.0479140 .131099$ $6-2.908662-1.189938-0.116033$ $1-3.152016-1.276264-1.180791$ $1-2.425831-2.1105520 .211243$ $1-3.829194-1.0486420 .450015$ $6-2.6880841 .2344880 .378467$ $1-3.5223411 .0694801 .061038$ $1-2.0071721 .9425810 .847500$ $1-3.0733791 .665422-0.552163$

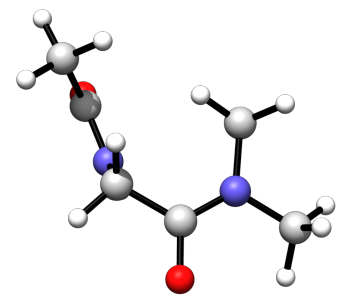

1C-E

01

$\#$ imaginary freq. $=0$

$\mathrm{E}=-495.671407$

$7-1.045559-0.492965-0.582121$

$1-0.756771-0.430430-1.553594$

$6-0.144000-1.1520290 .345806$

$1-0.404603-0.8865891 .372520$

$1-0.205474-2.2383420 .250431$

$6-2.2416360 .059036-0.296567$ 
$6-2.752342-0.0402821 .115972$ $1-3.7600480 .3712321 .148735$ $1-2.767614-1.0807951 .451022$ $1-2.1109110 .5255241 .799060$ 8 -2.900693 $0.621897-1.188607$ $61.311389-0.7796920 .077404$ $82.132990-1.686269-0.156404$ 71.6596640 .5146980 .084445 $63.0249310 .882110-0.277052$ 13.6866940 .8420180 .594439 $13.4028580 .205694-1.043052$ $13.0155571 .899810-0.669825$ 60.7973601 .6080120 .525430 $10.3818042 .151879-0.328206$ $1-0.0128041 .2438771 .153735$ 11.3999802 .2960321 .122588

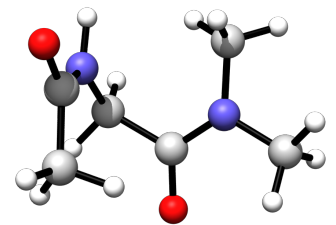

1D-E

01

\# imaginary freq. $=0$

$E=-495.673088$

71.2763610 .4042980 .881913 11.3657941 .3487221 .240700 $60.212935-0.4300581 .413978$ $10.591343-1.4180111 .681312$ $1-0.1513140 .0409392 .329886$ $61.9987560 .137643-0.227378$ $61.802014-1.197999-0.891427$ $12.643168-1.381866-1.558900$ $11.717878-2.010520-0.166163$ $10.881893-1.177256-1.485963$ $82.7949400 .978312-0.678169$ $6-0.960569-0.6605160 .461780$ $8-1.341860-1.8267290 .246506$ $7-1.5403540 .399831-0.117412$ $6-2.5757430 .180167-1.121968$ $1-2.5837851 .032898-1.802402$ $1-2.358242-0.726579-1.686167$ $1-3.5625290 .084291-0.657227$ $6-1.2601161 .7894210 .235716$ $1-0.8827911 .8675771 .254647$ $1-0.5439342 .239883-0.458433$ $1-2.1978342 .3464200 .186300$

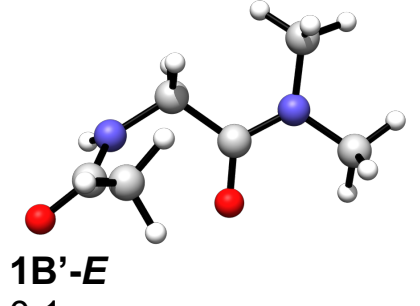

01

\# imaginary freq. $=0$

$E=-495.675106$

$7-1.5088700 .838655-0.610552$

$1-1.8053411 .032401-1.560866$

$6-0.1279851 .112147-0.285143$

$10.2354871 .861857-0.991014$ $1-0.0461001 .5487760 .713668$ $6-2.3358980 .0279480 .082497$

$6-1.929692-0.4529551 .448854$ $1-1.745065-1.5288441 .385610$ $1-1.0427760 .0333051 .853883$ $1-2.772088-0.2937622 .125213$ $8-3.437359-0.299730-0.393851$ $60.752189-0.137324-0.372411$ $80.263611-1.232779-0.691253$ $72.0595160 .012198-0.095408$ $62.884491-1.190179-0.019187$ $13.932872-0.894985-0.055280$ $12.697674-1.7373400 .911496$ $12.669064-1.845638-0.862704$ 62.6094361 .2263070 .503492 12.5118161 .2079801 .594549 13.6673061 .2891550 .246440 12.1173712 .1155350 .112949

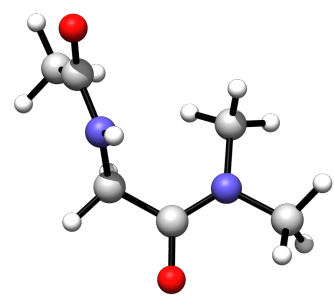

$1 C^{\prime}-E$

01

\# imaginary freq. $=0$

$E=-495.671407$

$71.045536-0.492837-0.582160$ $10.756859-0.430554-1.553680$ $60.144040-1.1520460 .345707$ $10.205491-2.2383480 .250201$ $10.404673-0.8867431 .372443$

$62.2416670 .059069-0.296572$ 
$62.752307-0.0403501 .115992$ 12.1108530 .5254311 .799082

$12.767485-1.0808951 .450952$ 13.7600150 .3711391 .148871 $82.9007600 .621930-1.188565$ $6-1.311356-0.7796820 .077355$ $8-2.133009-1.686261-0.156254$ 7 -1.659599 0.5147250 .084220 $6-3.0249240 .882054-0.277120$ 1 -3.402703 $0.205950-1.043472$ $1-3.6867130 .8414060 .594329$ 1 -3.015714 $1.899947-0.669389$ $6-0.7974581 .6080140 .525607$ 10.0128381 .2437411 .153651 $1-0.3821592 .152373-0.327837$ $1-1.4001412 .2956371 .123169$

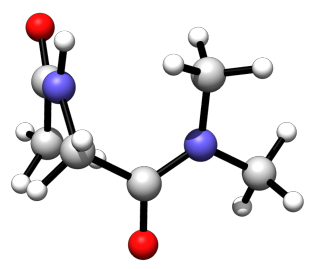

1D'-E

01

\# imaginary freq. $=0$

$E=-495.673088$

$7-1.2763680 .4042680 .881932$ $1-1.3658241 .3486811 .240743$

$6-0.212934-0.4300831 .413985$ 10.1513120 .0409072 .329898 $1-0.591330-1.4180441 .681308$ $6-1.9987400 .137633-0.227380$

$6-1.801971-1.197988-0.891462$ $1-0.881853-1.177212-1.486001$ $1-1.717820-2.010528-0.166223$ $1-2.643122-1.381852-1.558940$ $8-2.7949280 .978303-0.678162$ $60.960571-0.6605160 .461782$ $81.341878-1.8267220 .246498$ $71.5403380 .399843-0.117404$ $62.5757190 .180197-1.121973$ $12.5837571 .032943-1.802389$ $13.5625100 .084308-0.657244$ $12.358210-0.726536-1.686190$ 61.2600791 .7894270 .235730 $10.5438882 .239880-0.458417$ 10.8827541 .8675731 .254661 12.1977882 .3464420 .186314

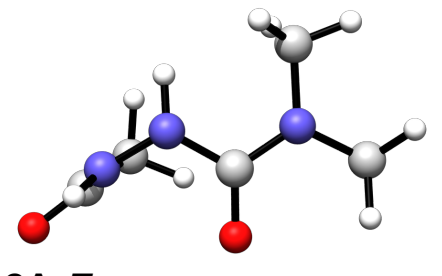

2A-E

01

$\#$ imaginary freq. $=0$

$E=-511.693860$

$71.4731280 .348456-0.924334$

$11.7913140 .077823-1.849329$

62.2765510 .0645170 .137906

61.7239780 .3272301 .509687

12.5227440 .1885422 .236671

11.3274691 .3436111 .580107

$10.909658-0.3704531 .728343$

$83.419504-0.358261-0.060117$

$6-0.732515-0.354044-0.404585$

$8-0.353107-1.534188-0.378848$

$7-2.0013550 .033217-0.129973$

$6-2.878594-0.9491940 .501500$

$1-2.702341-1.0027371 .582152$

$1-3.912994-0.6541620 .323848$

$1-2.711493-1.9313430 .063351$

$6-2.3337451 .4422660 .074151$

$1-2.0984412 .033929-0.813708$

$1-3.4075241 .5147270 .243182$

$1-1.8146431 .8621880 .944082$

$70.1402680 .657027-0.787041$

$1-0.0532661 .621906-0.549495$

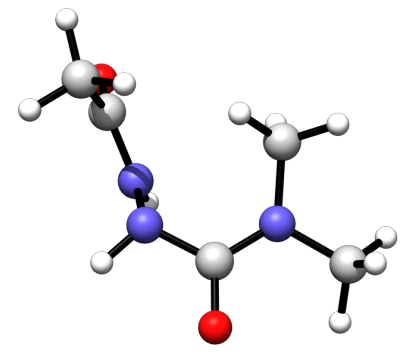

2B-E

01

$\#$ imaginary freq. $=0$

$E=-511.688987$

$7-1.009952-0.370447-0.720014$

$1-0.814549-0.185673-1.701195$

$6-2.1778250 .081412-0.201490$

$6-2.513166-0.2972501 .211802$

$1-2.719911-1.3711361 .255147$ 
$1-1.686696-0.0938451 .894974$ $1-3.4003620 .2563041 .515887$

$8-2.9348020 .752362-0.914170$ $61.243284-0.766733-0.007595$ $82.091792-1.674698-0.007119$ $71.5582470 .542392-0.009249$ $62.9716220 .892371-0.115608$ $13.0433031 .908700-0.505048$ 13.4665540 .8473120 .860788 $13.4731120 .212925-0.803442$ 60.6671061 .5960270 .482352 $10.1530312 .112254-0.332283$ $1-0.0655871 .1898041 .179080$ 11.2782042 .3171431 .028370 $7-0.109347-1.1318420 .004365$ $1-0.201768-2.130778-0.164444$

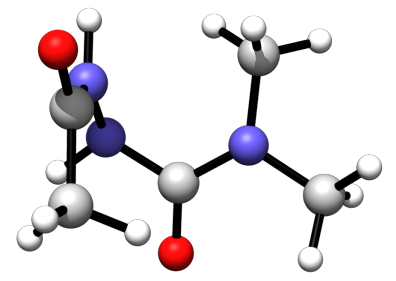

$2 C-E$

01

$\#$ imaginary freq. $=0$

$E=-511.690804$

$7-1.2007180 .302285-0.892234$

$1-1.3336341 .146624-1.438891$

$6-1.9827710 .1119280 .203193$

$6-1.752278-1.1340011 .010631$

$1-2.555297-1.2241741 .740691$

$1-1.727705-2.0198660 .370448$

$1-0.795383-1.0726401 .539422$

$8-2.8451480 .9503950 .486092$

$60.962694-0.664187-0.436642$

$81.479605-1.787561-0.311456$

71.4471210 .4478080 .142272

62.5431050 .3045201 .094363

12.4997861 .1385351 .796608

$12.436919-0.6295851 .644924$

13.5135040 .3152650 .586241

$61.1480781 .803901-0.315724$

$10.8551411 .798864-1.366177$

10.3624602 .2734300 .283291

$12.0621132 .394257-0.225602$

$7-0.165151-0.537662-1.258881$

$1-0.475292-1.443365-1.601075$

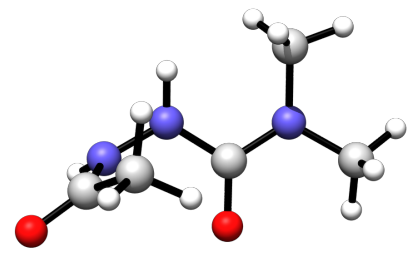

$2 A^{\prime}-E$

01

\# imaginary freq. $=0$

$E=-511.693860$

$7-1.4730950 .348818-0.924147$

$1-1.7913260 .078872-1.849323$

$6-2.2766140 .0644480 .137911$

$6-1.7241080 .3264081 .509854$

$1-0.909518-0.3711041 .728055$

$1-1.3279351 .3428761 .580921$

$1-2.5228110 .1870032 .236766$

$8-3.419625-0.358034-0.060408$

$60.732541-0.353910-0.404675$

$80.353123-1.534046-0.379394$

$72.0014120 .033255-0.129986$

$62.878644-0.9493290 .501227$

$13.913049-0.6543850 .323441$

$12.702558-1.0029731 .581901$

$12.711373-1.9314030 .062979$

62.3338451 .4422370 .074540

11.8147421 .8619360 .944580

13.4076211 .5146060 .243628

$12.0985552 .034159-0.813151$

$7-0.1402340 .657331-0.786703$

$10.0532721 .622096-0.548665$

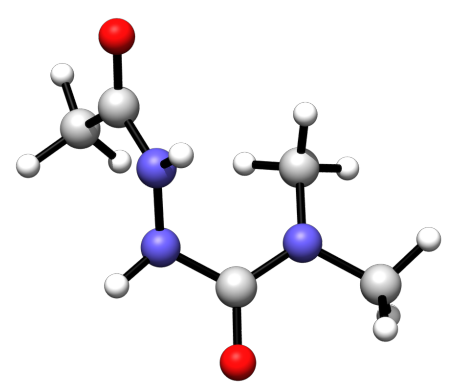

2B'-E

01

$\#$ imaginary freq. $=0$

$E=-511.688987$

$71.009997-0.370220-0.720060$

$10.814761-0.185292-1.701247$

$62.1779010 .081396-0.201436$ 
$62.513187-0.2973861 .211844$ 13.4004610 .2560481 .515922 $11.686804-0.0936531 .895052$ $12.719705-1.3712921 .255282$ $82.9349960 .752386-0.913968$ $6-1.243249-0.766709-0.007714$ $8-2.091689-1.674769-0.007045$ $7-1.5583510 .542388-0.009480$ $6-2.9717940 .892226-0.115413$ $1-3.0436861 .908574-0.504766$ $1-3.4734050 .212797-0.803169$ $1-3.4664400 .8470400 .861122$ $6-0.6672701 .5960070 .482297$ 10.0652601 .1897271 .179157 $1-0.1529562 .112156-0.332237$ $1-1.2784472 .3171901 .028126$ $70.109353-1.1317100 .004122$ $10.201849-2.130642-0.164673$

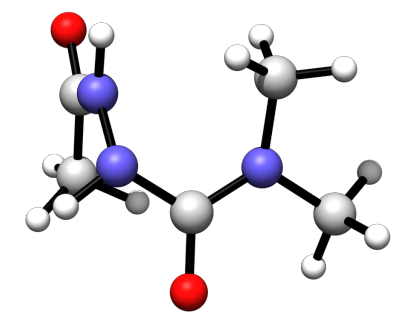

2C'-E

01

\# imaginary freq. $=0$

$E=-511.690804$

$71.2006640 .302488-0.892138$ $11.3335121 .146889-1.438720$ 61.9828490 .1120180 .203179 $61.752590-1.1340651 .010434$ $10.795883-1.0727531 .539578$ $11.727706-2.0198210 .370114$ $12.555865-1.2244291 .740191$ 82.8452020 .9505030 .486111 $6-0.962674-0.664206-0.436617$ $8-1.479473-1.787634-0.311509$ $7-1.4471940 .4477150 .142359$ $6-1.1483611 .803851-0.315641$ $1-2.0625272 .394034-0.225690$ $1-0.3629402 .2735540 .283493$ $1-0.8552641 .798857-1.366048$ $6-2.5432720 .3043441 .094332$ $1-2.437248-0.6298961 .644689$ $1-2.4999271 .1382001 .796767$ $1-3.5136210 .3153200 .586116$ $70.165180-0.537537-1.258849$
$10.475395-1.443218-1.601039$

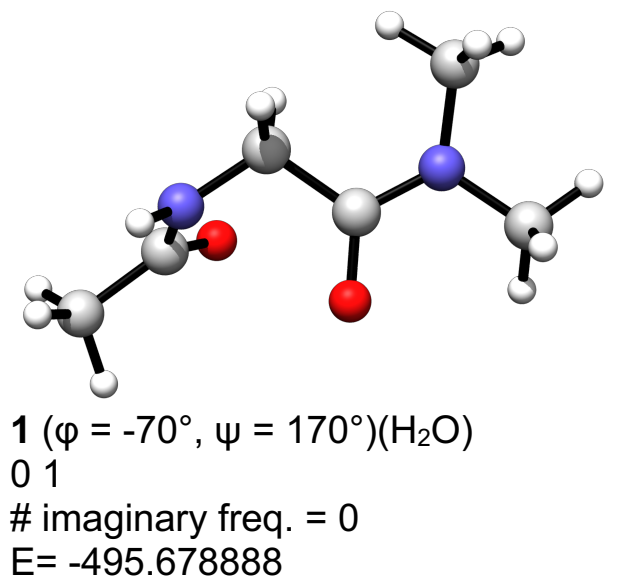

$71.4781220 .679222-0.668216$ $11.8131550 .703378-1.622083$ $60.1063161 .030278-0.398543$ 10.0579371 .5968880 .533962 $1-0.2557101 .673240-1.204121$ 62.2318280 .0713470 .266975 $63.611680-0.361624-0.142417$ $13.682794-1.445340-0.019116$ $13.847309-0.097515-1.174034$ 14.3369090 .1029900 .529900 $81.809623-0.1088861 .421575$ $6-0.792499-0.204732-0.300084$ $8-0.364238-1.326508-0.614619$ $7-2.047104-0.0239860 .152403$ $6-2.950779-1.1703740 .132705$ $1-3.271478-1.400992-0.889324$ $1-2.457809-2.0458300 .554766$ $1-3.826820-0.9283380 .734817$ $6-2.6908341 .2832090 .251395$ $1-3.3554271 .2844141 .117336$ $1-1.9617292 .0797310 .380206$ $1-3.2836041 .485255-0.647457$

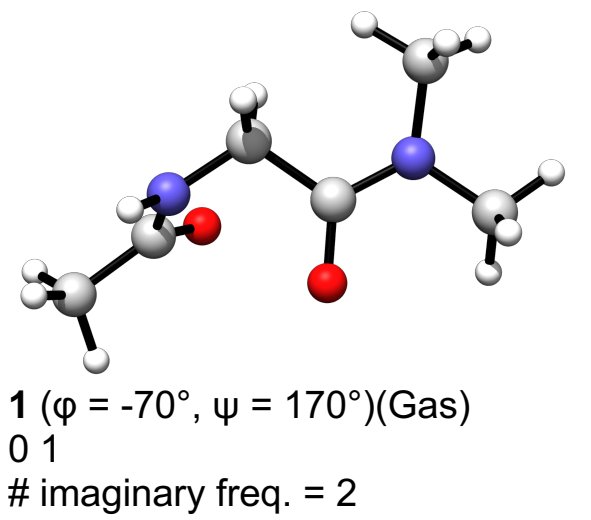


$E=-495.645750$

$71.4781220 .679222-0.668216$ $11.8131550 .703378-1.622083$ $60.1063161 .030278-0.398543$ 10.0579371 .5968880 .533962 $1-0.2557101 .673240-1.204121$ 62.2318280 .0713470 .266975 $63.611680-0.361624-0.142417$ $13.682794-1.445340-0.019116$ $13.847309-0.097515-1.174034$ 14.3369090 .1029900 .529900 $81.809623-0.1088861 .421575$ $6-0.792499-0.204732-0.300084$ $8-0.364238-1.326508-0.614619$ $7-2.047104-0.0239860 .152403$ $6-2.950779-1.1703740 .132705$ $1-3.271478-1.400992-0.889324$ $1-2.457809-2.0458300 .554766$ $1-3.826820-0.9283380 .734817$ $6-2.6908341 .2832090 .251395$ $1-3.3554271 .2844141 .117336$ $1-1.9617292 .0797310 .380206$ $1-3.2836041 .485255-0.647457$

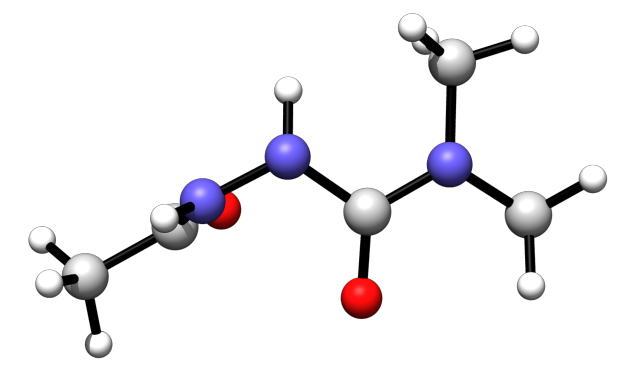

$2\left(\varphi=-70^{\circ}, \Psi=170^{\circ}\right)\left(\mathrm{H}_{2} \mathrm{O}\right)$

01

\# imaginary freq. $=0$

$E=-511.696316$
71.415336
0.528014
11.818200
0.579573
62.135641
0.085836
63.570910
$-0.247384$
13.720921
$-1.310961$
13.853809
$-0.031652$
0.323414
14.204252
$81.630447-0.028651$
$-0.753321$
$-1.680281$
0.306448
0.021789
0.224492
$-1.009404$
0.704331
1.427378
$6-0.778973-0.269841$
$-0.438357$
$8-0.382677$
$-1.428552$
$7-2.061254$
0.039945
$6-2.897871$
$-1.048333$
$1-2.680630-1.259688$
$1-3.943286$
$-0.753980$
$-0.618995$
$-0.124673$
0.376294
1.429830
0.280013

$\begin{array}{cccc}1-2.729218 & -1.948106 & -0.212791 \\ 6 & -2.439266 & 1.391675 & 0.282171 \\ 1-2.122622 & 2.125887 & -0.461107 \\ 1-3.526450 & 1.434563 & 0.345410 \\ 1-2.021784 & 1.655539 & 1.261794 \\ 70.074929 & 0.820480 & -0.631021 \\ 1-0.101066 & 1.652241 & -0.076319\end{array}$

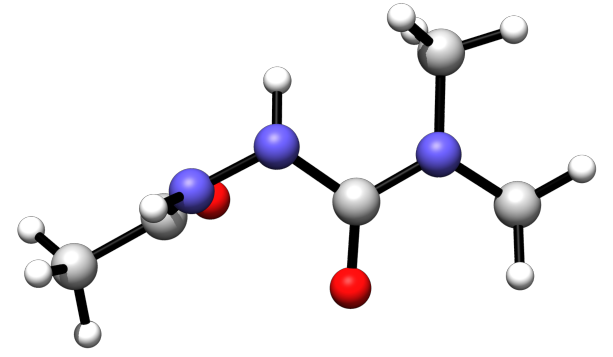

$2\left(\varphi=-70^{\circ}, \psi=170^{\circ}\right)($ Gas $)$

01

\# imaginary freq. $=0$

$E=-550.975299$

7 -2.001622 -0.478119 0.767287

$1-2.377866-1.3228161 .179746$

$6-2.7326800 .275228-0.090282$

$6-4.121036-0.219997-0.371965$

$1-4.216608-0.382463-1.448106$

$1-4.352937-1.1442370 .158973$

$1-4.8304570 .557708-0.079485$

$8-2.2723061 .303286-0.596568$

$60.258753-0.2358370 .123718$

$80.042832-0.832558-0.937791$

71.4407250 .3212560 .449132

$62.6405450 .125621-0.369982$

$12.3313460 .243797-1.412595$

$7-0.719593-0.1222641 .112884$

$1-0.6596270 .6847391 .728170$

11.5656660 .6837191 .388166

$63.6490081 .209211-0.017372$

$13.2403292 .206616-0.201058$

13.9335681 .1360351 .038493

$14.5514571 .086196-0.621374$

$63.221872-1.271464-0.172839$

$12.475865-2.041098-0.390150$

$14.076629-1.420839-0.839316$

$13.563085-1.3958700 .860624$ 


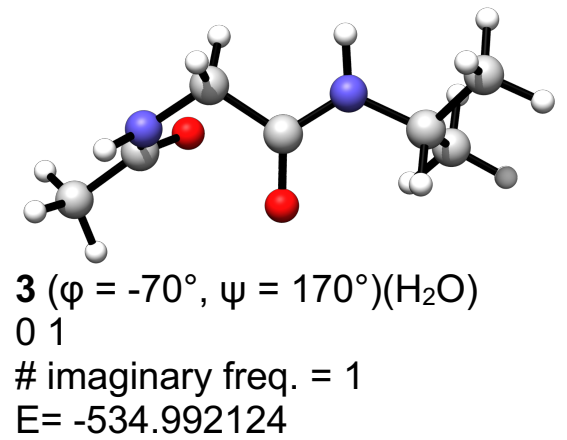

$72.111324-0.9657840 .026498$ $12.552756-1.5828840 .694629$ $60.783201-1.270202-0.439707$ $10.707518-1.083016-1.514329$ $10.589799-2.331346-0.268634$ $62.6815110 .223802-0.240936$ 64.0321530 .4876640 .363104 13.9630511 .3836590 .984965 $14.394175-0.3464450 .965753$ $14.7404790 .690315-0.443936$ $82.1180461 .060113-0.967146$ $6-0.294162-0.4633070 .285983$ $8-0.0509720 .1880761 .311514$ $7-1.517204-0.553983-0.251591$ $6-2.6823730 .1522350 .289171$ $1-2.6054830 .0926101 .379136$ $1-1.626838-1.063801-1.121957$ $6-3.946585-0.559517-0.169243$ 1 -3.962807 -1.598520 0.171336 $1-4.825188-0.0489950 .233166$ $1-4.016984-0.547643-1.262773$ $6-2.6714531 .618528-0.133966$ $1-1.7397002 .1057330 .166919$ $1-2.7731231 .698089-1.221593$ $1-3.5068412 .1498880 .331587$

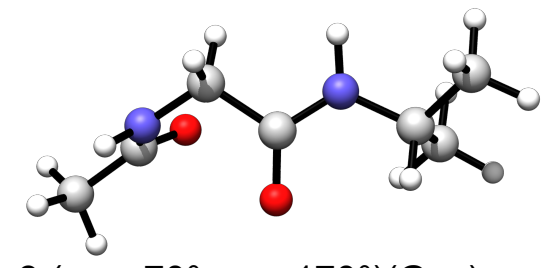

$3\left(\varphi=-70^{\circ}, \psi=170^{\circ}\right)($ Gas $)$

01

\# imaginary freq. $=1$

$E=-534.958703$

$72.111324-0.9657840 .026498$ $12.552756-1.5828840 .694629$ $60.783201-1.270202-0.439707$ $10.707518-1.083016-1.514329$
$10.589799-2.331346-0.268634$ $62.6815110 .223802-0.240936$ 64.0321530 .4876640 .363104 13.9630511 .3836590 .984965 $14.394175-0.3464450 .965753$ $14.7404790 .690315-0.443936$ $82.1180461 .060113-0.967146$ $6-0.294162-0.4633070 .285983$ $8-0.0509720 .1880761 .311514$ $7-1.517204-0.553983-0.251591$ $6-2.6823730 .1522350 .289171$ $1-2.6054830 .0926101 .379136$ $1-1.626838-1.063801-1.121957$ $6-3.946585-0.559517-0.169243$ $1-3.962807-1.5985200 .171336$ $1-4.825188-0.0489950 .233166$ $1-4.016984-0.547643-1.262773$ $6-2.6714531 .618528-0.133966$ $1-1.7397002 .1057330 .166919$ $1-2.7731231 .698089-1.221593$ $1-3.5068412 .1498880 .331587$

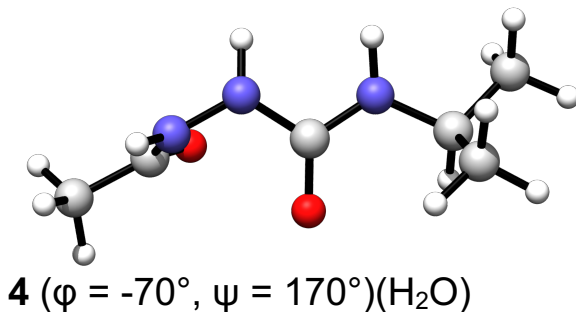

01

\# imaginary freq. $=0$

$E=-551.009236$

$7-2.001622-0.4781190 .767287$ $1-2.377866-1.3228161 .179746$ $6-2.7326800 .275228-0.090282$ $6-4.121036-0.219997-0.371965$ $1-4.216608-0.382463-1.448106$ $1-4.352937-1.1442370 .158973$ $1-4.8304570 .557708-0.079485$ $8-2.2723061 .303286-0.596568$ $60.258753-0.2358370 .123718$ $80.042832-0.832558-0.937791$ 71.4407250 .3212560 .449132 $62.6405450 .125621-0.369982$ $12.3313460 .243797-1.412595$ $7-0.719593-0.1222641 .112884$ $1-0.6596270 .6847391 .728170$ 11.5656660 .6837191 .388166 $63.6490081 .209211-0.017372$ $13.2403292 .206616-0.201058$ 
13.9335681 .1360351 .038493

$14.5514571 .086196-0.621374$

$63.221872-1.271464-0.172839$

$12.475865-2.041098-0.390150$

$14.076629-1.420839-0.839316$

$13.563085-1.3958700 .860624$

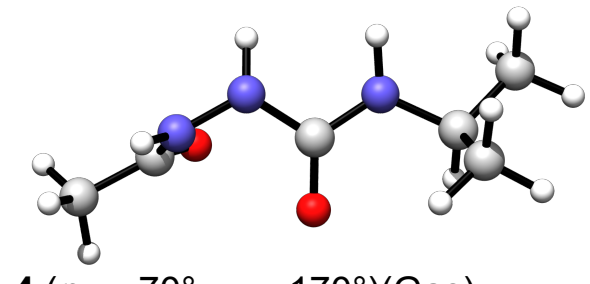

$4\left(\varphi=-70^{\circ}, \psi=170^{\circ}\right)($ Gas $)$

01

$\#$ imaginary freq. $=0$

$\mathrm{E}=-550.975299$

$7-2.001622-0.4781190 .767287$

$1-2.377866-1.3228161 .179746$

$6-2.7326800 .275228-0.090282$

$6-4.121036-0.219997-0.371965$

$1-4.216608-0.382463-1.448106$

$1-4.352937-1.1442370 .158973$

$1-4.8304570 .557708-0.079485$

$8-2.2723061 .303286-0.596568$

$60.258753-0.2358370 .123718$

$80.042832-0.832558-0.937791$

71.4407250 .3212560 .449132

$62.6405450 .125621-0.369982$

$12.3313460 .243797-1.412595$

$7-0.719593-0.1222641 .112884$

$1-0.6596270 .6847391 .728170$

11.5656660 .6837191 .388166

$63.6490081 .209211-0.017372$

$13.2403292 .206616-0.201058$

13.9335681 .1360351 .038493

$14.5514571 .086196-0.621374$

$63.221872-1.271464-0.172839$

$12.475865-2.041098-0.390150$

$14.076629-1.420839-0.839316$

$13.563085-1.3958700 .860624$

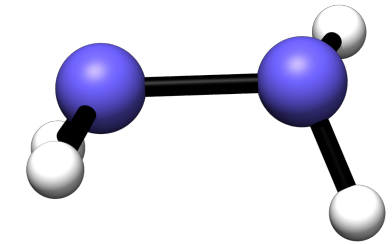

$5\left(\varphi=-70^{\circ}\right)\left(\mathrm{H}_{2} \mathrm{O}\right)$

01

$\#$ imaginary freq. $=0$

$E=-111.826142$
70.000000
$-0.720929$
$-0.090911$
10.606391
$-1.105573$
0.631759
$1-0.952062$
$-1.058207$
0.004620
$7-0.000000$
0.720929
$-0.090911$
$1-0.606391$
1.105573
10.952062
1.058207
0.631759
0.004620

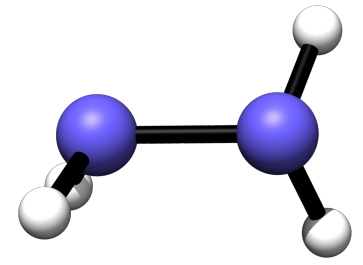

$5\left(\varphi=-31^{\circ}\right)\left(\mathrm{H}_{2} \mathrm{O}\right)$

01

$\#$ imaginary freq. $=0$

$\mathrm{E}=-111.830396$

$\begin{array}{llll}7 & 0.000000 & -0.717716 & -0.081868\end{array}$

$\begin{array}{llll}1 & -0.952541 & -1.018897 & -0.262227\end{array}$

$\begin{array}{llll}10.255042 & -1.079196 & 0.835302\end{array}$

$\begin{array}{llll}7 & 0.000000 & 0.717716 & -0.081868\end{array}$

$1-0.255042 \quad 1.079196 \quad 0.835302$

$\begin{array}{llll}1 & 0.952541 & 1.018897 & -0.262227\end{array}$

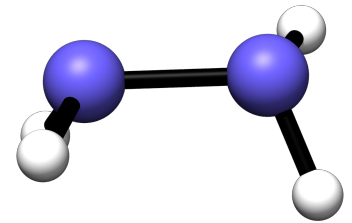

$5\left(\varphi=-70^{\circ}\right)($ Gas $)$

01

$\#$ imaginary freq. $=0$

$\mathrm{E}=-111.812069$

$\begin{array}{llll}7 & 0.000000 & 0.717716 & -0.103010\end{array}$

$\begin{array}{llll}1 & 0.958213 & 1.018897 & 0.044273\end{array}$

$\begin{array}{llll}1-0.546028 & 1.079196 & 0.676798\end{array}$

$1-0.000000-0.717716 \quad-0.103010$

$\begin{array}{llll}1 & 0.546028 & -1.079196 & 0.676798\end{array}$

$1-0.958213 \quad-1.018897 \quad 0.044273$

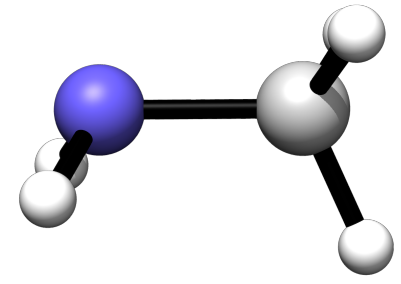

$6\left(\varphi=-70^{\circ}\right)\left(\mathrm{H}_{2} \mathrm{O}\right)$

01

$\#$ imaginary freq. $=0$

$E=-95.817704$ 


$\begin{array}{lrrr}6 & -0.706547 & -0.000327 & 0.016701 \\ 1-1.111945 & 0.934469 & -0.376086 \\ 1-1.053203 & -0.111650 & 1.051324 \\ 1-1.131350 & -0.816785 & -0.571017 \\ 70.752989 & -0.000168 & -0.130021 \\ 11.132545 & 0.808870 & 0.354910 \\ 11.132309 & -0.811760 & 0.350811\end{array}$

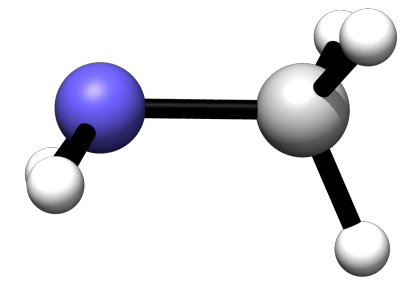

$6\left(\varphi=-57^{\circ}\right)\left(\mathrm{H}_{2} \mathrm{O}\right)$

01

$\#$ imaginary freq. $=0$

$E=-95.817790$

$6-0.706482-0.0000210 .016719$

$1-1.1213980 .881261-0.476761$

$1-1.0525390 .0000921 .057446$

$1-1.122411-0.880842-0.476839$

$70.752879-0.000059-0.129906$

11.1324570 .8103940 .352574

$11.132628-0.8103660 .352607$

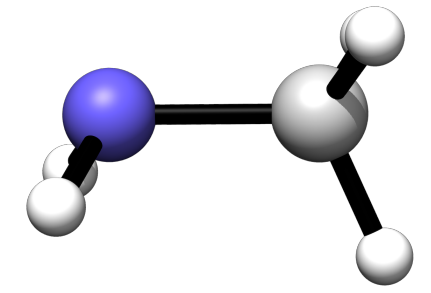

$6\left(\varphi=-70^{\circ}\right)($ Gas $)$

01

$\#$ imaginary freq. $=0$

$E=-95.810251$

$\begin{array}{rrrr}6 & -0.706547 & -0.000327 & 0.016701 \\ 1 & -1.111945 & 0.934469 & -0.376086 \\ 1-1.053203 & -0.111650 & 1.051324 \\ 1-1.131350 & -0.816785 & -0.571017 \\ 7 & 0.752989 & -0.000168 & -0.130021 \\ 11.132545 & 0.808870 & 0.354910 \\ 11.132309 & -0.811760 & 0.350811\end{array}$

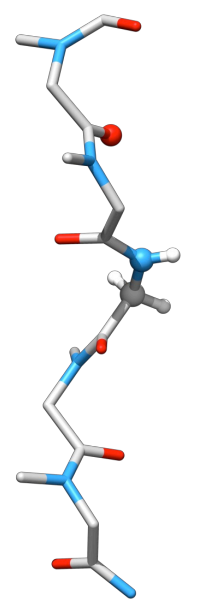

Gly Strand 1 Single Point

01

M06-2X(D3)/6-311+G(d,p)(Gas)

$E=-1366.887085$

wB97X-D/6-311+G(d,p)(Gas)

$E=-1367.009841$

M06-2X(D3)/6-311+G(d,p)( $\left.\mathrm{H}_{2} \mathrm{O}\right)$

$E=-1366.979826$

wB97X-D/6-311+G(d,p)( $\left.\mathrm{H}_{2} \mathrm{O}\right)$

$E=-1383.134249$

$67.4242360 .577497-0.513152$

$87.306776-0.329186-1.350262$

$18.0989391 .395273-0.719110$

76.7465790 .5751830 .628924

$65.737229-0.4480580 .901854$

$64.567476-0.370256-0.063909$

$84.3004850 .628358-0.695756$

66.8371681 .5548251 .719293

$16.173615-1.4467770 .886824$

16.2833792 .4592911 .467580

17.8796771 .7729451 .951021

$15.350038-0.2708091 .905232$

16.3796241 .1158082 .605869

$73.812176-1.465649-0.115813$

$62.550274-1.501874-0.856212$

$61.618690-0.321663-0.502418$

81.5493000 .0616670 .703172

$64.024448-2.7613430 .513371$

$12.688179-1.383611-1.930966$

$13.821621-2.7067091 .582939$

$15.046164-3.0986900 .339036$

$12.037660-2.442847-0.656470$

$13.339807-3.4817770 .065776$

$70.9414770 .234881-1.506968$

$6-0.1247641 .195424-1.282041$

$6-1.2270880 .580493-0.439299$

$8-1.352848-0.646818-0.389660$ 
$11.0870340 .066381-2.338260$ $10.2243821 .974050-0.820405$ $1-0.4997661 .476693-2.131205$ $7-2.0493031 .4171030 .223010$ $6-3.1363070 .8849631 .042173$ $6-4.2773630 .3647120 .162400$ $8-4.5493700 .939968-0.897025$ $6-1.9692652 .8879270 .280594$ $1-2.7807700 .0966461 .705687$ $1-1.9548103 .309585-0.724441$ $1-1.1006723 .2004420 .860232$ $1-3.5249721 .6964521 .657418$ $1-2.8619903 .2544610 .787342$ $7-4.934313-0.7008580 .600663$ $6-6.035462-1.281906-0.141622$ $6-7.279272-0.368201-0.128155$ $8-7.4692350 .4435950 .832509$ $6-4.690542-1.5117101 .785968$ $1-5.819934-1.366442-1.206751$ $1-4.620979-0.8775912 .669798$ $1-3.771850-2.0851701 .662481$ $1-6.286123-2.2606830 .267359$ $1-5.522059-2.2044681 .915458$ $7-8.106834-0.478706-1.161241$ $1-7.935364-0.927030-1.874825$ $1-8.996922-0.004755-1.104538$

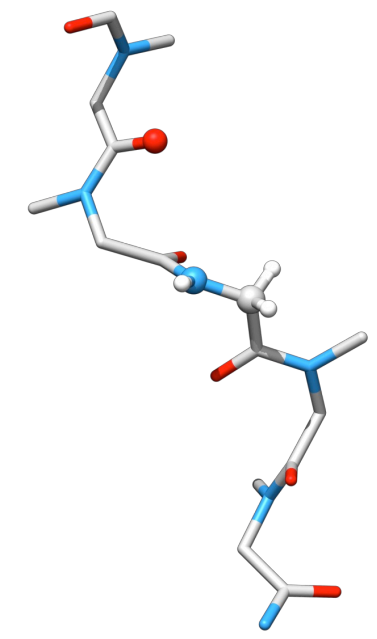

Gly Strand 2 Single Point 01

M06-2X(D3)/6-311+G(d,p)(Gas) $E=-1366.883366$ wB97X-D/6-311+G(d,p)(Gas) $E=-1367.005719$ M06-2X(D3)/6-311+G(d,p)( $\left.\mathrm{H}_{2} \mathrm{O}\right)$ $E=-1366.980311$ wB97X-D/6-311+G(d,p)( $\left.\mathrm{H}_{2} \mathrm{O}\right)$ $E=-1367.066921$
$67.3938390 .636650-0.301977$

$87.498540-0.283741-1.138700$ $17.9948841 .525421-0.425387$ 76.5634460 .5583940 .740639 $65.662322-0.5895040 .889994$ $64.490658-0.512654-0.094820$ $84.2395680 .507658-0.721895$ 66.4033761 .5692461 .796096 $16.199378-1.5295040 .763224$ 16.1086902 .5275651 .368447 17.3147221 .6532592 .388119 $15.254566-0.5681781 .900627$ 15.6036321 .2379592 .458486 $73.731062-1.580202-0.181080$ $62.492989-1.580464-0.958343$ $61.537873-0.435302-0.568591$ $81.427685-0.1013570 .629844$ $63.878881-2.9140530 .409978$ $12.669273-1.382969-2.015707$ $13.538436-2.9141481 .445447$ $14.920354-3.2311520 .356297$ $11.986007-2.538343-0.841991$ $13.269650-3.617018-0.158157$ $70.8331350 .143274-1.534890$ $6-0.1149641 .203580-1.245614$ $6-1.3072360 .818428-0.405492$ $8-1.526749-0.353746-0.083331$ $10.887906-0.063399-2.368156$ $10.3512301 .919204-0.784339$ $1-0.4484921 .560233-2.084042$ $7-2.1817701 .804233-0.112972$ $6-3.3492081 .5580030 .741629$ $6-4.2427020 .4676090 .153685$ $8-4.4645950 .418183-1.057956$ $6-1.9929023 .238120-0.402946$ $1-3.0493141 .2961381 .756317$ $1-2.2149623 .445633-1.449716$ $1-0.9820933 .549123-0.139051$ $1-3.9300012 .4791090 .790039$ $1-2.6934553 .8034120 .211685$ $7-4.791464-0.3939271 .000669$ $6-5.688260-1.4218160 .508768$ $6-6.941130-0.811448-0.170377$ $8-7.4718410 .2606620 .262683$ $6-4.515260-0.6478102 .417154$ $1-5.248157-2.017016-0.291323$ $1-5.0948500 .0254303 .048758$ $1-3.449935-0.5360582 .618882$ $1-5.992033-2.0719881 .329194$ $1-4.805030-1.6730342 .647478$ $7-7.405971-1.475851-1.210311$ 
$1-7.007695-2.140582-1.583285$

$1-8.292291-1.183299-1.596253$

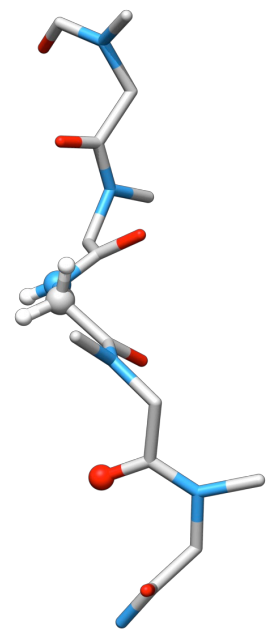

Gly Strand 3 Single Point

01

M06-2X(D3)/6-311+G(d,p)(Gas)

$E=-1366.882332$

wB97X-D/6-311+G(d,p)(Gas)

$E=-1367.004485$

M06-2X(D3)/6-311+G(d,p)( $\left.\mathrm{H}_{2} \mathrm{O}\right)$

$E=-1366.979740$

wB97X-D/6-311+G(d,p) $\left(\mathrm{H}_{2} \mathrm{O}\right)$

$E=-1367.065694$

$67.4919770 .429569-0.422316$

$87.515648-0.648628-1.016538$

$18.1921151 .200999-0.707100$

76.6209230 .6728780 .567828

$65.679740-0.3431430 .976630$

$64.518623-0.418416-0.026384$

$84.2624810 .487072-0.797071$

66.4749521 .9054391 .332686

$16.162845-1.3152861 .074859$

16.2424862 .7385570 .669381

17.3748832 .0979401 .916780

$15.274860-0.0627521 .949025$

15.6414671 .7795672 .023743

$73.788133-1.5189590 .006007$

$62.538860-1.657972-0.734121$

$61.608029-0.477739-0.431151$

81.6161620 .0767570 .697288

$64.021481-2.7519930 .765802$

$12.673091-1.592720-1.813854$

$13.760027-2.6138361 .814923$

$15.063396-3.0579350 .671401$

$12.050991-2.597226-0.473571$

$13.390219-3.5382920 .351865$

$70.832270-0.087624-1.428585$
$6-0.1187780 .999529-1.282348$

$6-1.2906020 .677347-0.353715$

$8-1.382126-0.4096110 .251155$

$10.838123-0.442116-2.212445$

$10.3395721 .778474-0.931172$

$1-0.4787401 .226643-2.154523$

$7-2.2010451 .650793-0.208544$

$6-3.3946831 .5314460 .617404$

$6-4.2824500 .3831320 .174529$

$8-4.3138570 .032198-1.021804$

$6-2.0783192 .990914-0.806221$

$1-3.1376681 .4256841 .671376$

$1-2.3099012 .953462-1.870677$

$1-1.0819393 .395589-0.628558$

$1-3.9667172 .4526870 .506986$

$1-2.8031183 .646262-0.323224$

$7-5.029384-0.1917701 .112913$

$6-5.951742-1.2484620 .782050$

$6-6.915513-0.820539-0.328162$

$8-7.4059400 .347660-0.333018$

$6-5.1170700 .0375132 .555722$

$1-5.456497-2.1150780 .344089$

$1-5.7549620 .8945062 .771956$

$1-4.1214610 .1917412 .971747$

$1-6.511799-1.5467731 .668304$

$1-5.554099-0.8460523 .020932$

$7-7.223631-1.742945-1.232474$

$1-6.884926-2.533208-1.251518$

$1-7.899073-1.500781-1.943272$

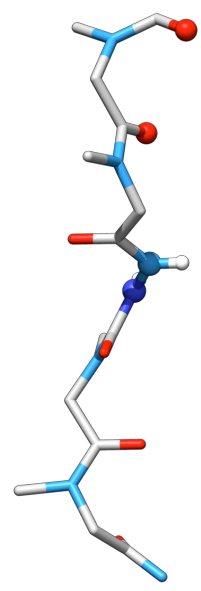

azGly Strand 1 Single Point

01

M06-2X(D3)/6-311+G(d,p)(Gas)

$E=-1382.924851$

wB97X-D/6-311+G(d,p)(Gas)

$E=-1383.042341$

M06-2X(D3)/6-311+G(d,p)( $\left.\mathrm{H}_{2} \mathrm{O}\right)$

$E=-1383.017485$ 
wB97X-D/6-311+G(d,p) $\left(\mathrm{H}_{2} \mathrm{O}\right)$

$E=-1383.134249$

$67.5244830 .341772-0.735760$

$87.193988-0.535296-1.548018$

76.9184340 .5104410 .441553

$65.795104-0.3205800 .877347$

$64.650589-0.288478-0.109874$

$84.4849120 .669737-0.839230$

67.3186031 .4480581 .508899

$16.118351-1.3505481 .028268$

16.9636962 .4533601 .281941

18.4001281 .4291591 .643233

$73.839481-1.339504-0.086106$

$62.642549-1.321057-0.905031$

$61.606539-0.388746-0.301525$

$81.556325-0.1306560 .885324$

$12.887202-0.939014-1.762198$

$6-1.3475710 .601623-0.127609$

$8-1.479754-0.5513580 .228455$

$7-0.2135241 .032868-0.826144$

$70.7443640 .144066-1.206946$

$1-0.1425551 .903994-1.074672$

$10.796793-0.125107-2.074825$

$7-2.2758001 .5419090 .074545$

$6-3.4861761 .2397710 .828027$

$6-4.3659400 .2087230 .167953$

$8-4.4142890 .146749-1.056226$

$6-2.1006142 .979793-0.157270$

$1-3.2398880 .9191151 .840263$

$1-2.3651303 .236631-1.183021$

$1-1.0825233 .2849020 .084597$

$7-5.121625-0.5487610 .957534$

$6-6.188387-1.3379110 .382266$

$6-7.099257-0.485849-0.503600$

$8-7.3261210 .697753-0.202706$

$6-5.078317-0.7037952 .407147$

$1-5.829144-2.106086-0.302535$

$1-5.5348330 .1517802 .904826$

$1-4.051174-0.8430422 .744323$

$7-7.679376-1.055136-1.549089$

$1-7.561723-1.876318-1.775846$

$12.229863-2.307424-1.116857$

$1-8.308361-0.552207-2.158630$

$18.2914351 .052355-0.963427$

15.4346640 .0664491 .830442

16.8538881 .1239302 .440071

$1-4.0039872 .0675490 .898017$

$1-2.7786453 .5130510 .509071$

$1-6.774366-1.7990781 .177284$

$1-5.647609-1.5948252 .671858$

$64.040148-2.5579650 .711380$
$14.988588-3.0197390 .436924$

$14.054291-2.3007731 .770509$

$13.226358-3.2568690 .518041$

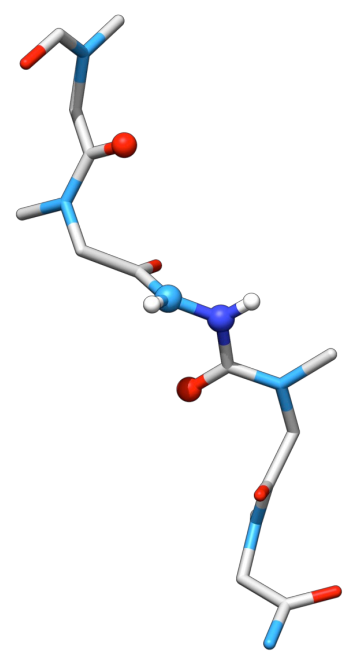

azGly Strand 2 Single Point

01

M06-2X(D3)/6-311+G(d,p)(Gas)

$E=-1382.924851$

wB97X-D/6-311+G(d,p)(Gas)

$E=-1383.042341$

M06-2X(D3)/6-311+G(d,p)( $\left.\mathrm{H}_{2} \mathrm{O}\right)$

$E=-1366.980311$

wB97X-D/6-311+G(d,p)( $\left.\mathrm{H}_{2} \mathrm{O}\right)$

$E=-1367.066921$

$67.3750600 .720698-0.611850$

$87.221016-0.276628-1.354798$

76.7100650 .8634950 .528311

$65.741407-0.1266240 .968336$

$64.636093-0.298133-0.055503$

$84.3126040 .600385-0.835468$

66.8265751 .9806041 .480704

$16.221143-1.0848591 .167678$

16.2456602 .8379841 .140796

17.8728502 .2383551 .644938

$73.997150-1.439914-0.018899$

$62.799217-1.671593-0.779864$

$61.755018-0.633406-0.370656$

$81.628738-0.2655170 .796606$

$13.003822-1.577621-1.846357$

$6-1.1661890 .231449-0.361768$

$8-1.240263-0.952206-0.023946$

$7-0.0879810 .705640-1.109466$

$70.957592-0.182496-1.385567$

$1-0.0386801 .578179-1.359038$

$11.033940-0.550675-2.213736$

$7-2.0898211 .137837-0.090380$

$\begin{array}{llll}6 & -3.150529 & 0.826790 & 0.879575\end{array}$ 
6 -4.349937 0.1585820 .232528

$8-4.4250500 .023820-0.984682$

$6-2.0302282 .571040-0.429566$

$1-2.7654870 .1936271 .678917$

$1-2.1332702 .712943-1.505366$

$1-1.1085603 .013241-0.051297$

$7-5.291470-0.2888791 .046668$

$6-6.444283-0.9860210 .527751$

$6-7.296071-0.117136-0.425013$

$8-7.3878871 .112359-0.278888$

$6-5.293658-0.3902212 .508909$

$1-6.156527-1.810326-0.124797$

$1-5.7168090 .5060852 .962428$

$1-4.284770-0.5703782 .880109$

$7-7.910410-0.774452-1.374049$

$1-7.807992-1.616228-1.517310$

$12.451916-2.682450-0.566203$

$1-8.556182-0.331906-2.012196$

$17.9947861 .550044-0.919301$

15.2924730 .2270811 .896480

16.4107181 .6517582 .433082

$1-3.4860881 .7640921 .323383$

$1-2.8684333 .0686540 .058176$

$1-7.059655-1.3549261 .348316$

$1-5.916082-1.2397202 .790050$

$64.419353-2.5943820 .787232$

$15.422728-2.8983320 .488981$

$14.421400-2.3197171 .842056$

$13.726994-3.4211290 .628402$

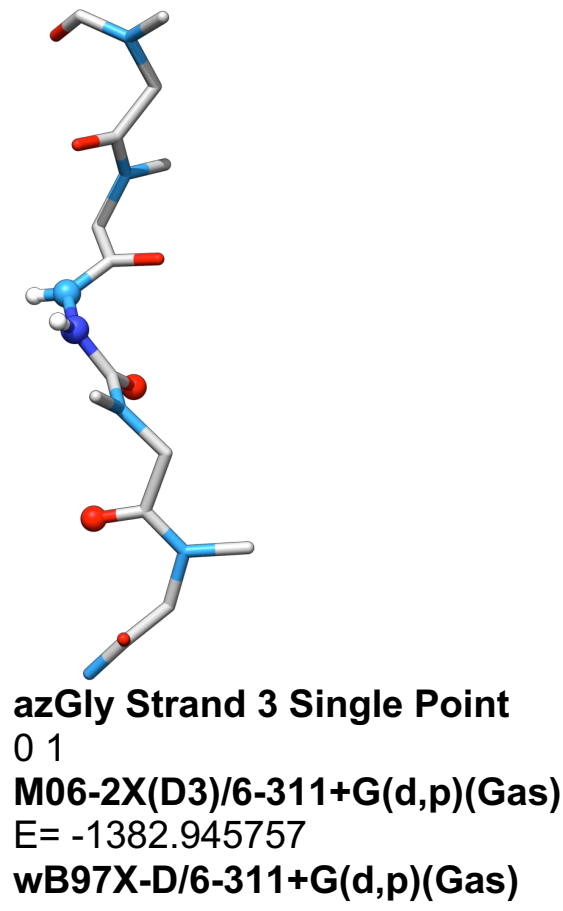

$E=-1383.065031$

M06-2X(D3)/6-311+G(d,p)( $\left.\mathrm{H}_{2} \mathrm{O}\right)$

$E=-1383.040586$

wB97X-D/6-311+G(d,p) $\left(\mathrm{H}_{2} \mathrm{O}\right)$

$E=-1383.127414$

$67.6118690 .634796-0.981816$

$87.515285-0.398802-1.654545$

76.9797730 .8040390 .181022

$66.096051-0.2290600 .730671$

$64.793381-0.315323-0.057131$

$84.4301970 .565168-0.837080$

67.0540731 .9995811 .037867

$16.590170-1.2000560 .764016$

16.8193962 .8953460 .462863

18.0347812 .0704461 .508282

$74.062164-1.4034160 .178787$

$62.780553-1.640105-0.469460$

$61.749043-0.643716-0.015367$

$81.626335-0.3159851 .166070$

$12.898486-1.543600-1.548756$

$6-1.2786640 .281223-0.097077$

$8-1.434787-0.8849030 .237375$

$7-0.1049060 .717859-0.729719$

$70.921771-0.189471-1.000228$

$1-0.0146011 .590778-0.967680$

$11.044547-0.497854-1.846946$

$7-2.1975431 .2228590 .114045$

$6-3.3909950 .9735930 .919540$

$6-4.4475500 .1834580 .180805$

$8-4.319457-0.110179-1.006420$

$6-2.0445392 .653576-0.188874$

$1-3.1262230 .4543811 .840635$

$1-2.1019982 .821677-1.264299$

$1-1.1085833 .0299430 .223961$

$7-5.511292-0.1746260 .896707$

$6-6.543838-1.0059850 .311211$

$6-7.383889-0.268061-0.728205$

$8-7.5556670 .963441-0.658355$

$6-5.717242-0.0717412 .357798$

$1-6.145679-1.851748-0.249372$

$1-6.2565500 .8403152 .613535$

$1-4.763219-0.1185702 .882913$

$7-7.913129-1.032257-1.666722$

$1-7.714310-1.857753-1.803206$

$12.455046-2.657049-0.250438$

$1-8.609862-0.654998-2.293091$

$18.1973661 .474369-1.326360$

15.8482210 .0541171 .753652

16.3075201 .8978511 .825528

$1-3.8209601 .9381591 .189434$

$1-2.8660123 .1914430 .284394$ 
$1-7.196537-1.3759181 .101929$ $1-6.320883-0.9220332 .675164$ $64.459206-2.4737601 .104866$ $15.398333-2.9141740 .769940$ $14.588323-2.0596662 .104843$ $13.685367-3.2410961 .126498$

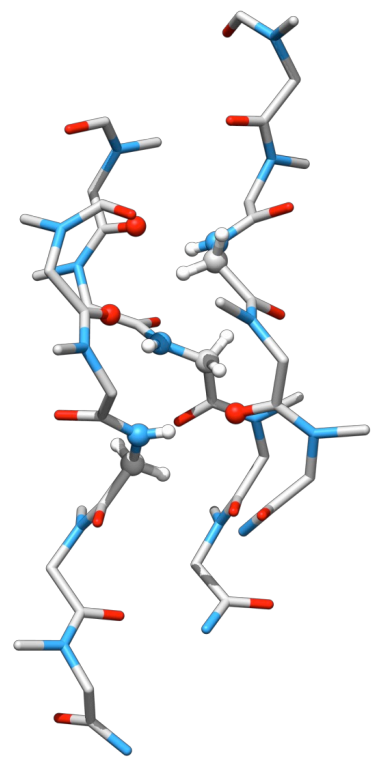

Gly Triple Helix Single Point 01

M06-2X(D3)/6-311+G(d,p)(Gas) $E=-4100.790200$

wB97X-D/6-311+G(d,p)(Gas)

$E=-4101.158144$

M06-2X(D3)/6-311+G(d,p)( $\left.\mathrm{H}_{2} \mathrm{O}\right)$

$E=-4101.001460$

wB97X-D/6-311+G(d,p) $\left(\mathrm{H}_{2} \mathrm{O}\right)$

$E=-4101.279623$

$66.8773532 .408436-2.158033$

$86.4633903 .508183-1.736873$

$17.7841251 .996928-1.739912$

$76.2361481 .724270-3.108560$

$64.9524572 .203108-3.632829$

$63.8161101 .965649-2.632385$

$83.9564611 .243344-1.654693$

$66.6680240 .447160-3.694994$

$15.0058113 .259016-3.897994$

$16.767967-0.315645-2.922828$

$17.5923000 .576724-4.258047$

$14.7271871 .639428-4.538157$

$15.8955260 .120053-4.390945$

$72.6634872 .527083-2.915823$

$61.4608532 .248293-2.132937$

$61.1577350 .741968-2.011879$
$81.357680-0.010578-2.988406$

$62.2900393 .472101-3.973102$

$11.5691092 .545556-1.089858$

$12.1186012 .947351-4.912967$

$13.0733824 .219829-4.097103$

$10.6069352 .761209-2.575477$

$11.3686703 .976087-3.681251$

$70.6582630 .298079-0.863564$

$60.311655-1.099672-0.681964$

$6-0.811464-1.623355-1.542209$

$8-1.476208-0.879557-2.270801$

$10.5108830 .790617-0.173832$

$11.097429-1.640122-0.862802$

$10.062733-1.2407570 .245148$

$7-1.124520-2.928218-1.395525$

$6-2.163194-3.559476-2.218052$

$6-3.509061-2.859731-2.039251$

$8-3.882818-2.484667-0.925974$

$6-0.369075-3.911323-0.596403$

$1-1.880520-3.574264-3.270657$

$1-0.610127-3.8055250 .461331$

$10.700807-3.804808-0.775589$

$1-2.273742-4.591535-1.885286$

$1-0.669029-4.909570-0.915198$

$7-4.265127-2.706491-3.118902$

$6-5.574486-2.093785-3.005167$

$6-6.512024-2.913636-2.081946$

$8-6.463170-4.184556-2.050512$

$6-3.948862-2.882608-4.538809$

$1-5.543318-1.121908-2.512640$

$1-4.091861-3.920692-4.838865$

$1-2.925044-2.566341-4.738487$

$1-6.023600-1.993428-3.993259$

$1-4.623972-2.257654-5.123411$

$7-7.348078-2.206875-1.346774$

$1-7.329993-1.350214-1.273256$

$1-8.059877-2.706933-0.833563$

64.1401133 .0856841 .915317

83.8915232 .4877722 .972356

15.0190172 .8087831 .352065

73.3598154 .0559341 .453464

62.0997364 .3878152 .118560

61.1045523 .2416342 .064256

81.1726742 .3484791 .248549

63.5725664 .8728380 .251708

12.2672174 .6939623 .151190

$13.2984374 .314601-0.643460$

14.6033205 .2244110 .206530

11.6596795 .2332101 .589640

12.9216985 .7447280 .317122

70.1068293 .3261152 .942019 
$6-1.0312122 .4064152 .911800$ $6-1.6665392 .2917791 .508542$ $8-1.7717833 .3357820 .798482$ $6-0.0889474 .2795304 .024805$ $1-0.7476041 .3769423 .130553$ $1-0.3904635 .2504503 .631738$ 10.8266354 .3756534 .608389 $1-1.7849632 .7360043 .626873$ $1-0.8790483 .9076784 .677159$ $7-2.0626911 .0786851 .122607$ $6-2.8686040 .870987-0.067970$ $6-4.1786001 .6303110 .036014$ $8-4.6164541 .9547761 .143973$ $1-1.8719390 .3526711 .543490$ $1-2.3876211 .182898-0.850680$ $1-3.061793-0.073519-0.174766$ $7-4.8308191 .912964-1.108244$ $6-6.1024302 .632312-1.071888$ $6-7.2314981 .719036-0.584239$ $8-7.2274450 .520477-0.885453$ $6-4.3871761 .623307-2.483861$ $1-6.0356903 .519991-0.442864$ $1-4.1530300 .564573-2.595028$ $1-3.5387502 .252987-2.751774$ $1-6.3392182 .952634-2.086494$ $1-5.2088901 .859814-3.159842$ $7-8.1824072 .2810320 .149947$ $6-9.3040591 .5207330 .664704$ $6-10.2686151 .091495-0.461125$

$8-10.3533621 .776181-1.529767$

$6-8.2875583 .6521120 .630504$ $1-8.9984300 .5635781 .087248$ $1-8.1594124 .352604-0.194717$ $1-7.5365973 .8361431 .398808$ $1-9.8393302 .1052601 .412977$ $1-9.2767673 .7979581 .064433$ $7-10.975261-0.012647-0.246980$ $1-10.843888-0.5536020 .408541$ $1-11.716468-0.231059-0.897371$ $610.283687-0.815863-0.523942$ $89.927650-0.784132-1.702245$ $111.078631-0.161321-0.198307$ $79.711638-1.6397230 .366193$ $68.653239-2.532947-0.042691$ $67.333104-1.758470-0.174318$ $87.155699-0.6770530 .353453$ $610.022682-1.7598251 .785469$ $18.896653-3.034261-0.979458$ $19.871586-0.8051572 .289333$ $111.039922-2.1272461 .920834$ $18.529422-3.2923630 .729357$
$19.337734-2.4861622 .222941$

$76.379696-2.343012-0.878012$ $65.012598-1.836584-0.926646$ $64.473832-1.6251620 .493291$ $84.839530-2.3685801 .439070$ $66.454127-3.567001-1.683259$ $14.941575-0.841537-1.365891$ $16.447394-4.450026-1.044258$ $17.351103-3.553365-2.302410$ $14.373905-2.532447-1.470658$ $15.582338-3.605111-2.336429$ $73.633312-0.6153710 .644001$ $63.021741-0.3093781 .924675$ $62.015130-1.3593342 .397601$ $81.785541-2.4010431 .751187$ $13.397626-0.0840640 .009727$ $13.715058-0.2308362 .597956$ 12.5616270 .5427911 .861861 $71.414434-1.1019423 .568282$ $60.415745-1.9691704 .177991$ $6-0.792919-2.1656083 .281648$ $8-1.144999-1.2700272 .488347$ 61.7362750 .0519704 .424499 $10.843488-2.9334624 .452360$ 11.2959970 .9613954 .015596 12.8157430 .1485234 .540801 $10.073142-1.4865225 .093291$ $11.302078-0.1234315 .408779$ $7-1.455096-3.3115783 .413047$ $6-2.652243-3.5673882 .652645$ $6-3.684590-2.4531352 .845628$ $8-3.869043-1.9448003 .991405$ $6-1.196619-4.4894204 .242405$ $1-2.478210-3.5446341 .576868$ $1-1.552477-4.3299915 .260269$ $1-0.131107-4.7191484 .245190$ $1-3.080415-4.5272802 .941397$ $1-1.732944-5.3374003 .816513$ $7-4.379663-2.0977551 .771324$ $1-4.252722-2.4213930 .984721$ $1-5.103768-1.4029681 .885530$ 


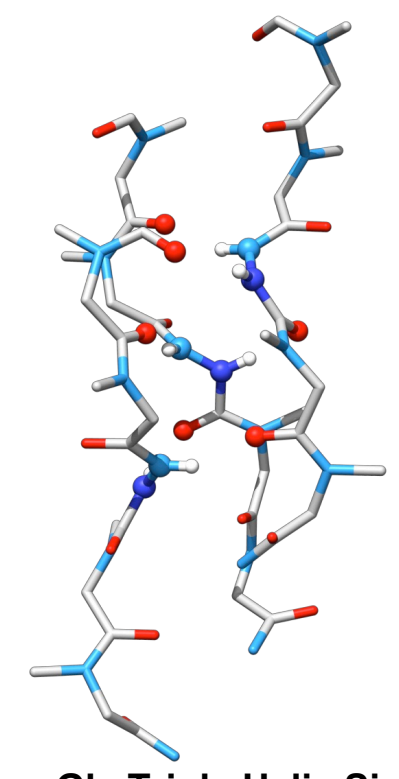

azGly Triple Helix Single Point

01

M06-2X(D3)/6-311+G(d,p)(Gas)

$E=-4148.992181$

wB97X-D/6-311+G(d,p)(Gas)

$E=-4149.345667$

M06-2X(D3)/6-311+G(d,p)( $\left.\mathrm{H}_{2} \mathrm{O}\right)$

$E=-4149.191179$

wB97X-D/6-311+G(d,p) $\left(\mathrm{H}_{2} \mathrm{O}\right)$

$E=-4149.464450$

$67.2300982 .899113-1.074077$

$86.6895343 .801246-0.422797$

$76.7401432 .446418-2.229668$

$65.4967782 .978788-2.796232$

$64.2834472 .505171-2.003428$

$84.3371641 .571294-1.203134$

$67.3320771 .384946-3.061484$

$15.5181684 .067114-2.852715$

$17.5089920 .488989-2.466453$

$18.2475751 .738794-3.535568$

$73.1519723 .160214-2.258316$

$61.8926052 .828133-1.608323$

$61.4008121 .472234-2.035735$

$81.4384511 .098674-3.209318$

$12.0361102 .815761-0.527882$

$6-0.920861-0.678400-1.918242$

$8-1.5684040 .295515-2.276381$

$70.323052-0.545911-1.282852$

$70.8501310 .723841-1.037075$

$10.783928-1.286793-1.027015$

$10.8224501 .072884-0.197723$
$7-1.336230-1.930743-2.104504$

$6-2.515407-2.244078-2.908648$

$6-3.813979-1.978172-2.181076$

$8-3.831927-1.632814-1.001017$

$6-0.575979-3.144734-1.772292$

$1-2.499530-1.681154-3.841903$

$1-0.559073-3.298081-0.693266$

$10.432214-3.084021-2.182123$

$7-4.924270-2.135135-2.898641$

$6-6.218542-1.824941-2.325271$

$6-6.657259-2.832959-1.266013$

$8-6.274525-4.017298-1.308938$

$6-5.058369-2.348671-4.356107$

$1-6.231487-0.878457-1.784809$

$1-5.144865-3.409841-4.589639$

$1-4.217969-1.901739-4.887219$

$7-7.470856-2.356191-0.340995$

$1-7.652502-1.523911-0.223031$

64.0966022 .2525582 .613119

83.6694011 .3392003 .335279

73.3999663 .3605392 .350657

62.0569253 .5934352 .883931

61.0993122 .4848032 .510482

81.2771071 .8206321 .508059

63.8749784 .5394291 .600278

12.0897173 .6977153 .968436

13.8158364 .3541920 .527763

14.8895574 .7975211 .903785

70.0559262 .3286963 .316764

$6-0.9778781 .3794142 .951062$

$6-1.8195511 .9305771 .813280$

$8-1.9620573 .1202861 .608280$

$1-0.4781360 .4925892 .561320$

$6-4.4288761 .8365840 .104353$

$8-4.8910152 .0806851 .200009$

$7-3.1401791 .312666-0.052992$

$7-2.3923640 .9763061 .033361$

$1-2.8214731 .145138-0.887272$

$1-2.2931650 .1016941 .264948$

$7-5.1115032 .003729-1.032614$

$6-6.4485342 .583497-1.016897$

$6-7.4613101 .744059-0.280437$

$8-7.3593980 .521655-0.291892$

$6-4.5536411 .910599-2.386149$

$1-6.4277493 .588469-0.595353$

$1-4.6060270 .884974-2.751456$ 
$1-3.5334242 .293800-2.406501$

$7-8.4807232 .3761250 .293644$ $6-9.6238211 .6182200 .752536$

$6-10.1662780 .703318-0.347075$

$8-10.1287281 .069928-1.533290$

$6-8.6717833 .8036070 .524038$

$1-9.3793830 .9205631 .553551$

$1-8.9626724 .311691-0.395383$

$1-7.7665274 .2464180 .939405$

$7-10.724880-0.4453430 .002521$

$1-10.786642-0.7221640 .814405$

$610.436403-0.918638-0.111324$

$89.993923-0.427067-1.175715$

$79.954559-2.0438880 .402698$

$68.862084-2.759860-0.234592$

$67.631959-1.881096-0.352684$

$87.410108-0.9473690 .421437$

$610.409960-2.7154541 .631588$

$19.154788-3.130772-1.216859$

$19.981613-2.2373222 .512502$

$111.498792-2.7432761 .673669$

$76.780917-2.214463-1.289619$

$65.479539-1.611321-1.391680$

$64.726872-1.855205-0.084143$

$84.817845-2.9185040 .528154$

$15.571489-0.538517-1.561199$

$62.067759-1.8825971 .402419$

$81.792494-2.4776790 .358026$

$73.124242-0.9733241 .461623$

$73.924823-0.8257070 .323414$

$13.321939-0.5345072 .232595$

$13.825385-0.095894-0.210191$

$71.388834-2.0128542 .529532$

$60.409164-3.1010672 .666288$

$6-0.969889-2.7051572 .171087$

$8-1.214508-1.5562831 .816611$

$61.701152-1.3624243 .814860$

$10.747638-3.9906762 .135129$

$11.502980-0.2920623 .758710$

$12.732150-1.5666824 .103689$

$7-1.880245-3.6633552 .119746$

$6-3.204824-3.3951191 .612003$

6 -3.966433 -2.337749 2.442773

$8-3.785675-2.2151963 .664963$

$6-1.730263-5.1082142 .315486$

$1-3.171849-2.9216100 .630778$
$1-1.903512-5.3819523 .356232$

$1-0.743270-5.4366561 .989802$

$7-4.810578-1.6037141 .765455$

$1-4.901017-1.6427450 .911115$

$11.1571833 .596435-1.847000$

$1-1.5914991 .0419663 .786345$

$14.959667-2.067104-2.234351$

$1-11.121080-1.081839-0.674234$

$1-7.936074-2.9860480 .296933$

$1-5.422457-0.9316632 .205969$

15.0487382 .1735142 .109543

$18.1215952 .406434-0.715051$

$111.172533-0.3921300 .477998$

$15.4016992 .594133-3.811663$

$16.6193231 .134129-3.847088$

$1-2.480545-3.305112-3.155813$

$1-1.078618-3.996937-2.229680$

$1-6.963774-1.793603-3.120097$

$1-5.970986-1.853680-4.688091$

11.6803324 .5258232 .463300

13.2238375 .3806261 .837984

$1-6.7819192 .667366-2.051267$

$1-5.1599752 .533734-3.043539$

$1-10.4072512 .2994061 .084683$

$1-9.4755503 .9271981 .249837$

$18.608411-3.6168420 .389371$

$110.051014-3.7442351 .602131$

$10.328287-3.3500303 .724388$

$11.046800-1.7871964 .576103$

$1-3.781415-4.3188361 .563130$

$1-2.476270-5.6137711 .702308$

$6-0.1623723 .0434424 .582616$

10.6687282 .8456395 .259556

$1-0.2262964 .1141914 .388938$

$1-1.0914142 .7004795 .037982$

$63.0465734 .276541-3.208896$

$13.6981895 .089423-2.888395$

$13.3476973 .938961-4.200593$

$12.0156784 .628994-3.242552$

$67.049163-3.223032-2.324864$

$17.927547-2.929160-2.899478$

$17.230080-4.188809-1.853012$

$16.188767-3.298978-2.989733$ 


\section{References}

${ }^{1}$ To be published.

${ }^{2}$ Berisio, R.; Vitagliano, L.; Mazzarella, L.; Zagari, A. Crystal structure of the collagen triple helix model [(Pro-ProGly)10]3. Protein Science. 2002, 11, 262-270.

${ }^{3}$ Okuyama, K.; Miyama, K.; Mizuno, K.; Bächinger, H. P. Crystal structure of (Gly-Pro-Hyp)9: Implications for the collagen molecular model. Biopolymers. 2012, 97, 607-616.

${ }^{4}$ Okuyama, K.; Hongo, C.; Fukushima, R.; Wu, G.; Narita, H.; Noguchi, K.; Tanaka, Y.; Nishino, N., Crystal structures of collagen model peptides with Pro-Hyp-Gly repeating sequence at $1.26 \AA$ resolution: Implications for proline ring puckering. Biopolymers. 2004, 76, 367-377.

${ }^{5}$ Schumacher, M.; Mizuno, K.; Bächinger, H. P. The Crystal Structure of the Collagen-like Polypeptide (Glycyl-4(R)hydroxyprolyl-4(R)-hydroxyprolyl) $)_{9}$ at $1.55 \AA$ Resolution Shows Up-puckering of the Proline Ring in the Xaa Position. J. Biol. Chem. 2005, 280, 20397-20403.

${ }^{6}$ Widmer, C.; Gebauer, J. M.; Brunstein, E.; Rosenbaum, S.; Zaucke, F.; Drögemüller, C.; Leeb, T.; Baumann, U., Molecular basis for the action of the collagen-specific chaperone Hsp47/SERPINH1 and its structure-specific client recognition. Proc. Natl. Acad. Sci. U. S. A. 2012, 109, 13243-13247.

${ }^{7}$ Okuyama, K.; Hongo, C.; Wu, G.; Noguchi, K.; Ebisuzaki, S.; Tanaka, Y.; Nishino, N.; Bächinger, H. P. Highresolution structures of collagen-like peptides [(Pro-Pro-Gly)4-Xaa-Yaa-Gly-(Pro-Pro-Gly)4]: Implications for triplehelix hydration and Hyp(X) puckering. Biopolymers. 2009, 91, 361-372.

${ }^{8}$ Jiravanichanun, N.; Hongo, C.; Wu, G.; Noguchi, K.; Okuyama, K.; Nishino, N.; Silva, T. Unexpected Puckering of Hydroxyproline in the Guest Triplets, Hyp-Pro-Gly and Pro-alloHyp-Gly Sandwiched between Pro-Pro-Gly Sequence. ChemBioChem. 2005, 6, 1184-1187.

${ }^{9}$ Okuyama, K.; Kawaguchi, T.; Shimura, M.; Noguchi, K.; Mizuno, K.; Bächinger, H. P. Crystal structure of the collagen model peptide (Pro-Pro-Gly)4-Hyp-Asp-Gly-(Pro-Pro-Gly)4 at $1.0 \AA ̊$ resolution. Biopolymers. 2013, 99, 436-447.

${ }^{10}$ Okuyama, K.; Morimoto, T.; Narita, H.; Kawaguchi, T.; Mizuno, K.; Bächinger, H. P.; Wu, G.; Noguchi, K., Two crystal modifications of (Pro-Pro-Gly)4-Hyp-Hyp-Gly-(Pro-Pro-Gly)4 reveal the puckering preference of Hyp(X) in the $\operatorname{Hyp}(\mathrm{X}): \operatorname{Hyp}(\mathrm{Y})$ and $\operatorname{Hyp}(\mathrm{X}): \operatorname{Pro}(\mathrm{Y})$ stacking pairs in collagen helices. Acta Crystallogr. Sect. D. Struct. Biol. 2010, 66, 88-96.

${ }^{11}$ Okuyama, K.; Miyama, K.; Morimoto, T.; Masakiyo, K.; Mizuno, K.; Bächinger, H. P., Stabilization of triplehelical structures of collagen peptides containing a Hyp-Thr-Gly, Hyp-Val-Gly, or Hyp-Ser-Gly sequence. Biopolymers. 2011, 95, 628-640.

${ }^{12}$ Okuyama, K.; Haga, M.; Noguchi, K.; Tanaka, T., Preferred Side-Chain Conformation of Arginine Residues in a Triple-Helical Structure. Biopolymers. 2014, 101, 1000-1009.

${ }^{13}$ Kasznel, A. J.; Zhang, Y.; Hai, Y.; Chenoweth, D. M. Structural Basis for Aza-Glycine Stabilization of Collagen. J. Am. Chem. Soc. 2017, 139, 9427-9430.

${ }^{14}$ Kasznel, A. J.; Harris, T.; Porter, N. J.; Zhang, Y.; Chenoweth, D. M. Aza-Proline Effectively Mimics L-Proline Stereochemistry in Triple Helical Collagen. Chem. Sci. 2019, 10, 6979-6983.

${ }^{15}$ Emsley, J.; Knight, C. G.; Farndale, R. W.; Barnes, M. J. Structure of the integrin alpha2beta1-binding collagen peptide. J. Mol. Biol. 2004, 335, 1019-1028.

${ }^{16}$ Plonska-Brzezinska, M. E.; Bobrowska, D. M.; Sharma, A.; Rodziewicz, P.; Tomczyk, M.; Czyrkoa, J.; Brzezinski, K., Triple helical collagen-like peptide interactions with selected polyphenolic compounds. RSC Adv. 2015, 5, 9544395453.

${ }^{17}$ Kramer, R. Z.; Venugopal, M. G.; Bella, J.; Mayville, P.; Brodsky, B.; Berman, H. M. Staggered molecular packing in crystals of a collagen-like peptide with a single charged pair. J. Mol. Biol. 2000, 301, 1191-1205.

${ }^{18}$ Okuyama, K.; Narita, H.; Kawaguchi, T.; Noguchi, K.; Tanaka, Y.; Nishino, N., Unique Side Chain Conformation of a Leu Residue in a Triple-Helical Structure. Biopolymers. 2007, 86, 212-221.

${ }^{19}$ Motooka, D.; Kawahara, K.; Nakamura, S.; Doi, M.; Nishi, Y.; Nishiuchi, Y.; Kee Kang, Y.; Nakazawa, T.; Uchiyama, S.; Yoshida, T.; Ohkubo, T.; Kobayashi, Y. The triple helical structure and stability of collagen model peptide with 4(S)-hydroxyprolyl-pro-gly units. Biopolymers. 2011, 98, 111-121.

${ }^{20}$ Kramer, R. Z.; Bella, J.; Mayville, P.; Brodsky, B.; Berman, H. M. Sequence dependent conformational variations of collagen triple-helical structure. Nat. Struct. Mol. Biol. 1999, 6, 454-457. 